
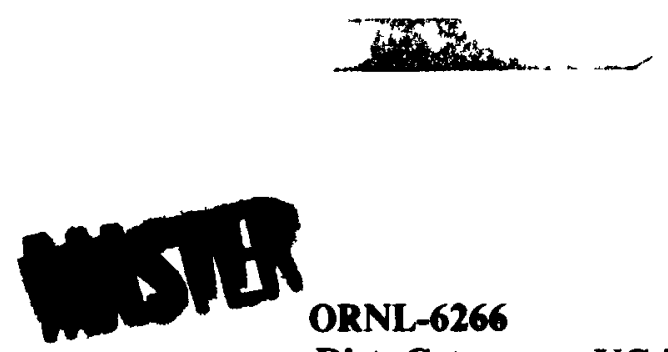

Dist. Category - UC-79d

Engineering Physics and Mathematics Division

\title{
FISSION-PRODUCT YIELD DATA FROM THE US/UK JOINT EXPERIMENT IN THE DOUNREAY PROTOTYPE FAST REACTOR
}

\section{J. K. Dickens \\ S. Raman*}

Date Published: April 1986

*Physics Division

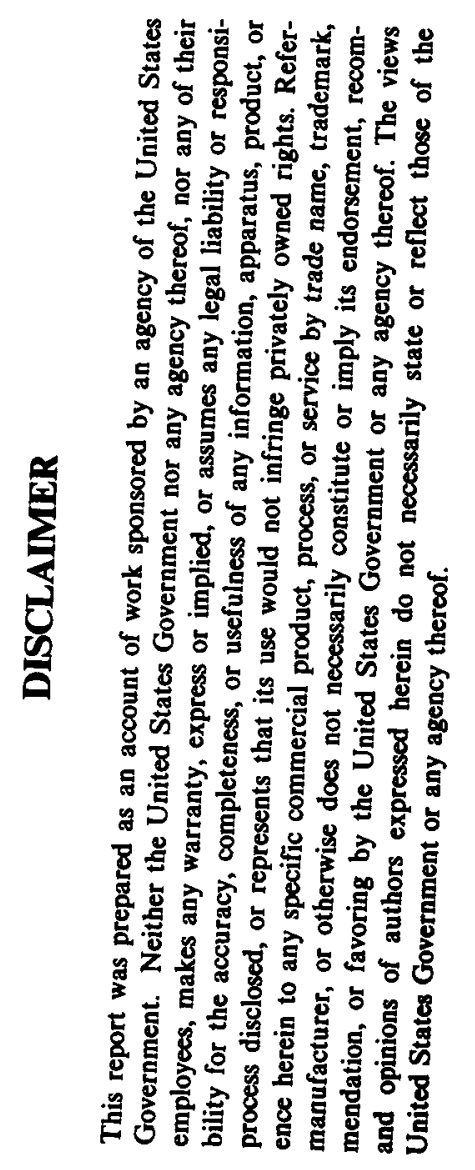

\author{
Prepared by the \\ Oak Ridge National Laboratory \\ Oak Ridge, Tennessee 37831 \\ operated by \\ Martin Marietta Energy Systems, Inc. \\ for the \\ U.S. DEPARTMENT OF ENERGY \\ under Contract No. DE-AC05-840R21400
}




\section{DISCLAIMER}

This report was prepared as an account of work sponsored by an agency of the United States Government. Neither the United States Government nor any agency Thereof, nor any of their employees, makes any warranty, express or implied, or assumes any legal liability or responsibility for the accuracy, completeness, or usefulness of any information, apparatus, product, or process disclosed, or represents that its use would not infringe privately owned rights. Reference herein to any specific commercial product, process, or service by trade name, trademark, manufacturer, or otherwise does not necessarily constitute or imply its endorsement, recommendation, or favoring by the United States Government or any agency thereof. The views and opinions of authors expressed herein do not necessarily state or reflect those of the United States Government or any agency thereof. 


\section{DISCLAIMER}

Portions of this document may be illegible in electronic image products. Images are produced from the best available original document. 
Printed in the United States of America. Available from National Technical Information Service

U.S. Department of Commerce

5285 Port Royal Road, Springfield, Virginia 22161

NTIS price codes-Printed Copy: A04; Microfiche A01

This report was prepared as an account of work sponsored by an agency of the United States Government. Neither the United States Government nor any agency thereof, nor any of their employees, makes any warranty, express or implied, or assumes any legal liability or responsibility for the accuracy, completeness, or usefulness of any information, apparatus, product, or process disclosed, or represents that its use would not infringe privately owned rights. Reference herein to any specific commercial product, process, or service by trade name, trademark, manufacturer, or otherwise, does not necessarily constitute or imply its endorsement, recommendation, or favoring by the United States Government or any agency thereof. The views and opinions of authors expressed herein do not necessarily state or reflect those of the United States Government or any agency thereof. 
TABLE OF CONTENTS

\section{LIST OF TABLES}

LIST OF FIGURES

\section{ABSTRACT}

1. INTRODUCTION

2. EXPERIMENTAL DETAILS

2.1. Gamma-Ray-Assay Sample Configuration

2.2. Gamma-Ray-Assay Counting Configuration

2.3. Gamma-Ray-Assay Counting Measurements

2.4. Gamma-Ray-Assay Counting Analyses

vii

ix

1

3

3

5

3. RESULTS AND ANALYSES

3.1. Analysis of ${ }^{137} \mathrm{Cs}$ Absolute Yields

3.1.1. ${ }^{230}$ Th Sample

3.1.2. ${ }^{231} \mathrm{~Pa}$ Sample

3.1.3. ${ }^{238} \mathrm{Pu}$ Sample

3.1.4. ${ }^{239} \mathrm{Pu}$ Sample

3.1.5. ${ }^{240} \mathrm{Pu}$ Sample

3.1.6. ${ }^{241} \mathrm{Pu}$ Sample

3.1.7. ${ }^{241}$ Am Sample

3.1.8. ${ }^{243}$ Am Sample

3.1.9. ${ }^{243} \mathrm{Cm}$ Sample

3.1.10. ${ }^{244} \mathrm{Cm}$ Sample

3.1.11. Other Samples

3.3. Comparisons of Experimental and Evaluated Fission-Product Yields 31

3.3.1. ${ }^{91} \mathrm{Y}$ Yield

3.3.2. ${ }^{95} \mathrm{Zr}$ Yield

3.3.3. ${ }^{103} \mathrm{Ru}$ Yield

3.3.4. ${ }^{106} \mathrm{Ru}-{ }^{106} \mathrm{Rh}$ Yield

3.3.5. ${ }^{125} \mathrm{Sb}$ Yield

3.3.6. ${ }^{141} \mathrm{Ce}$ Yield

3.3.7. ${ }^{144} \mathrm{Ce}-{ }^{144} \mathrm{Pr}$ Yield

46

46

3.3.8. ${ }^{155} \mathrm{Eu}$ Yield

46

3.3.9. Summary

3.4.8. ${ }^{239} \mathrm{Pu}$ Sample 
3.4.10. ${ }^{241} \mathrm{Pu}$ Sample

3.4.11. ${ }^{244} \mathrm{Pu}$ Sample

3.4.12. ${ }^{241}$ Am Sample

3.4.13. ${ }^{243} \mathrm{Am}$ Sample

3.4.14. ${ }^{244} \mathrm{Cm}$ Sample

3.4.15. ${ }^{246} \mathrm{Cm}$ Sample

54

3.4.16. ${ }^{248} \mathrm{Cm}$ Sample

55

3.4.17. Summary

55

4. SUMMARY AND CONCLUSIONS

5. ACKNOWLEDGEMENTS 


\section{LIST OF TABLES}

Table 1. SSMS Analysis of High Purity Vanadium Used for UK/US Physics Samples

Table 2. Sample Principal Actinide Masses $\quad 4$

$\begin{array}{ll}\text { Table 3. Nuclear Data for Fission Products } & 17\end{array}$

Table 4. Absolute Experimental Yields of Fission-Product ${ }^{137} \mathrm{Cs}$ in Numbers of Atoms as of the End of the PFR Irradiation $\begin{array}{ll}\text { (September 1, 1983) } & 19\end{array}$

Table 5. Background Radionuclide Decay Observed 20

Table 6. SSMS Selected Impurity Analyses of Actinide Samples 21

Table 7. Fission-Product Yields for Fast-Neutron Fission 32

Table 8. $\quad{ }^{95} \mathrm{Nb}$ Fission-Product Yields for Fast-Neutron Fission 34

Table 9. Irradiation History at the Dounreay PFR for the Present Experiment $\quad 35$

Table 10. Adjustment factors for the experimental ratio data 35 
, 


$$
\text { vii /vi" }
$$

\section{LIST OF FIGURES}

Figure 1. Schematic representation of the sample orientation with respect to the $\mathrm{Ge}(\mathrm{Li})$ (or intrinsic $\mathrm{Ge}$ ) detector.

Figure 2. Portion of the gamma-ray and $x$-ray spectrum obtained from a measurement of the ${ }^{234} \mathrm{U}$ sample using the intrinsic-Ge low-energy photon detector.

Figure 3. Somewhat higher-energy portion of the spectrum shown in Fig. 2.

Figure 4. Higher-energy portion of the spectrum of Figs. 2 and $3 . \quad 10$

Figure 5. Highest-energy portion of the spectrum of Figs. 2 to 4.

Figure 6. Portion of the gamma-ray spectrum from a measurement of the ${ }^{238} \mathrm{U}$ sample using the large-volume $\mathrm{Ge}(\mathrm{Li})$ photon detector.

Figure 7. Higher-energy portion of the spectrum of Fig. $6 . \quad 13$

Figure 8. Higher-energy portion of the spectrum of Figs. 6 and $7 . \quad 14$

Figure 9. Highest-energy portion of the spectrum of Figs. 6 to 8.

Figure 10. Absolute yields of ${ }^{137} \mathrm{Cs}$ following fast-neutron fission of 19 different actinides between ${ }^{230} \mathrm{Th}$ and ${ }^{248} \mathrm{Cm}$.

Figure 11. Relative yields of ${ }^{91} \mathrm{Y}$ following fast-neutron fission of actinides between ${ }^{230} \mathrm{Th}$ and ${ }^{248} \mathrm{Cm}$.

Figure 12. Relative yields of ${ }^{95} \mathrm{Zr}$ following fast-neutron fission of actinides between ${ }^{230} \mathrm{Th}$ and ${ }^{248} \mathrm{Cm}$.

Figure 13. Relative yields of ${ }^{103} \mathrm{Ru}$ following fast-neutron fission of actinides between ${ }^{230} \mathrm{Th}$ and ${ }^{248} \mathrm{Cm}$.

Figure 14. Relative yields of ${ }^{106} \mathrm{Ru}-{ }^{106} \mathrm{Rh}$ following fast-neutron fission of actinides between ${ }^{230} \mathrm{Th}$ and ${ }^{248} \mathrm{Cm}$.

Figure 15. Relative yields of ${ }^{125} \mathrm{Sb}$ following fast-neutron fission of Actinides Between ${ }^{230} \mathrm{Th}$ and ${ }^{248} \mathrm{Cm}$

Figure 16. Relative yields of ${ }^{141} \mathrm{Ce}$ following fast-neutron fission of actinides between ${ }^{230} \mathrm{Th}$ and ${ }^{248} \mathrm{Cm}$.

Figure 17. Relative yields of ${ }^{144} \mathrm{Ce}-{ }^{144} \mathrm{Pr}$ following fast-neutron fission of actinides between ${ }^{230} \mathrm{Th}$ and ${ }^{248} \mathrm{Cm}$.

Figure 18. Relative yields of ${ }^{155} \mathrm{Eu}$ following fast-neutron fission of actinides between ${ }^{230} \mathrm{Th}$ and ${ }^{248} \mathrm{Cm}$.

Figure 19. Portion of the gamma-ray spectrum from a measurement of the ${ }^{239} \mathrm{Pu}$ sample using the intrinsic-Ge high-resolution detector. 
'

, 


\begin{abstract}
The United States and the United Kingdom have been engaged in a joint research program in which samples of fissile and fertile actinides have been incorporated in fuel pins and irradiated in the Dounreay Prototype Fast Reactor in Scotland. The purpose of this portion of the program is to study both the materials behavior and the nuclear physics results primarily measurements of the fission-product yields in the irradiated samples and secondarily information on the amounts of heavy elements in the samples. In the measurements highresolution detectors were used to observe (and quantitatively measure) the gamma rays and $x$ rays corresponding to the decay of several long-lived radioisotopes. Two series of measurements were made, one nine months following the end of the irradiation period and another approximately six months later. The samples were milligram quantities of actinide oxides of ${ }^{248} \mathrm{Cm},{ }^{246} \mathrm{Cm},{ }^{244} \mathrm{Cm},{ }^{243} \mathrm{Cm},{ }^{243} \mathrm{Am},{ }^{241} \mathrm{Am},{ }^{244} \mathrm{Pu},{ }^{241} \mathrm{Pu},{ }^{240} \mathrm{Pu},{ }^{239} \mathrm{Pu},{ }^{238} \mathrm{Pu},{ }^{238} \mathrm{U}$, ${ }^{236} \mathrm{U},{ }^{235} \mathrm{U},{ }^{234} \mathrm{U},{ }^{233} \mathrm{U},{ }^{231} \mathrm{~Pa},{ }^{232} \mathrm{Th}$, and ${ }^{230} \mathrm{Th}$ that had been encapulated in vanadium holders and exposed in the core to a total fluence of approximately $2.7 \times 10^{22}$ "fast" neutrons over a period of about 12 months. The fission products identified were ${ }^{91} \mathrm{Y},{ }^{95} \mathrm{Zr},{ }^{95} \mathrm{Nb},{ }^{103} \mathrm{Ru},{ }^{106} \mathrm{Rh}$ (following decay of ${ }^{106} \mathrm{Ru}$ ), ${ }^{110 \mathrm{~m}} \mathrm{Ag},{ }^{125} \mathrm{Sb},{ }^{134} \mathrm{Cs},{ }^{137} \mathrm{Cs},{ }^{141} \mathrm{Ce},{ }^{144} \mathrm{Ce},{ }^{144} \mathrm{Pr}$, and ${ }^{155} \mathrm{Eu}$. Because of uncertainties associated with the experiment (e.g. initial sample compositions, effective fission cross sections, etc.), not all the fission-product yields could be obtained on an absolute basis. Therefore, the absolute yields of the fission product ${ }^{137} \mathrm{Cs}$ in the various samples were designated as monitor data for determining the yields of the other fission products. The resulting relative-yield fission-product data were manipulated for comparison with presently existing evaluated data; the comparisons are generally favorable and the exceptions are discussed. In addition, determinations of the heavy element contents of the samples provided information on both the initial sample composition and the actinides created during the irradiation.
\end{abstract}




\section{INTRODUCTION}

Following discussions that had been formally initiated as early as 1977, an agreement for an experimental program entitled "Higher Actinides Agreement" was signed in May 1979 as part of a long-term cooperative program in the field of Liquid Metal Fast Breeder Reactor (LMFBR) technology between the United Kingdom (UK) and the United States under the LMFBR agreement of 1976. As originally conceived, the experiment was aimed primarily at an evaluation of materials behavior of the higher actinides in a fuel-pin type of irradiation. However, the final agreement called for an additional experiment to carry out simultaneous irradiations of "physics specimens" of fissile and fertile fuels in order to improve our knowledge of basic nuclear physics phenomena, e.g., cross-sections and fission-product yields. The present report details the aspects of the experiment primarily concerned with deducing fission-product yields.

In an overall picture the experiment followed a rather straight-forward chronology. Complete details have been given in a series of reports ${ }^{1-3}$ of work sponsored by the U.S. Department of Energy, Office of Breeder Technology Projects, from which experimental aspects important to the present experiment have been taken. The sample preparation was performed mostly during 1980 at the Oak Ridge National Laboratory (ORNL) and was reported in detail by Quinby et al. ${ }^{1}$ The assembly of the fuel pins was the responsibility of Hanford Engineering Development Laboratory (HEDL), which also shipped the pins to the UK for irradiation. The fuel pins were inserted into the Dounreay Prototype Fast Reactor (PFR) in early 1982, and irradiation began on August 24, 1982. It was anticipated that a total-neutron fluence equivalent to 90 Full Power Days (FPD) would be obtained; however, at the end of the irradiations one year later on August 31, 1983, PFR operations reported a total irradiation power history equivalent to $63 \mathrm{FPD}$. Following the removal of the fuel pins, the fuel pin containing the physics samples was returned to the U.S., ultimately arriving at ORNL during May 1984. Individual samples were removed from the fuel pins, and samples suitable for gamma-ray assay were prepared from predetermined aliquots of the total samples. Chemical separations of the actinides or of selected fission elements were not performed; each gamma-ray-assay sample was to reflect a specific amount of the total unseparated sample. One consequence of this choice of sample preparation was that overall sample sizes were limited for several actinides because of the large specific activity associated with the actinide contents of the irradiated sample. A compensation to this limitation, however, was the ability to determine principal sample actinide masses from the gamma-ray assay for some of the actinides in the sample as well as for the available fission products in the sample.

The predetermined aliquots for the gamma-ray-assay samples were determined after a study of the preanalysis calculations of Broadhead et al., ${ }^{2}$ which were based upon an irradiation assumed equivalent to $90 \mathrm{FPD}$. To a considerable extent these calculations relied on the details of the physics specimens as given in the report of Walker et al. ${ }^{3}$ As it happened, the fast neutrons from the actual irradiation history produced less activity in the samples than had been estimated; however, the differences were readily compensated for during the actual gamma-ray-counting experiments. 
Some of the details of the physics samples encapsulation are repeated from Quinby et al. ${ }^{1}$ Physics specimens were required to be encapsulated in a high-purity material that would not produce an undesirable background after irradiation. Capsules made from high-purity vanadium were used for this purpose. A spark source mass spectrographic (SSMS) analysis of the vanadium is shown in Table 1 .

Table 1. SSMS analysis of high-purity vanadium used for UK/US physics samples ${ }^{a}$

\begin{tabular}{lc}
\hline Element & Mass (ppm) \\
\hline & \\
$\mathrm{Ag}$ & 5 \\
$\mathrm{As}$ & 2 \\
$\mathrm{~B}$ & 5 \\
$\mathrm{Ca}$ & 0.3 \\
$\mathrm{Fe}$ & 100 \\
$\mathrm{~K}$ & 1 \\
$\mathrm{Mg}$ & 10 \\
$\mathrm{Mo}$ & 50 \\
$\mathrm{Na}$ & 2 \\
$\mathrm{Nb}$ & 10 \\
$\mathrm{Ni}$ & 20 \\
$\mathrm{P}$ & 20 \\
$\mathrm{Si}$ & 300 \\
$\mathrm{Ta}$ & 100 \\
$\mathrm{Ti}$ & 20 \\
$\mathrm{~V}$ & Major \\
$\mathrm{W}$ & 40 \\
$\mathrm{Zr}$ & 10 \\
$\mathrm{~S}$ & 100 \\
$\mathrm{~F}$ & 0.5 \\
\hline${ }^{\mathrm{Elements}}$ &
\end{tabular}


The vanadium capsules were quite small, having a $7.6-\mathrm{mm}$ length, a $1.5-\mathrm{mm}$ outside diameter, and an interior volume of $0.52 \mathrm{~mm}^{3}$. We remark on both the SSMS impurity analysis and the small size of the capsules because these aspects impacted the overall results of the gamma-ray-assay experiment. The small size and the hard constituency of the vanadium presented a serious challenge in extracting the irradiated actinide samples from the capsules, impacting upon the preparation of at least five of the gamma-ray-assay samples. In addition, peaks were observed in the raw data which could be ascribed to the detection of gamma rays following the decay of radioisotopes created by neutron interactions with capsule impurity elements. Knowledge of these impurities was particularly important for those cases in which a gamma ray from decay of an impurity radionuclide was accidentally degenerate (to within the resolving power of the measuring system) with a gamma ray from decay of a desired radionuclide.

The remainder of this report is dedicated to a complete discussion of the gamma-ray-assay measurements and data reduction. Some detail is presented to provide the reader with a sufficient description of the experiment so as to judge the quality of the results, and, in addition, to present some problems which were encountered during the experiment and our solutions to and/or recommendations concerning such problems.

\section{EXPERIMENTAL DETAILS}

\subsection{GAMMA-RAY-ASSAY SAMPLE CONFIGURATION}

The fuel pin containing the physics samples was opened about nine months following the .nd of the irradiation. This process took place in a well-shielded hood using remote manipulators. Each vanadium capsule was sawed open and its solid oxide sample was removed and then dissolved in acid $\left(\mathrm{HNO}_{3}\right)$. A portion of this solution was determined by aliquot for the present measurements, the amount being deduced a priori upon the expected activity of the total sample indicated by preanalysis calculations of Broadhead et al. ${ }^{2}$ Each aliquot was diluted with $\mathrm{HNO}_{3}$ to a 4-ml volume and transferred to a glass bottle. The material was sealed in the bottle with a semi-permanent sealing plastic cap impervious to the acid solution.

The given initial sample masses and our aliquots (shares) are given in Table 2, along with the half lives of the principal isotopes taken from the Table of Isotopes. ${ }^{4}$ (For three isotopes, namely ${ }^{240} \mathrm{Pu},{ }^{241} \mathrm{Am}$, and ${ }^{244} \mathrm{Cm}$, there were two separate samples.) The initial masses were obtained from the report of Quinby et al. ${ }^{1}$ For several of the samples, the total masses were computed, or recomputed, as of August 24, 1982, the date of the beginning of the irradiation, with supplemental information about the sample material (e.g., isotopic \%, concentrations, dates of measurements) obtained from data given in the report by Walker et al. ${ }^{3}$ Since the gamma-ray-assay samples contained the principal actinides being studied, the gamma-ray measurements themselves provided an independent determination of the amount of the principal actinide. 
Table 2. Sample principal actinide masses

\begin{tabular}{|c|c|c|c|c|}
\hline $\begin{array}{c}\text { Principal } \\
\text { isotope }\end{array}$ & $\begin{array}{c}\text { Sample } \\
\text { No. }\end{array}$ & $\begin{array}{c}\text { Total } \\
\text { mass }(\mathrm{mg})^{a}\end{array}$ & $\begin{array}{l}\gamma \text {-ray assay } \\
\text { aliquot }(\%)\end{array}$ & $\begin{array}{c}\text { Isotope half } \\
\text { life }(\mathrm{yr})^{b}\end{array}$ \\
\hline${ }^{230} \mathrm{Th}$ & 31 & 2.921 & 10.0 & $8.0 \times 10^{4}$ \\
\hline${ }^{232} \mathrm{Th}$ & 25 & 17.771 & 12.0 & $1.41 \times 10^{10}$ \\
\hline${ }^{231} \mathrm{~Pa}$ & 29 & 2.885 & 12.0 & $3.28 \times 10^{4}$ \\
\hline${ }^{233} \mathrm{U}$ & 32 & 7.920 & $1.0^{c}$ & $1.59 \times 10^{5}$ \\
\hline${ }^{234} \mathrm{U}$ & 27 & 3.442 & 10.0 & $2.45 \times 10^{5}$ \\
\hline${ }^{235} \mathrm{U}$ & 28 & 8.531 & 1.0 & $7.04 \times 10^{8}$ \\
\hline${ }^{236} U$ & 26 & 7.906 & 5.0 & $2.34 \times 10^{7}$ \\
\hline${ }^{238} \mathrm{U}$ & 11 & 9.859 & 5.0 & $4.47 \times 10^{9}$ \\
\hline${ }^{238} \mathrm{Pu}$ & 30 & $2.687^{d}$ & $12.0^{c}$ & 87.7 \\
\hline${ }^{239} \mathrm{Pu}$ & 23 & 7.990 & 10.0 & $2.41 \times 10^{4}$ \\
\hline${ }^{240} \mathrm{Pu}$ & 22 & $10.537^{e}$ & 4.0 & $6.57 \times 10^{3}$ \\
\hline${ }^{240} \mathrm{Pu}$ & 21 & 10.782 & 3.0 & $6.57 \times 10^{3}$ \\
\hline${ }^{241} \mathrm{Pu}$ & 24 & $4.096^{e}$ & 2.0 & 14.36 \\
\hline${ }^{244} \mathrm{Pu}$ & 20 & 2.086 & $12.0^{c}$ & $8.05 \times 10^{7}$ \\
\hline${ }^{241} \mathrm{Am}$ & 15 & 9.551 & 1.0 & 432. \\
\hline${ }^{241} \mathrm{Am}$ & 14 & 10.383 & 0.1 & 432. \\
\hline${ }^{243} \mathrm{Am}$ & 12 & 9.804 & 0.2 & $7.37 \times 10^{3}$ \\
\hline${ }^{243} \mathrm{Cm}$ & 10 & $0.334^{\prime}$ & 1.0 & 28.5 \\
\hline${ }^{244} \mathrm{Cm}$ & 9 & $7.928^{g}$ & 2.0 & 18.1 \\
\hline${ }^{244} \mathrm{Cm}$ & 8 & $7.791^{\mathrm{g}}$ & $1.0^{c}$ & 18.1 \\
\hline${ }^{246} \mathrm{Cm}$ & 5 & 6.657 & 2.0 & $4.76 \times 10^{3}$ \\
\hline${ }^{248} \mathrm{Cm}$ & 4 & 1.720 & 10.0 & $3.50 \times 10^{5}$ \\
\hline
\end{tabular}

${ }^{a}$ From Table 13 of Ref. 1.

${ }^{b}$ From Table of Isotopes, Ref. 4.

'Specimen may have been damaged during its removal from vanadium capsule.

${ }^{d}$ Recomputed to be as of August 24, 1982, from data given in Table 13 of Ref. 1 and additional information given on page 50 of Ref. 3.

${ }^{e}$ Computed from compound weight given in Table 13 of Ref. 1 and additional information given on pages $40,41,42$, and 44 of Ref. 3.

${ }^{f}$ Recomputed to be as of August 24, 1982, from data given in Table 13 of Ref. 1 and additional information given on page 36 of Ref. 3.

${ }^{8}$ Recomputed to be as of August 24, 1982 from data given in Table 13 of Ref. 1 and additional information given on page 35 of - . 
Perusal of Table 2 will indicate that four samples prepared for the fuel pin (as described by Quinby et al. ${ }^{1}$ ) are missing from the list. These four samples are $\# 6$, the second ${ }^{246} \mathrm{Cm}$ sample; \#7, the ${ }^{237} \mathrm{~Np}$ sample; \#13, the second ${ }^{243} \mathrm{Am}$ sample; and \#16, the ${ }^{242} \mathrm{Pu}$ sample. The ${ }^{237} \mathrm{~Np}$ sample was reported as lost during the opening of the fuel pin following the irradiation. We did not receive a sample labelled \#13 (the second ${ }^{243} \mathrm{Am}$ sample) nor any explanation for its absence. As for the other two, we received labelled samples presumably containing our designated aliquots of the designated actinides, and gamma-ray data were obtained. However, the results of the gamma-ray measurements indicated that our sample \#6 contained a very substantial amount of ${ }^{243} \mathrm{Am}$ and was very likely the missing sample \#13. Because of the uncertainty of the assignment, coupled with apparently satisfactory data for sample \#12 (the first ${ }^{243} \mathrm{Am}$ sample), we chose to discard the data obtained from the sample labelled \#6. We also obtained data for a sample labelled \#16, but it became evident that the results were inconsistent with identification of the principal nuclide as ${ }^{242} \mathrm{Pu}$. In particular, we should have been rather readily able to identify gamma rays due to decay of ${ }^{242} \mathrm{Pu}$ and ${ }^{243} \mathrm{Am}$ and we were unable to do so. As a consequence it was apparent that the principal actinide was unlikely to be ${ }^{242} \mathrm{Pu}$, and as we were unable to identify the principal actinide, we (rather reluctantly) chose to discard the data obtained with this sample.

We had, also, another mixup in sample description. The sample labelled \#32, ${ }^{233} \mathrm{U}$, was determined to be ${ }^{230} \mathrm{Th}$, and the sample labelled $\# 31,{ }^{230} \mathrm{Th}$, was determined not to be ${ }^{230} \mathrm{Th}$. We decided the latter sample was the ${ }^{233} \mathrm{U}$ sample and treated the measurements and subsequent analyses for both samples on the assumption that the mixup was simply a labelling switch.

To summarize, fission-product yield data were obtained for fast-neutron fission of 19 different actinides. Samples of two actinides $\left({ }^{237} \mathrm{~Np}\right.$ and $\left.{ }^{242} \mathrm{Pu}\right)$ were apparently lost.

\subsection{GAMMA-RAY-ASSAY COUNTING CONFIGURATION}

Two different high-resolution detectors were used. One was a large-volume $\mathrm{Ge}(\mathrm{Li})$ diode having a resolution of $\sim 2.1 \mathrm{keV}$ full-width-at-half-maximum (FWHM) for $E_{\gamma} \sim 1.3 \mathrm{MeV}$. Measurements were made with this detector for $0.1 \leqslant E_{\gamma} \leqslant 3 \mathrm{MeV}$. The second detector was a low-energy-photon-system (LEPS) intrinsic germanium detector having a resolution of $\sim 0.5 \mathrm{keV}$ FWHM at $E_{\gamma} \sim 120 \mathrm{keV}$. It was used for measurements of gamma rays and $x$ rays having energies $20 \leqslant E_{\gamma} \leqslant 230 \mathrm{keV}$. The efficiencies of both detectors as functions of $E_{\gamma}$ were determined using well-calibrated commercially available sources.

The sample-to-detector configuration is shown schematically in Fig. 1. The glass bottle containing the (liquid) sample was oriented with its cylindrical axis set horizontally, and the bottle was fixed to a card (not shown) cut to fit into a "ladder" (also not shown) that has horizontal grooves cut at fixed distances, $D$, from the detector. Because of the extended nature of the source, several corrections had to be made, corrections not needed for the pointlike sources used during the efficiency calibration. The first correction was to the distance, $D$, between the bottom of the glass bottle and the detector. The glass bottles were $1.6 \mathrm{~cm}$ in outside diameter, had $\sim 1-\mathrm{mm}$ thick walls, and were $\sim 6-\mathrm{cm}$ long. The liquid filled approximately the lower half of the bottle, and we deduced the average path length of source gamma rays from somewhere in the liquid to the horizontal plane delineating the lowest side 


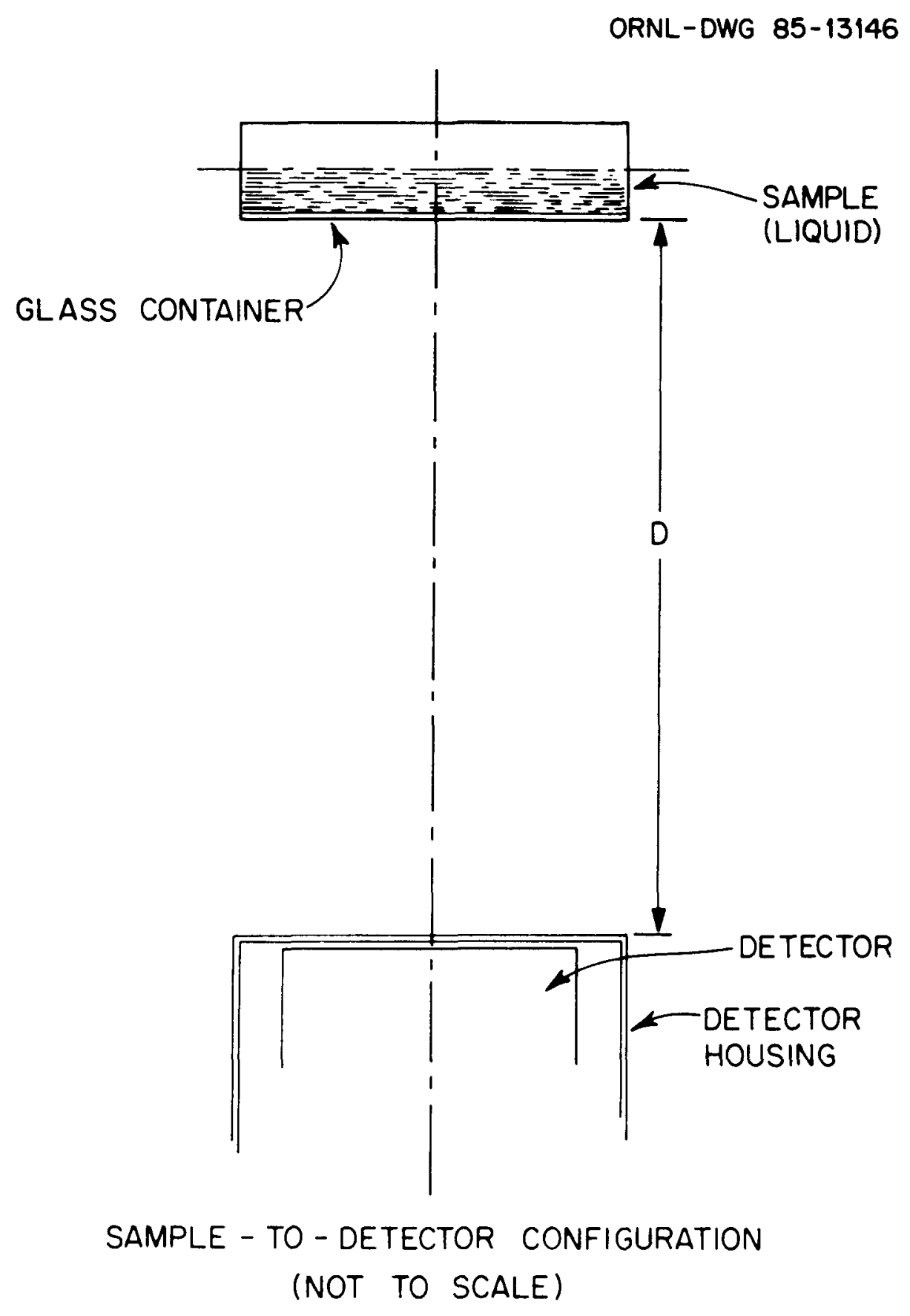

Fig. 1. Schematic representation of the sample orientation with respect to the $\mathbf{G e}(\mathbf{L i})$ (or intrinsic Ge) detector. The detector is mounted on a vertical dipstick and surrounded by lead shielding. A sample-holder ladder (not shown) made out of clear plastic is placed along the vertical centerline. This ladder has horizontal slots machined at known positions so as to provide an accurate value of the distance, $D$, from the top of the detector housing to the center of the slot. The sample is in liquid form and enclosed in a glass bottle permanently capped. The bottle is mounted on a piece of fiberboard card, and the card is positioned by use of the horizontal slots machined into the plastic ladder. 
of the (horizontally placed) bottle to be $0.5 \mathrm{~cm}$. The effect of the finite bottle length was computed. If $2 L=$ length of the bottle, then the average distance, $\rho(D, L)$, from a source on a horizontal line at a distance $D$ is given to first order by

$$
\rho(D, L)=\frac{1}{2} \sqrt{L^{2}+D^{2}}+\frac{D^{2}}{2 L} \quad\left[\ln \left(L+\sqrt{\left.L^{2}+D^{2}\right)}-\ln (D)\right] .\right.
$$

For $D=15 \mathrm{~cm}$ and $L=3 \mathrm{~cm}, \rho \sim 15.1 \mathrm{~cm}$, and the correction is small. For very nearly all measurements $D$ was $15 \mathrm{~cm}$ or larger for both detectors. One other correction that was applied was to correct for gamma-ray absorption by (a) the liquid of the sample, and (b) the $\mathrm{SiO}_{2}$ of the glass bottle. Gamma-ray attenuation coefficients were taken from the literature, ${ }^{5}$ and the computed attenuation corrections were checked by testing them on the measurements of one of the ${ }^{241} \mathrm{Am}$ samples, since the intensities of gamma rays due to decay of this radionuclide are well known. ${ }^{4}$

\subsection{GAMMA-RAY-ASSAY COUNTING MEASUREMENTS}

For each sample at least four separate spectra were obtained, two spectra during the period June to July 1984 and two spectra during November 1984. The two spectra for each period consisted of one spectrum for each of the two different detectors. For a few of the samples additional spectra were obtained during the period April to July 1985. These later spectra were obtained to attempt to provide answers to questions that arose during data reduction of the earlier spectra. All told, more than a hundred high-resolution gamma-ray spectra were obtained.

An example of spectra obtained with the LEPS detector is exhibited in Figs. 2 to 5 . In these figures, peaks are identified by symbols of the assigned responsible radionuclide. In addition, four positions are noted where peaks corresponding to the sample actinide, ${ }^{234} U$, would have been observed if the yields had been large enough. The fact that none of the four had a sufficient yield is consistent with amount of our sample as given in Table 2 and the relatively long half life of ${ }^{234} \mathrm{U}$. The only peak in the spectrum that gives an indication of the principal sample actinide is the comparatively small peak at $E_{\gamma} \sim 16 \mathrm{keV}$ (see Fig. 2), identified as probably due to thorium $L_{\beta}$ x-ray observation, a peak that would be expected following $\alpha$ decay of any one of the uranium isotopes. The fact that this $\mathrm{x}$ ray is observed implies an intermediate half life of the parent uranium isotope, and so is consistent with decay of the isotope ${ }^{234} \mathrm{U}$. This thorium peak is not observed, for example, in the spectrum of the sample of ${ }^{236} \mathrm{U}$.

An example of spectra obtained with the large-volume $\mathrm{Ge}(\mathrm{Li})$ detector is shown in Figs. 6 to 9 . Here again, peaks are identified by symbols of the responsible radionuclides. Essentially all of the observed peaks have been identified, although not all have been labelled. In Fig. 9, for example, all of the unlabelled peaks are due to detection of gamma rays following decay of ${ }^{106} \mathrm{Rh}$; however, only the peak at $E_{\gamma} \sim 2365 \mathrm{keV}$ (in this figure) was used as part of the determination of the yield of ${ }^{106} \mathrm{Rh}$. 


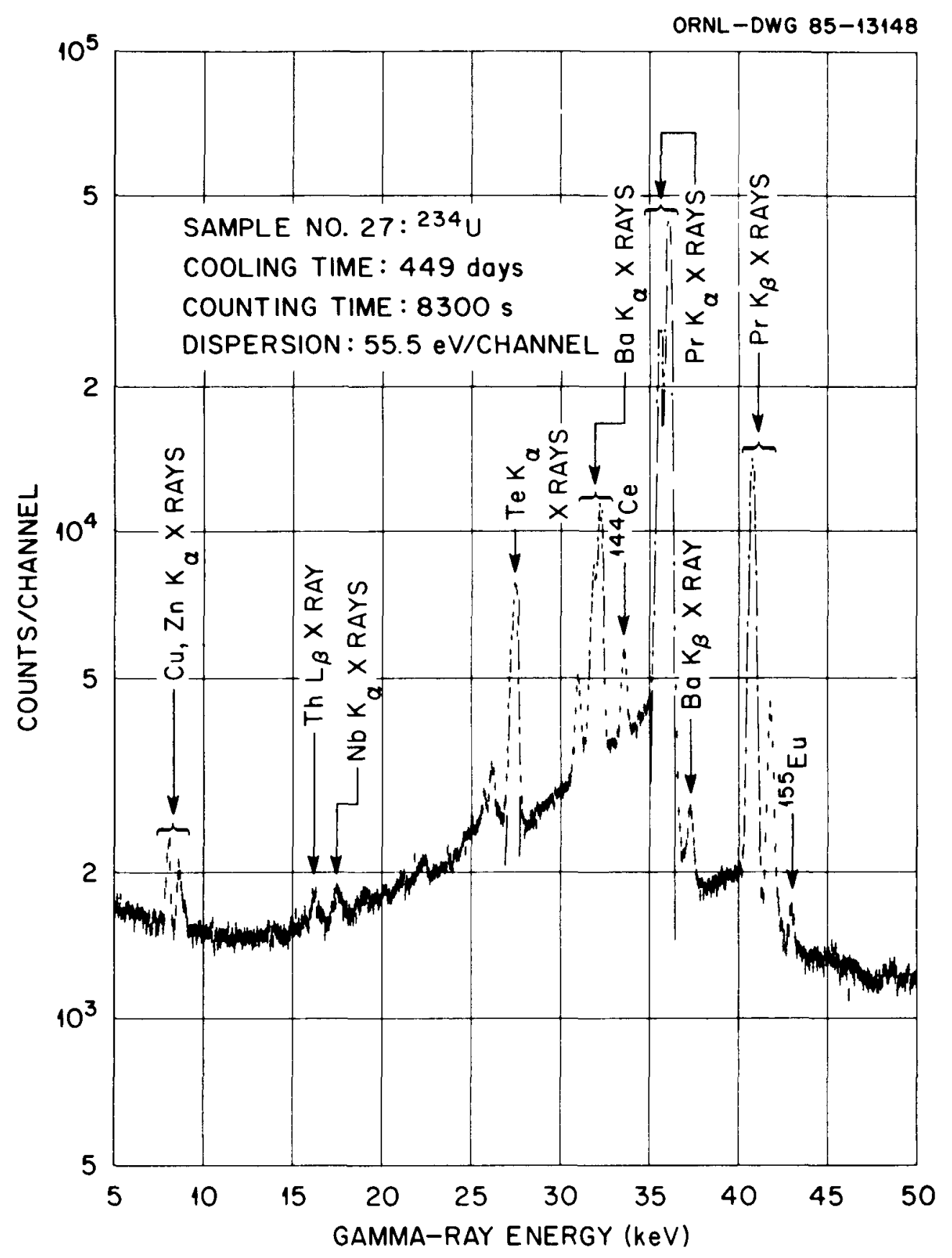

Fig. 2. Portion of the gamma-ray and x-ray spectrum obtained from a measurement of the ${ }^{234} U$ sample using the intrinsic-Ge low-energy photon detector. The copper and zinc $K_{\alpha} \times$ rays are due to flourescence by gamma rays of these elements in thin brass shielding pieces mounted on the front face of the more massive lead shielding. The small peaks at -26 and $31 \mathrm{keV}$ are germanium escape peaks. 


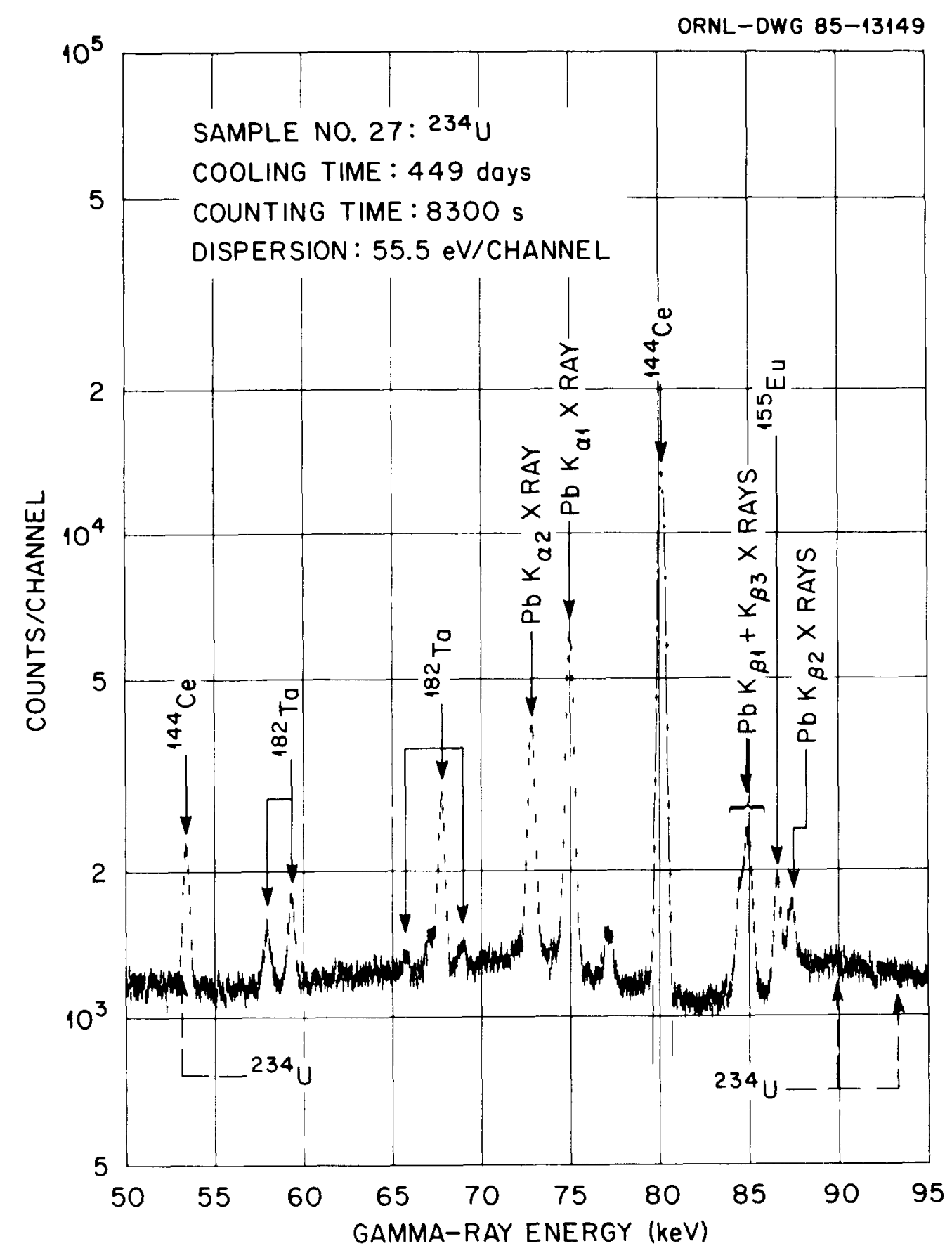

Fig. 3. Somewhat higher-energy portion of the spectrum shown in Fig. 2. The dashed lines indicate expected peak positions for detection of gamma rays following decay of ${ }^{234} \mathrm{U}$, the principal actinide of this sample. None are observed such that the mass of ${ }^{234} \mathrm{U}$ could be deduced. Also clearly observed are lead $K_{\alpha}$ and $K_{\beta}$ $x$ rays due to fluorescence by gamma rays of the lead in the shielding surrounding the detector. For the last measurements made, the shielding was revised to reduce this background source by a factor of more than 20. Such reduction was necessary to observe the $75-\mathrm{keV}$ "signature" gamma ray following decay of ${ }^{243} \mathrm{Am}$, particularly for measurements involving the heavier curium samples. 


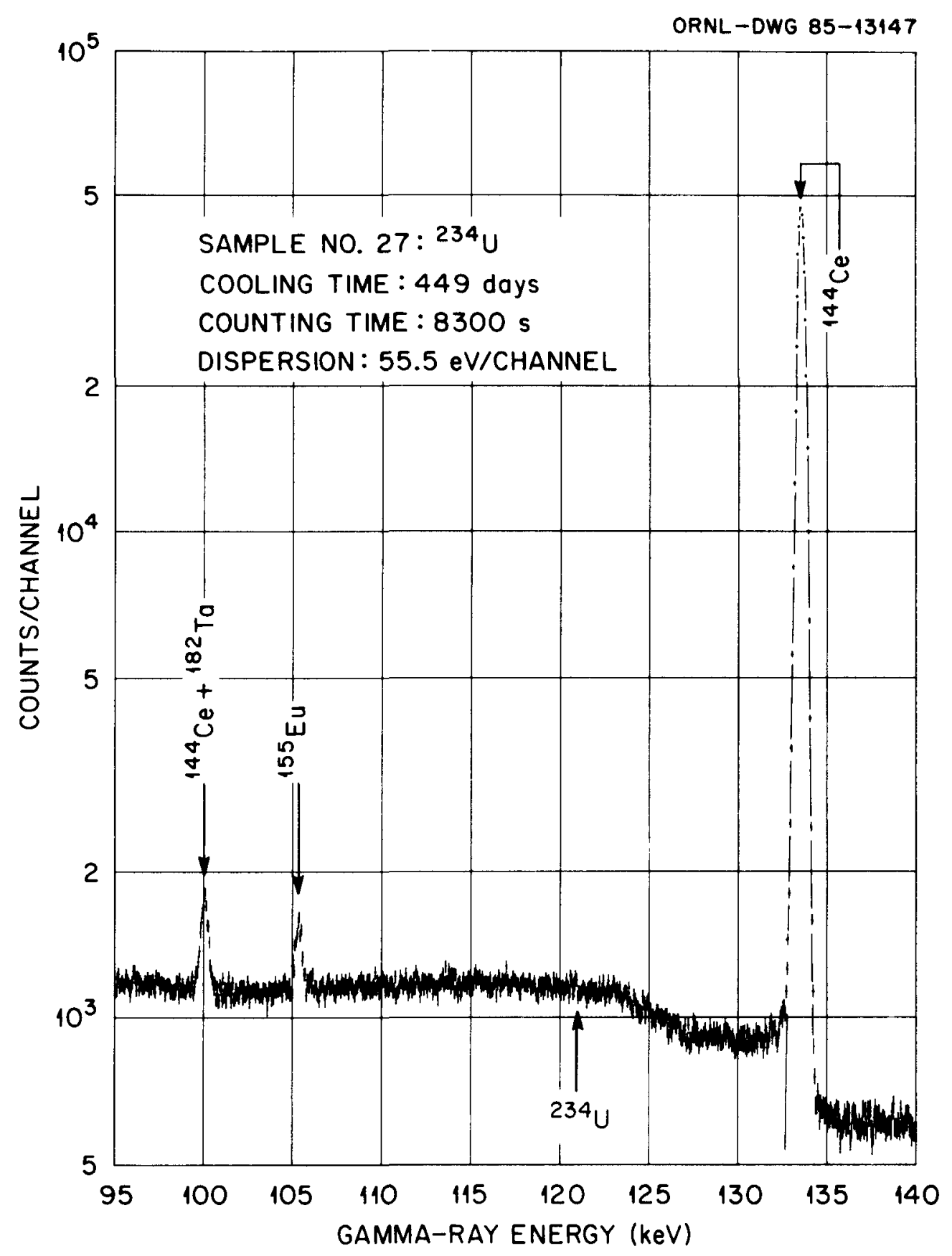

Fig. 4. Higher-energy portion of the spectrum of Figs. 2 and 3. 


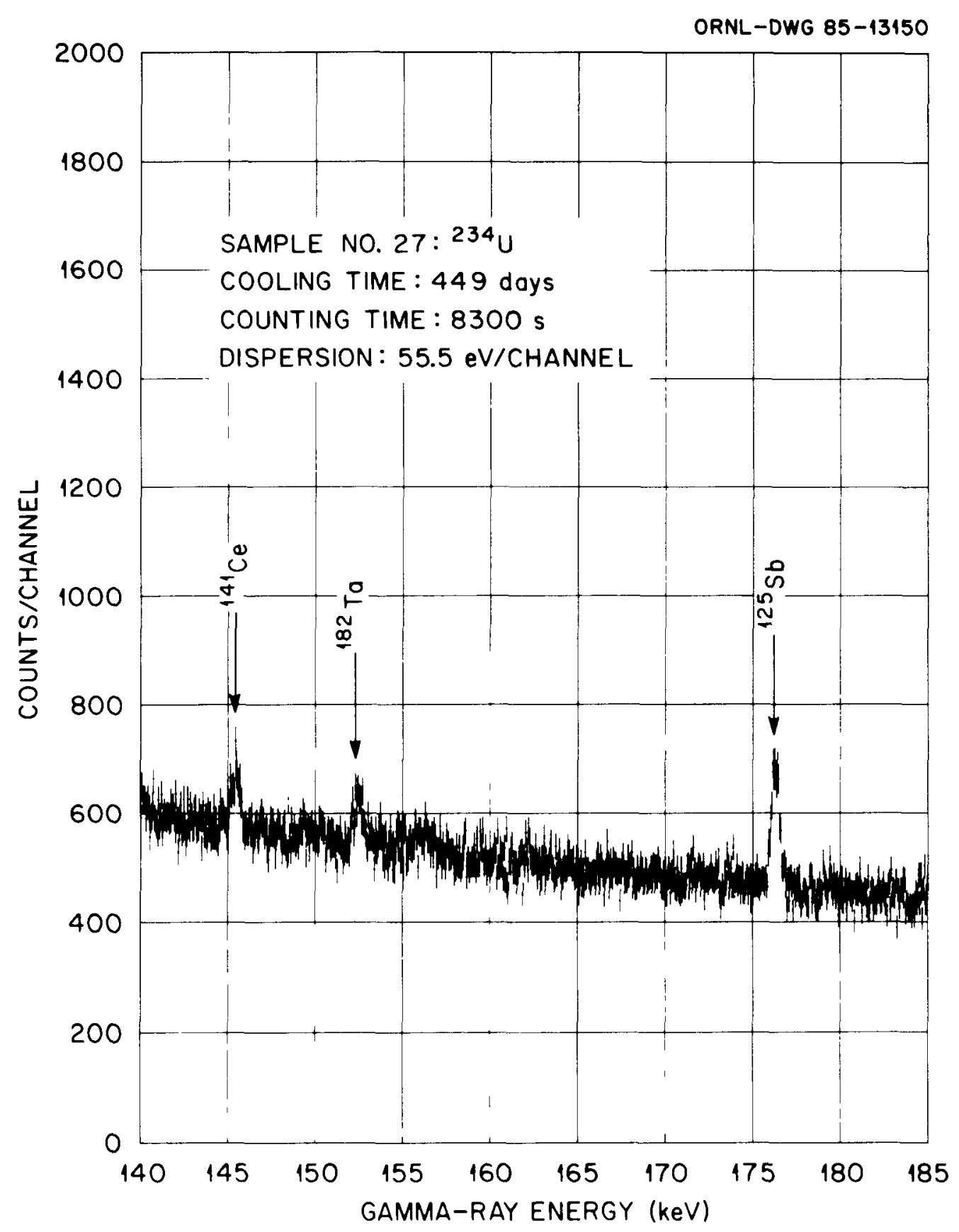

Fig. 5. Highest-energy portion of the spectrum of Figs. 2 to 4 . Data were obtained at larger energies, up to $230 \mathrm{keV}$, but no peaks corresponding to detection of gamma rays having energies $180 \mathrm{keV} \leqslant E_{\gamma} \leqslant 230 \mathrm{keV}$ were observed during the measurements involving the ${ }^{234} \mathrm{U}$ sample. 


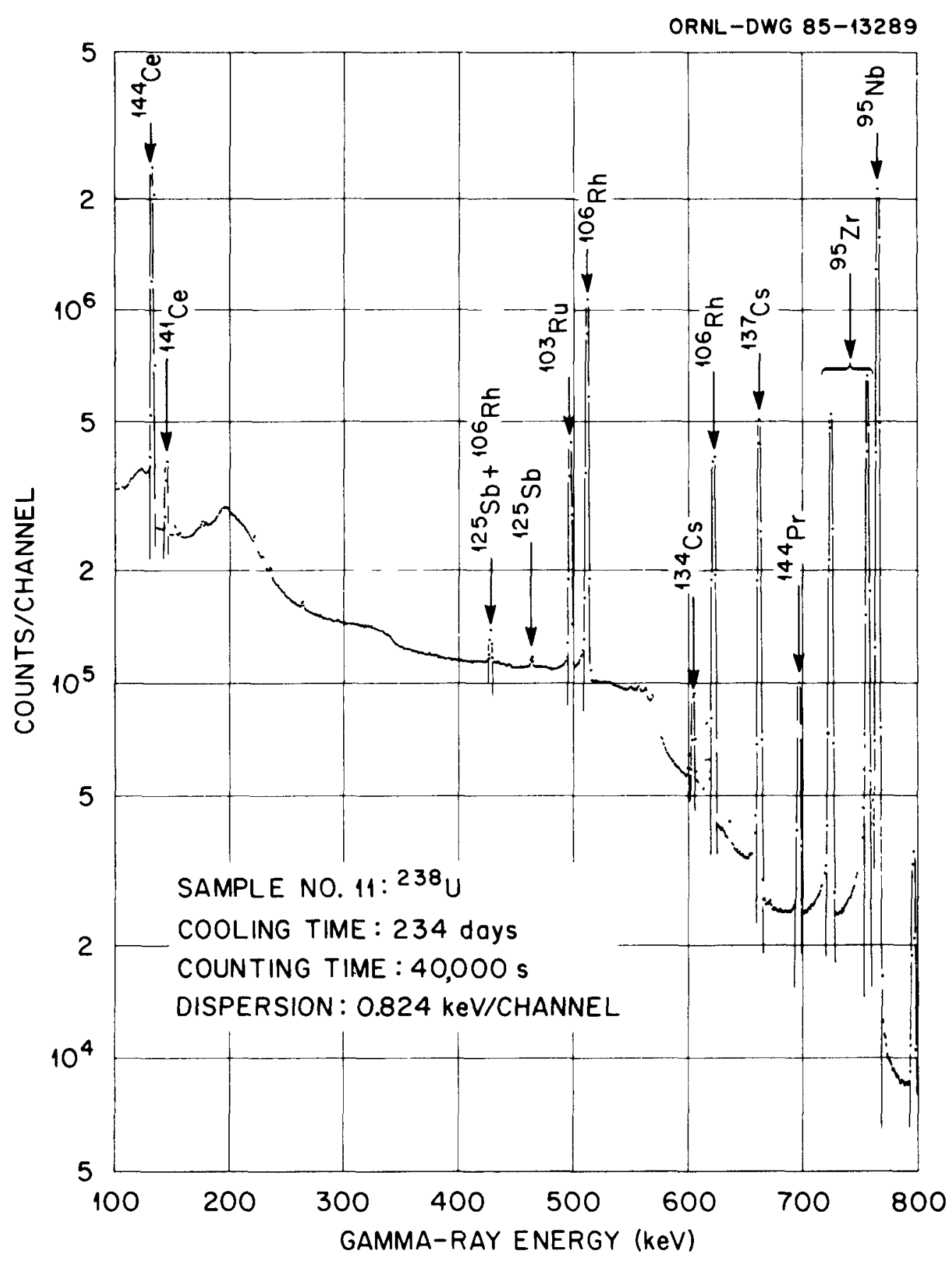

Fig. 6. Portion of the gamma-ray spectrum from a measurement of the ${ }^{238} \mathrm{U}$ sample using the large-volume $\mathrm{Ge}(\mathrm{Li})$ photon detector. All of the large peaks have been identified with gamma rays following decay of various fission products, and the peaks are labelled with isotopic symbols of the responsible radioisotopes. Resolution of this system may be judged by noting the clear separation of the $756-\mathrm{keV}$ gamma ray from decay of ${ }^{95} \mathrm{Zr}$ from the $765-\mathrm{keV}$ gamma ray from decay of ${ }^{95} \mathrm{Nb}$. The detector peak response is nearly Gaussian for almost all of the detectable portion of a given peak. The data-analysis code defines the peak shape as Gaussian plus a small low-energy contribution. 


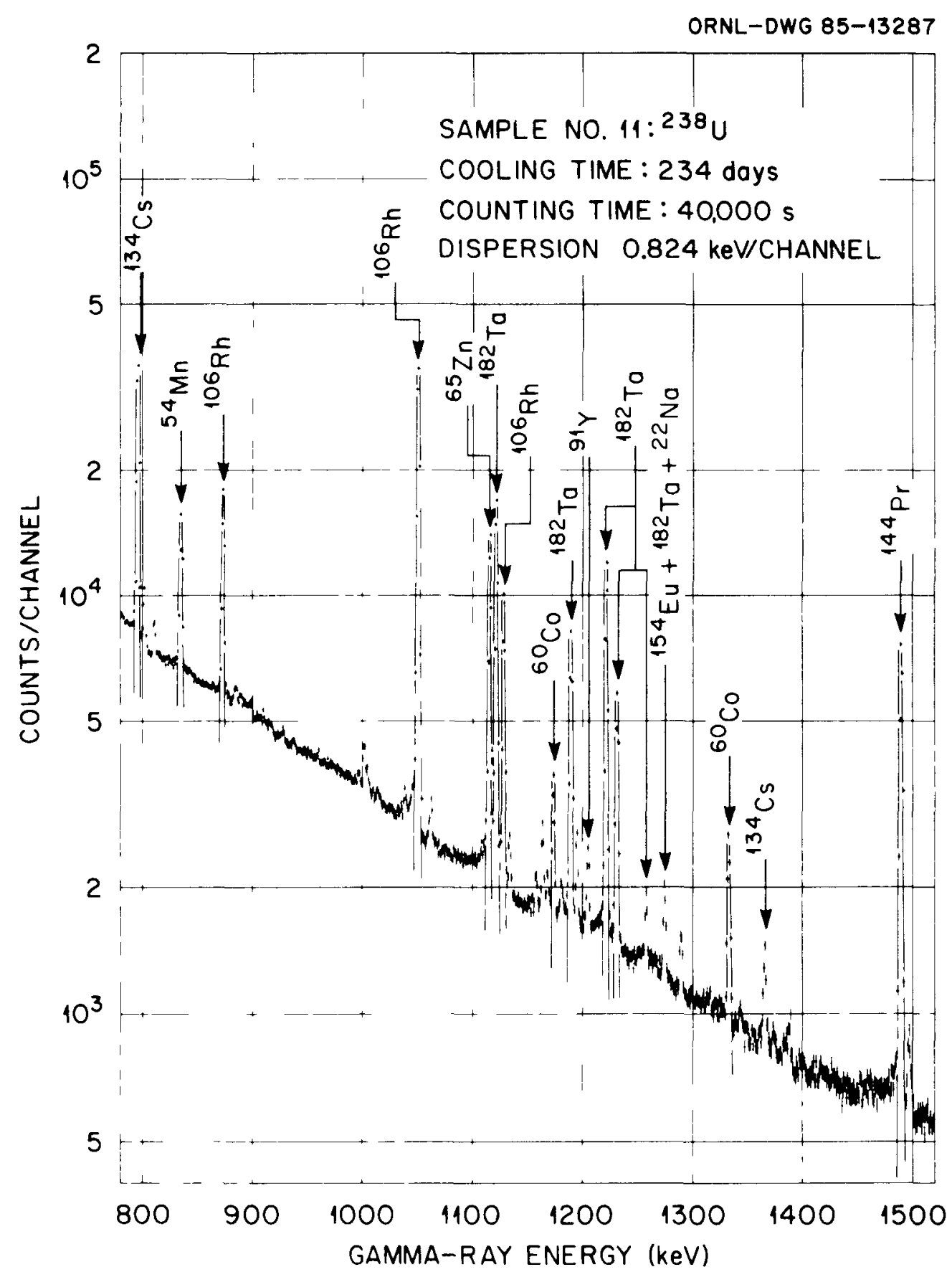

Fig. 7. Higher-energy portion of the spectrum of Fig. 6. As in that figure, isotopic symbols are used to indicate identifications of observed and analyzed peaks. Many of the small and unidentified peaks are due to detection of gamma rays following decay of ${ }^{106} \mathrm{Rh}$. 


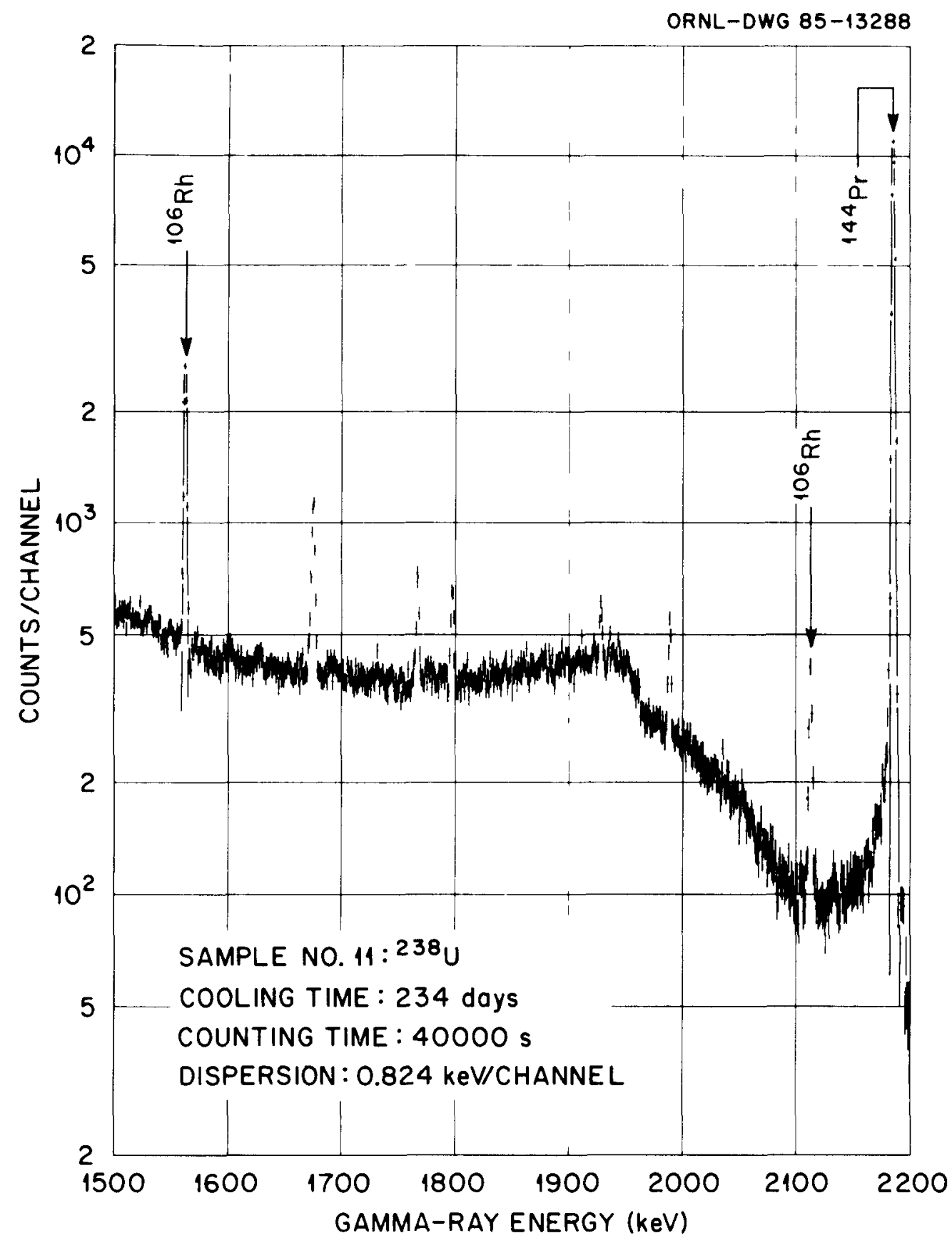

Fig. 8. Higher-energy portion of the spectrum of Figs. 6 and 7. The peak at $1674 \mathrm{keV}$ is due to the single escape of the $2185-\mathrm{keV}$ gamma ray from ${ }^{144} \mathrm{Pr}$ decay. The other four unidentified peaks are due to gamma rays following decay of ${ }^{106} \mathrm{Rh}$. 


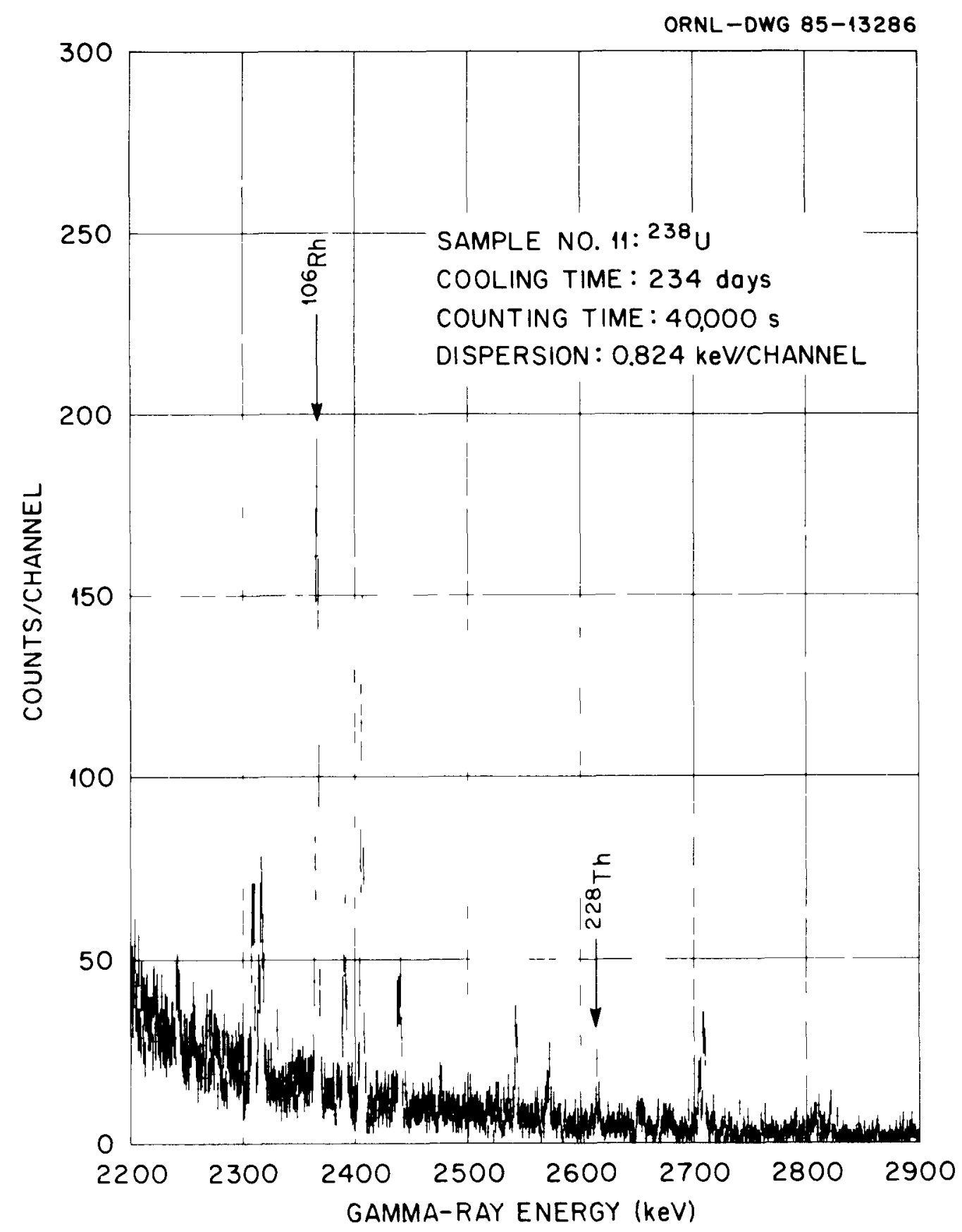

Fig. 9. Highest-energy portion of the spectrum of Figs. 6 to 8 . The peak at $2615 \mathrm{keV}$ is due to a gamma ray of that energy following decay of ${ }^{208} \mathrm{~T} \ell$, a daughter of ${ }^{228} \mathrm{Th}$ and a part of the room background. All of the remaining observed peaks are very likely due to gamma rays following decay of ${ }^{106} \mathrm{Rh}$. The one identified with the isotopic symbol was used during data reduction as part of the ${ }^{106} \mathrm{Rh}$ decay in deducing the yield of ${ }^{106} \mathrm{Rh}$; the other data were not so utilized. 
First-pass data reduction was accomplished using an up-to-date version of the well-tested computer code TPASS. ${ }^{6}$ Computed peak yields were corrected for detector efficiency and were then subjected to comparison with information stored in an isotope-data file ${ }^{7}$ to deduce likely responsible radionuclide candidates. In most instances, the analysis was unambiguous, but in those situations when the code located more than one likely contributing radionuclide, the final choice, or choices, had to be made by the experimentalist. In addition, the analysis for each run, as recorded in the print-out material, was carefully scanned for information that might have been passed over by the computer program.

The results of these calculations, both by computer and manually (when required), were in the units of the number of atoms of a specified fission product as of the end of the irradiation (EOI), taken as September 1, 1984. Of course, immediately at the end of the irradiation the actual yield of the specified fission product was slightly smaller, depending upon the existing amounts of other fission products which decayed into the specified fission product sometime after the end of the irradiation but prior to our measurements. The fission-product decay that we were able to observe had to be due to a few, comparatively long-lived fission products; the values reported here are for cumulative fission-product yields due to fast-neutron fission induced in each given sample for the actual irradiation history of the experiment.

In the described manner we were able to obtain yields for the following fission products: ${ }^{91} \mathrm{Y},{ }^{95} \mathrm{Zr},{ }^{95} \mathrm{Nb},{ }^{103} \mathrm{Ru},{ }^{106} \mathrm{Rh},{ }^{110 \mathrm{~m}} \mathrm{Ag},{ }^{125} \mathrm{Sb},{ }^{134} \mathrm{Cs},{ }^{137} \mathrm{Cs},{ }^{141} \mathrm{Ce},{ }^{144} \mathrm{Ce},{ }^{144} \mathrm{Pr}$, and ${ }^{155} \mathrm{Eu}$. We were not able to obtain information on certain other long-lived fission products anticipated in the preanalysis calculations of Broadhead $e t$ al. ${ }^{2}$ The radionuclide ${ }^{85} \mathrm{Kr}$ is a noble gas and was dispersed prior to our sample fabrication. The radionuclides ${ }^{89} \mathrm{Sr},{ }^{90} \mathrm{Sr}$, and ${ }^{90} \mathrm{Y}$ are, essentially, pure $\beta$-ray emitters, as are ${ }^{147} \mathrm{Pm}$ and ${ }^{151} \mathrm{Sm}$. Gamma rays may have been observed in some of the spectra corresponding to decay of other fission products, e.g., ${ }^{115} \mathrm{Cd},{ }^{129} \mathrm{Te},{ }^{148} \mathrm{Pm}$, and ${ }^{154} \mathrm{Eu}$.

Gamma radiations due to decay of several of the shorter-lived actinides were also observed. In some cases these "other" actinides were initially part of the sample, and in other cases they were created during the irradiation. Although not a part of the primary goal of the present experiment, these data were also reduced. In addition, gamma rays were observed due to decay of other radionuclides somehow introduced into the experimental samples but that were not created by neutron interactions with the principal (or any secondary) sample actinide. These "background" or "contaminant" radionuclides included ${ }^{54} \mathrm{Mn},{ }^{58} \mathrm{Co},{ }^{60} \mathrm{Co},{ }^{65} \mathrm{Zn}$, and, in particular, ${ }^{182} \mathrm{Ta}$. Identification of these background radionuclides was necessary so as to properly account for their contributions to peaks which corresponded to detection of gamma rays due to decay of radionuclides of interest.

\subsection{GAMMA-RAY-ASSAY COUNTING ANALYSES}

To obtain the absolute yields of radionuclides from the data given in the spectra requires knowledge of the radionuclide half lives and individual gamma-ray decay emission probabilities (known as branching ratios). Values for the nuclear data ${ }^{4,6}$ that we used are given in Table 3 for the fission products of interest. The radionuclides given in this table have been well studied, and one may consider most of the given nuclear data as reliable. Uncertainties associated with half lives were not propagated in the computations; however, uncertainties in branching ratios were included in the final uncertainty determinations. 
Table 3. Nuclear data for fission products

\begin{tabular}{|c|c|c|c|}
\hline \multirow[b]{2}{*}{ Radionuclide } & \multirow[b]{2}{*}{ Half-life } & \multicolumn{2}{|c|}{ Gamma-ray } \\
\hline & & Energy (keV) & Branching (\%) \\
\hline${ }^{91} \mathrm{Y}$ & $58.5 \mathrm{~d}$ & 1204.9 & $0.30 \pm 0.03$ \\
\hline${ }^{95} \mathrm{Zr}$ & $64.0 \mathrm{~d}$ & $\begin{array}{l}724.24 \\
756.76\end{array}$ & $\begin{array}{l}44.2 \pm 0.5 \\
54.6 \pm 0.5\end{array}$ \\
\hline${ }^{95} \mathrm{Nb}$ & $35.0 \mathrm{~d}$ & 765.84 & $99.8 \pm 0.1$ \\
\hline${ }^{103} \mathrm{Ru}$ & $39.3 \mathrm{~d}$ & 497.04 & $90.9 \pm 0.7$ \\
\hline${ }^{106} \mathrm{Rh}$ & $366.9 \mathrm{~d}^{a}$ & $\begin{array}{r}621.8 \\
1050.1\end{array}$ & $\begin{array}{r}9.95 \pm 0.75 \\
1.45 \pm 0.10\end{array}$ \\
\hline${ }^{110 \mathrm{~m}} \mathrm{Ag}^{b}$ & $252 \mathrm{~d}$ & $\begin{array}{r}884.2 \\
937.4 \\
1383.9\end{array}$ & $\begin{array}{l}72.6 \pm 0.3 \\
34.2 \pm 0.3 \\
24.3 \pm 0.2\end{array}$ \\
\hline${ }^{125} \mathrm{Sb}$ & $2.758 \mathrm{y}$ & $\begin{array}{l}427.89^{c} \\
600.56 \\
635.90 \\
463.38\end{array}$ & $\begin{array}{l}29.44 \pm 0.99 \\
17.78 \pm 0.95 \\
11.32 \pm 0.68 \\
10.45 \pm 0.57\end{array}$ \\
\hline${ }^{134} \mathrm{Cs}$ & $2.066 \mathrm{y}$ & $\begin{array}{l}604.74 \\
795.80\end{array}$ & $\begin{array}{l}98.6 \pm 0.3 \\
87.8 \pm 1.4\end{array}$ \\
\hline${ }^{137} \mathrm{Cs}$ & $30.14 \mathrm{y}$ & $661.64^{d}$ & $86.0 \pm 0.6^{d}$ \\
\hline${ }^{141} \mathrm{Ce}$ & $32.5 \mathrm{~d}$ & 145.45 & $48.3 \pm 0.3$ \\
\hline${ }^{144} \mathrm{Ce}$ & $284.7 \mathrm{~d}$ & 133.53 & $11.09 \pm 0.20$ \\
\hline${ }^{144} \mathrm{Pr}$ & $284.7 \mathrm{~d}^{e}$ & $\begin{array}{r}696.48 \\
2185.78\end{array}$ & $\begin{array}{l}1.34 \pm 0.02 \\
0.74 \pm 0.03\end{array}$ \\
\hline${ }^{155} \mathrm{Eu}$ & $4.96 \mathrm{y}$ & $\begin{array}{r}86.55 \\
105.31\end{array}$ & $\begin{array}{l}30.9 \pm 2.8 \\
20.7 \pm 2.0\end{array}$ \\
\hline
\end{tabular}

${ }^{a}$ Of the parent ${ }^{106} \mathrm{Ru}$.

${ }^{b}$ The peak corresponding to detection of the $E_{\gamma}=$ $657.7-\mathrm{keV}$ gamma ray from decay of this isotope is masked by the very much larger peak similarly corresponding to decay of ${ }^{137} \mathrm{Cs}$. ${ }^{106} \mathrm{Rh}$

'Nearly degenerate with $E_{\gamma}=428.4 \mathrm{keV}$ due to decay of

${ }^{d}$ Gamma ray due to decay of daughter ${ }^{137} \mathrm{Ba}$ isomer; branching corrected to be applicable to decay of ${ }^{137} \mathrm{Cs}$.

eOf the parent ${ }^{144} \mathrm{Ce}$. 
The results were obtained as the absolute values of the number of atoms of the particular radionuclide that were in the sample at the time of the gamma-ray measurement. In Table 4 results for ${ }^{137} \mathrm{Cs}$ are tabulated. Note that, as obtained and presented, the given results for ${ }^{137} \mathrm{Cs}$ are not related to any information about the sample material, irradiation history, etc. The uncertainties tabulated with the absolute yields include data reduction uncertainties, which are dominated by uncertainties in peak area determinations. The tabulated uncertainties do not include uncertainties in the detector efficiency at $E_{\gamma}=662 \mathrm{keV}$, which, in turn depend upon the accuracy with which the sample-to-detector distance was determined for a given measurement. For point-source measurements this distance is believed known to $\pm 0.1 \mathrm{~cm}$, which would correspond to an uncertainty of $\sim 1.5 \%$ for $D=15 \mathrm{~cm}$, which should be quadratically combined with an uncertainty of $2 \%$ assigned to the experimental efficiency at $E_{\gamma}=662 \mathrm{keV}$ for $D=15 \mathrm{~cm}$. The tabulated uncertainties assigned to the data given in Table 4 range between $-1 \%$ and $\sim 3 \%$. Comparing the November 1984 measurements with the earlier measurements indicates that the former disagree with the latter by more than the combined uncertainties for 10 of the 22 samples, a somewhat larger number of disagreements than might be expected. We noted, however, that such disagreements as were observed were reflected in overall normalization of a given measurement, such that the ratios of the yields of the other observed fission products to that for ${ }^{137} \mathrm{Cs}$ did not vary near as much as did the absolute yields of ${ }^{137} \mathrm{Cs}$.

As given in Table 4, of course, the yields for ${ }^{137} \mathrm{Cs}$ must be further interpreted. The reduction of these results to quantities of physical interest is discussed in the next section.

Before going on to the next section, however, we give a brief discussion of the various background radionuclide decays that were observed. As mentioned above, data were observed which could be attributed to decay of several non-fission-product and non-actinide radionuclides, in particular ${ }^{182} \mathrm{Ta}$. These results are given in Table 5. One may observe that the reported values span four orders of magnitude. One may also observe an approximate correlation in yields of ${ }^{54} \mathrm{Mn},{ }^{58} \mathrm{Co}$, and ${ }^{60} \mathrm{Co}$, but much less correlation of the yields of these three radionuclides with either ${ }^{65} \mathrm{Zn}$ or ${ }^{182} \mathrm{Ta}$. As mentioned above, the impurity yields were important for the data reduction, particularly for ${ }^{182} \mathrm{Ta}$. Of some concern, pertaining to sample descriptions, was the source (or sources) of the impurities. Clearly they were in our aliquots. The substantial variations from one sample to another suggested that the impurities were not inadvertently added from extraneous sources during our sample preparations. Hence, it appeared to us that the impurities were in the samples. So the impurities in the samples as deduced using spark source mass spectrographic methods were scanned (see the report of Walker et $a l^{3}{ }^{3}$ ). Some of these SSMS impurity results are given in Table 6, and one may ask if the reported ${ }^{3}$ impurity amounts can account for our observations. The observed ${ }^{60} \mathrm{Co}$ may be expected to correlate with the SSMS-deduced Co impurity. The largest reported Coimpurity amount is for ${ }^{236} \mathrm{U}$, and for this sample [using $\sigma(n, \gamma)$ for ${ }^{59} \mathrm{Co}$ of $0.1232 \mathrm{~b}$ taken from Ref. 2, Table 20, page 27] one may estimate a production of $8.4 \times 10^{11}$ atoms of ${ }^{60} \mathrm{Co}$ for our aliquot of the sample, a value which is larger than the observed $1.9 \times 10^{11}$ atoms of ${ }^{60} \mathrm{Co}$. For ${ }^{231} \mathrm{~Pa}$ and for ${ }^{241} \mathrm{Am}$ (\#15) the estimates from SSMS-given cobalt-impurity values are $4.4 \times 10^{10}$ and $2.6 \times 10^{10}$ atoms, respectively, which are somewhat small compared to the observed values of $5.1 \times 10^{11}$ and $1.6 \times 10^{11}$ atoms, respectively. For the remaining principal actinides the computed estimate of expected ${ }^{60} \mathrm{Co}$ is more than an order of magnitude smaller than observed. The conclusion appears to be that the observed amounts of ${ }^{60} \mathrm{Co}$ are larger than expected from and not particularly well correlated with the given impurity 
Table 4. Absolute experimental yields of the fission product ${ }^{137} \mathrm{Cs}$ in numbers of atoms ${ }^{a}$ as of the end of the PFR irradiation (September 1, 1983)

\begin{tabular}{|c|c|c|c|c|}
\hline \multirow{2}{*}{$\begin{array}{c}\text { Principal } \\
\text { isotope }\end{array}$} & \multirow{2}{*}{$\begin{array}{c}\text { Sample } \\
\text { No. }\end{array}$} & \multicolumn{3}{|c|}{ Measurement periods } \\
\hline & & June-July, 1984 & November 1984 & April-July 1985 \\
\hline${ }^{230} \mathrm{Th}$ & 31 & $(8.39 \pm 0.08) \times 10^{12}$ & $(7.95 \pm 0.08) \times 10^{12}$ & $(8.39 \pm 0.08) \times 10^{12}$ \\
\hline${ }^{232} \mathrm{Th}$ & 25 & $(1.164 \pm 0.015) \times 10^{14}$ & $(1.178 \pm 0.012) \times 10^{14}$ & \\
\hline${ }^{231} \mathrm{~Pa}$ & 29 & $(2.129 \pm 0.023) \times 10^{14}$ & $(2.230 \pm 0.031) \times 10^{14}$ & $(2.162 \pm 0.034) \times 10^{14}$ \\
\hline${ }^{233} \mathrm{U}$ & 32 & $(6.32 \pm 0.10) \times 10^{14}$ & $(6.43 \pm 0.08) \times 10^{14}$ & \\
\hline${ }^{234} \mathrm{U}$ & 27 & $(3.38 \pm 0.07) \times 10^{14}$ & $(3.45 \pm 0.04) \times 10^{14}$ & $(3.45 \pm 0.05) \times 10^{14}$ \\
\hline${ }^{235} \mathrm{U}$ & 28 & $(4.28 \pm 0.12) \times 10^{14}$ & $(4.55 \pm 0.06) \times 10^{14}$ & \\
\hline${ }^{236} \mathrm{U}$ & 26 & $(3.45 \pm 0.07) \times 10^{14}$ & $(3.51 \pm 0.04) \times 10^{14}$ & \\
\hline${ }^{238} \mathrm{U}$ & 11 & $(8.30 \pm 0.12) \times 10^{13}$ & $(8.45 \pm 0.09) \times 10^{13}$ & \\
\hline${ }^{238} \mathrm{Pu}$ & 30 & $(1.434 \pm 0.016) \times 10^{14}$ & $(1.388 \pm 0.016) \times 10^{14}$ & \\
\hline${ }^{239} \mathrm{Pu}$ & 23 & $(1.899 \pm 0.029) \times 10^{14}$ & $\left(1.862 \pm 0.0300 \times 10^{14}\right.$ & \\
\hline${ }^{240} \mathrm{Pu}$ & 22 & $(5.15 \pm 0.07) \times 10^{14}$ & $(4.99 \pm 0.06) \times 10^{14}$ & \\
\hline${ }^{240} \mathrm{Pu}$ & 21 & $(3.72 \pm 0.07) \times 10^{14}$ & $(3.90 \pm 0.05) \times 10^{14}$ & \\
\hline${ }^{241} \mathrm{Pu}$ & 24 & $(4.38 \pm 0.06) \times 10^{14}$ & $(4.37 \pm 0.06) \times 10^{14}$ & \\
\hline${ }^{244} \mathrm{Pu}$ & 20 & $(1.991 \pm 0.028) \times 10^{14}$ & $(1.994 \pm 0.023) \times 10^{14}$ & \\
\hline${ }^{241} \mathrm{Am}$ & 15 & $(8.72 \pm 0.16) \times 10^{13}$ & $(8.73 \pm 0.09) \times 10^{13}$ & \\
\hline${ }^{241} \mathrm{Am}$ & 14 & $(1.008 \pm 0.010) \times 10^{13}$ & $(9.92 \pm 0.10) \times 10^{12}$ & \\
\hline${ }^{243} \mathrm{Am}$ & 12 & $(1.352 \pm 0.013) \times 10^{13}$ & $(1.244 \pm 0.013) \times 10^{13}$ & \\
\hline${ }^{243} \mathrm{Cm}$ & 10 & $(4.88 \pm 0.09) \times 10^{13}$ & $(4.35 \pm 0.13) \times 10^{13}$ & \\
\hline${ }^{244} \mathrm{Cm}$ & 9 & $(2.411 \pm 0.042) \times 10^{14}$ & $(2.552 \pm 0.035) \times 10^{14}$ & \\
\hline${ }^{244} \mathrm{Cm}$ & 8 & $(6.41 \pm 0.07) \times 10^{13}$ & & \\
\hline${ }^{246} \mathrm{Cm}$ & 5 & $(2.336 \pm 0.027) \times 10^{14}$ & $(2.053 \pm 0.022) \times 10^{14}$ & \\
\hline${ }^{248} \mathrm{Cm}$ & 4 & $(1.714 \pm 0.022) \times 10^{14}$ & $(1.714 \pm 0.020) \times 10^{14}$ & \\
\hline
\end{tabular}

${ }^{a}$ Uncertainties are data analysis uncertainties and do not include uncertainties in overall detection efficiency for $E_{\gamma}=662 \mathrm{keV}$, estimated at $\pm 3 \%$, nor that associated with the glass wall attenuation, estimated at $<1 \%$. 
Table 5. Background radionuclide decay observed. Units are number

of atoms as of the end of the irradiation (September 1, 1983).

\begin{tabular}{|c|c|c|c|c|c|c|}
\hline $\begin{array}{c}\text { Principal } \\
\text { isotope }\end{array}$ & $\begin{array}{c}\text { Sample } \\
\text { no. }\end{array}$ & ${ }^{54} \mathrm{Mn}$ & ${ }^{58} \mathrm{Co}$ & ${ }^{60} \mathrm{Co}$ & ${ }^{65} \mathrm{Zn}$ & ${ }^{182} \mathrm{Ta}$ \\
\hline${ }^{230} \mathrm{Th}$ & 31 & $(4.40 \pm 0.04) \times 10^{11}$ & $(3.25 \pm 0.06) \times 10^{11}$ & $(6.75 \pm 0.08) \times 10^{11}$ & $(7.6 \pm 2.1) \times 10^{9}$ & $(9.05 \pm 0.11) \times 10^{11}$ \\
\hline${ }^{232} \mathrm{Th}$ & 25 & $(1.87 \pm 0.04) \times 10^{11}$ & $(5.8 \pm 0.8) \times 10^{10}$ & $(2.40 \pm 0.15) \times 10^{11}$ & $(2.95 \pm 0.20) \times 10^{10}$ & $(3.46 \pm 0.12) \times 10^{11}$ \\
\hline${ }^{231} \mathrm{~Pa}$ & 29 & $(3.30 \pm 0.06) \times 10^{11}$ & $(7.0 \pm 1.2) \times 10^{11}$ & $(5.1 \pm 0.5) \times 10^{11}$ & $(3.60 \pm 0.08) \times 10^{11}$ & $(1.97 \pm 0.08) \times 10^{12}$ \\
\hline${ }^{233} \mathrm{U}$ & 32 & $(4.82 \pm 0.08) \times 10^{10}$ & & $(6.5 \pm 0.4) \times 10^{10}$ & $(5.5 \pm 2.2) \times 10^{10}$ & $(4.85 \pm 0.11) \times 10^{12}$ \\
\hline${ }^{234} \mathrm{U}$ & 27 & $(1.50 \pm 0.03) \times 10^{11}$ & $(6.8 \pm 0.4) \times 10^{10}$ & $(4.20 \pm 0.22) \times 10^{11}$ & $(1.87 \pm 0.06) \times 10^{11}$ & $(1.45 \pm 0.04) \times 10^{12}$ \\
\hline${ }^{235} \mathrm{U}$ & 28 & $(1.6 \pm 0.8) \times 10^{10}$ & & $(2.0 \pm 1.7) \times 10^{10}$ & & $(6.4 \pm 3.1) \times 10^{10}$ \\
\hline${ }^{236} U$ & 26 & $(6.28 \pm 0.28) \times 10^{11}$ & & $(1.94 \pm 0.17) \times 10^{11}$ & $(2.35 \pm 0.16) \times 10^{11}$ & $(7.07 \pm 0.14) \times 10^{11}$ \\
\hline${ }^{238} \mathrm{U}$ & 11 & $(8.70 \pm 0.13) \times 10^{10}$ & $(6.7 \pm 0.5) \times 10^{9}$ & $(1.01 \pm 0.07) \times 10^{11}$ & $(2.92 \pm 0.08) \times 10^{11}$ & $(6.05 \pm 0.20) \times 10^{11}$ \\
\hline${ }^{238} \mathrm{Pu}$ & 30 & $(5.44 \pm 0.11) \times 10^{11}$ & $(4.55 \pm 0.07) \times 10^{11}$ & $(7.60 \pm 0.10) \times 10^{11}$ & $(9.2 \pm 0.9) \times 10^{9}$ & $(3.25 \pm 0.25) \times 10^{11}$ \\
\hline${ }^{239} \mathrm{Pu}$ & 23 & $(8.42 \pm 0.13) \times 10^{13}$ & $(7.74 \pm 0.11) \times 10^{13}$ & $(8.51 \pm 0.12) \times 10^{13}$ & $(9 \pm 3) \times 10^{10}$ & $(2.05 \pm 0.30) \times 10^{11}$ \\
\hline${ }^{240} \mathrm{Pu}$ & 22 & $(2.06 \pm 0.04) \times 10^{11}$ & & $(1.90 \pm 0.22) \times 10^{11}$ & $(4.3 \pm 0.2) \times 10^{9}$ & $(3 \pm 2) \times 10^{10}$ \\
\hline${ }^{240} \mathrm{Pu}$ & 21 & $(2.21 \pm 0.06) \times 10^{11}$ & $(1.24 \pm 0.05) \times 10^{11}$ & $(1.85 \pm 0.11) \times 10^{11}$ & $(7 \pm 5) \times 10^{9}$ & $(7.7 \pm 0.8) \times 10^{10}$ \\
\hline${ }^{241} \mathrm{Pu}$ & 24 & $(1.82 \pm 0.03) \times 10^{11}$ & & $(5.1 \pm 0.9) \times 10^{10}$ & $(3.5 \pm 0.4) \times 10^{10}$ & $(3 \pm 1) \times 10^{10}$ \\
\hline${ }^{244} \mathrm{Pu}$ & 20 & $(3.48 \pm 0.05) \times 10^{12}$ & $(2.64 \pm 0.04) \times 10^{12}$ & $(2.99 \pm 0.05) \times 10^{12}$ & $(5.06 \pm 0.22) \times 10^{10}$ & $(1.15 \pm 0.05) \times 10^{12}$ \\
\hline${ }^{241} \mathrm{Am}$ & 15 & $(9.8 \pm 0.4) \times 10^{10}$ & $(6.9 \pm 0.6) \times 10^{10}$ & $(5.5 \pm 0.5) \times 10^{10}$ & $(1.65 \pm 0.07) \times 10^{11}$ & $(7.3 \pm 0.8) \times 10^{10}$ \\
\hline${ }^{241} \mathrm{Am}$ & 14 & $(5.83 \pm 0.17) \times 10^{9}$ & $(1.0 \pm 0.1) \times 10^{10}$ & $(6.2 \pm 1.6) \times 10^{9}$ & $(2.5 \pm 0.2) \times 10^{10}$ & $(2.3 \pm 0.2) \times 10^{9}$ \\
\hline${ }^{243} \mathrm{Am}$ & 12 & $(1.86 \pm 0.09) \times 10^{10}$ & & $(3.9 \pm 0.9) \times 10^{9}$ & $(4.6 \pm 0.6) \times 10^{9}$ & $(5.5 \pm 0.5) \times 10^{9}$ \\
\hline${ }^{243} \mathrm{Cm}$ & 10 & $(1.3 \pm 0.4) \times 10^{10}$ & & $(2.3 \pm 0.3) \times 10^{10}$ & $(6 \pm 4) \times 10^{9}$ & $(2.7 \pm 0.3) \times 10^{10}$ \\
\hline${ }^{244} \mathrm{Cm}$ & 9 & $(3.64 \pm 0.19) \times 10^{10}$ & & $(5.1 \pm 0.3) \times 10^{9}$ & $(1.37 \pm 0.13) \times 10^{11}$ & $(2.78 \pm 0.19) \times 10^{11}$ \\
\hline${ }^{244} \mathrm{Cm}$ & 8 & $(1.05 \pm 0.04) \times 10^{10}$ & $(8.1 \pm 1.3) \times 10^{9}$ & $(1.38 \pm 0.09) \times 10^{10}$ & $(1.26 \pm 0.05) \times 10^{11}$ & $(1.06 \pm 0.05) \times 10^{11}$ \\
\hline${ }^{246} \mathrm{Cm}$ & 5 & $(4.8 \pm 0.9) \times 10^{10}$ & & $(3.2 \pm 1.3) \times 10^{10}$ & $(8.8 \pm 0.4) \times 10^{10}$ & $(3.34 \pm 0.07) \times 10^{11}$ \\
\hline${ }^{248} \mathrm{Cm}$ & 4 & $(3.31 \pm 0.05) \times 10^{11}$ & $(1.30 \pm 0.05) \times 10^{11}$ & $(2.45 \pm 0.25) \times 10^{11}$ & $(2.62 \pm 0.12) \times 10^{11}$ & $(1.36 \pm 0.03) \times 10^{12}$ \\
\hline
\end{tabular}


Table 6. SSMS selected impurity analyses of actinide samples. ${ }^{a}$ Units are elemental mass ratio in parts per million.

\begin{tabular}{|c|c|c|c|c|c|}
\hline \multirow{2}{*}{$\begin{array}{c}\text { Principal } \\
\text { isotope }\end{array}$} & \multicolumn{5}{|c|}{ Impurity element } \\
\hline & $\mathrm{Fe}$ & $\mathrm{Na}$ & $\mathrm{Zr}$ & Co & $\mathrm{Zn}$ \\
\hline${ }^{230} \mathrm{Th}$ & 10 & 100 & & & \\
\hline${ }^{232} \mathrm{Th}$ & 100 & 0.3 & 0.3 & 0.3 & 3 \\
\hline${ }^{231} \mathrm{~Pa}$ & 1000 & 1000 & 10 & 5 & 100 \\
\hline${ }^{233} \mathrm{U}$ & 25 & 17 & & & \\
\hline${ }^{234} \mathrm{U}$ & 40 & 40 & 3 & 3 & 3 \\
\hline${ }^{235} \mathrm{U}$ & 8 & $<1$ & 1 & & \\
\hline${ }^{236} \mathrm{U}$ & Major $^{b}$ & 425 & 35 & 85 & 70 \\
\hline${ }^{238} \mathrm{U}$ & 34 & 25 & 1 & 0.3 & 0.4 \\
\hline${ }^{238} \mathrm{Pu}$ & 200 & 50 & & & 4000 \\
\hline${ }^{239} \mathrm{Pu}$ & 10 & 1 & $<1$ & $<1$ & 2 \\
\hline${ }^{240} \mathrm{Pu}^{c, d}$ & 6200 & 440 & & & \\
\hline${ }^{241} \mathrm{Pu}$ & 26 & 88 & & & 9 \\
\hline${ }^{244} \mathrm{Pu}$ & 88 & 26 & & & 18 \\
\hline${ }^{241} \mathrm{Am}^{c}$ & 1000 & 100 & & 10 & 30 \\
\hline${ }^{243} \mathrm{Am}$ & 20 & & & & $<5$ \\
\hline${ }^{243} \mathrm{Cm}^{e}$ & 500 & & & & \\
\hline${ }^{244} \mathrm{Cm}^{c}$ & 10 & 5 & 5 & 0.03 & 0.1 \\
\hline${ }^{246} \mathrm{Cm}$ & 700 & 30 & 30 & & \\
\hline${ }^{248} \mathrm{Cm}$ & 70 & 10 & & 1 & 200 \\
\hline
\end{tabular}

${ }^{a}$ From Walker et al., Ref. 3.

${ }^{b}$ More than $5 \%$ of the sample.

${ }^{c}$ For both samples of this isotope.

${ }^{d}$ Also Ta of 350 .

${ }^{e}$ Also $\mathrm{Nd}$ of 20,000 . 
values of cobalt in the original samples. Some of the given impurity values of zinc in the samples appear to be substantially large enough to account for the observed ${ }^{65} \mathrm{Zn}$, but again there appears to be little correlation between the SSMS-derived element zinc and the observed ${ }^{65} \mathrm{Zn}$. Indeed, for ${ }^{238} \mathrm{Pu}$ the SSMS method gives a very large zinc impurity, which should correspond to a ${ }^{65} \mathrm{Zn}$ yield at least a hundred times larger than observed!

Of some concern was the possibility that impurity zirconium could compromise our measurements of the fission product ${ }^{95} \mathrm{Zr}$. The SSMS-deduced amounts of elemental zirconium are given in Table 6. Using an effective $\sigma(n, \gamma)$ for ${ }^{94} \mathrm{Zr}$ of $0.018 \mathrm{~b}$ taken from the Appendix of Ref. 2 results in calculated estimates of ${ }^{95} \mathrm{Zr}$ from ${ }^{94} \mathrm{Zr}(n, \gamma)$ reactions for the samples ${ }^{231} \mathrm{~Pa}$, ${ }^{236} \mathrm{U}$, and ${ }^{246} \mathrm{Cm}$ to be at least a factor of a thousand smaller than observed. Hence, it appears that impurity zirconium in these samples does not measurably contribute to observed ${ }^{95} \mathrm{Zr}$ yields.

Another fission product that was of interest was ${ }^{154} \mathrm{Eu}$, a shielded radioisotope, and therefore expected to have small yields. Of the principal gamma rays expected following decay of ${ }^{154} \mathrm{Eu}$, the low-energy $E_{\gamma}=123.1-\mathrm{keV}$ gamma ray was never observed among the rather substantial Compton background in this region of detected gamma-ray energy (see, e.g. Fig. 6). The next-most intense gamma ray from decay of ${ }^{154} \mathrm{Eu}$ has $E_{\gamma}=1274 \mathrm{keV}$, a value degenerate with a gamma ray following decay of ${ }^{182} \mathrm{Ta}$ as well as with the principal gamma ray following decay of ${ }^{22} \mathrm{Na}$. These background gamma rays interfered sufficiently with data analyses to render unreliable tentatively deduced ${ }^{154} \mathrm{Eu}$ contributions to our measured spectra.

Since it seemed evident that the observed ${ }^{182} \mathrm{Ta}$ decay could not be accounted for by SSMS-deduced impurities in the samples, we looked for another source. It appears from Table 1 that, despite the high purity of the vanadium of the sample container, there was sufficient $\mathrm{Ta}$ impurity to account for even the largest amounts of observed ${ }^{182} \mathrm{Ta}$, although the mechanism by which the ${ }^{182} \mathrm{Ta}$ was transferred from the capsule to the oxide sample is unclear.

\section{RESULTS AND ANALYSES}

\subsection{ANALYSIS OF ${ }^{137}$ Cs ABSOLUTE YIELDS}

One of the empirically observed features of nearly all low-energy neutron-induced fission is the resulting bimodal mass distribution, that is, the fissioning process very strongly favors unequal masses for the two fission fragments. What one obtains then, following measurements of many fissions of the same initial system, is a distribution of the resulting fragment masses into two groups, one designated as the light-mass group and the other as the heavy mass group. Although many details of these mass groups are subject material for ongoing experiments, some general characteristics are known. For example, one may obtain the average mass of the light-mass group, $M_{L}$, and the average mass of the heavy-mass group, $M_{H}$. If $M_{A}$ is the mass of the sample actinide and $M_{n}$ is the mass of the neutron, then mass conservation dictates 


$$
M_{n}+M_{A}=M_{L}+M_{H}+\nu M_{n},
$$

where $\nu$ is the average number of neutrons generated by the fission process.

The present study includes samples having $M_{A}$ between 230 and 248 , and so one aspect concerns the dependences of $M_{L}, M_{H}$, and $\nu$ on $M_{A}$. Data exist which show that $\nu$ varies slowly and somewhat erratically with increasing $M_{A}$, from a value of $\sim 2$ to a value of $\sim 4$. Thus, most of the increase in sample mass must be reflected in increasing $M_{L}+M_{H}$. Interestingly, there are now sufficient data to deduce that nearly all of this increase occurs in $M_{L}$. That is, for neutron fission of the various actinides (on our list), the heavy-mass distribution changes only moderately and $M_{H}(\sim 140)$ changes very little for $M_{A}$ between 230 and 248. One may speculate that the physics being observed is related to shell-model aspects of the description of nuclear isotopes in this mass region; however, such discussion is outside the scope of this report. We point out that the phenomenon has been experimentally observed, and that we will utilize this observation to aid in the presentation and understanding of our data.

Of the various fission products studied (see Table 3), the isotope ${ }^{137} \mathrm{Cs}$ provided the best results to compare with "expectations." Decay of this radionuclide is readily characterized by measurement of a single gamma ray having energy $E_{\gamma}=661.6 \mathrm{keV}$ (which, to be accurate, is due to decay of the isomeric state of the daughter ${ }^{13} \mathrm{Ba}$ radionuclide). This gamma ray happens to be well separated in energy from essentially all other gamma rays we are likely to observe in the present experiment (see, e.g., Fig. 6). Furthermore, the half life of ${ }^{137} \mathrm{Cs}$ is sufficiently long compared to the time scales of the present experiment that details of the irradiation history and times of measurements have essentially no effect on the overall results. Lastly, the mass of this radionuclide is close to $M_{H}$ for all of the actinide samples studied, as so the yield of ${ }^{137} \mathrm{Cs}$ is expected to be (relatively) large and (relatively, again) constant as one progresses from $M_{A}=230$ to 248 , at least to a first approximation.

The yield of any fission product, $Y$, should be determined from the experiment as follows:

$$
Y=N_{s} \sigma_{f} F N_{n}
$$

where $N_{s}$ is the number of sample nuclei, $\sigma_{f}$ is the cross section for fission, $F$ is the fraction of the fissions which result in the desired fission product, and $N_{n}$ is the total neutron fluence through the sample. We now discuss these experimental parameters in reverse order.

We have, according to Broadhead et al., ${ }^{2}$ some information on the neutron flux of the reactor used for this experiment. The mid-plane full-power flux value is given (page 2 of Ref. 2) as $5 \times 10^{15}$ neutrons $/ \mathrm{cm}^{2} / \mathrm{s}$; the variation of flux with respect to distance from the midplane is given in Fig. 2 of Ref. 2; and the position of the physics specimens fuel pin is given on page 21 of Ref. 2. Initially, it was anticipated that the samples would be exposed to a total neutron fluence equivalent to 90 days at full power, and all of the preirradiation analyses ${ }^{2}$ were based upon that value. The actual irradiation history will be discussed in the next section. It was equivalent to a total of $\sim 63$ days at full power spread out over a year between August 24, 1982, and August 31, 1983. With the information available, one may deduce that $N_{n}$ varied between $1.6 \times 10^{22}$ nertrons and $2.6 \times 10^{22}$ neutrons, depending upon the position of the sample actinide in the rod. 
The fraction of fissions, $F$, producing the radionuclide ${ }^{137} \mathrm{Cs}$ has been evaluated for fastneutron induced fission for 13 of our actinide materials by Rider, ${ }^{8}$ with values of $F$ lying between 0.0582 and 0.0714 and with associated uncertainties between 0.5 and $23 \%$ of $F$. For the sample actinides on our list but not included in Rider's evaluation, ${ }^{8}$ we used a value of $F$ $=0.064$ and an associated uncertainty of $10 \%$ of $F$. The actinides for which this value of $F$ was used include ${ }^{230} \mathrm{Th},{ }^{244} \mathrm{Pu}$, and the four curium isotopes.

The fission cross section, $\sigma_{f}$, depends upon the incident-neutron energy, and as shown on page 3 of Ref. 2, the Dounreay PFR neutron-energy spectrum extends from below $1 \mathrm{keV}$ to well above $1 \mathrm{MeV}$. To somewhat simplify the preirradiation analyses, Broadhead et al. ${ }^{2}$ deduced an "average" $\sigma_{f}$, which (values of $\sigma_{f}$ ) are given in Table 20 of that report. ${ }^{2}$ We used the values of $\sigma_{f}$ thus given for our samples between ${ }^{230} \mathrm{Th}$ and ${ }^{246} \mathrm{Cm}$, and (since no value was given in the referenced table) the value of $\sigma_{f}$ for ${ }^{248} \mathrm{Cm}$ as given in the appendix of that report $^{2}$ was used.

For many of the samples, the number of sample nuclei, $N_{s}$, is just the number of nuclei of the principal actinide and can be deduced from the data of Table 2 (of this report). However, for several of the samples, the principal actinide makes up only a fraction of the total of the fissioning nuclei in the sample. In several of the samples, initially there were other fissioning nuclei in the sample; for several of the samples other fissioning nuclei "grew" into the sample during the irradiation, and in some cases these "other" nuclei had larger $\sigma_{f}$ than the principal actinide in the sample. The largest addition to $N_{s}$ due to "other" nuclei was for the ${ }^{236} \mathrm{U}$ sample, for which there were almost twice as many fissions of ${ }^{235} \mathrm{U}$ as there were for the principal actinide, ${ }^{236} \mathrm{U}$. The largest contribution to $N_{s}$ due to fissioning of a "grown in" actinide was for the ${ }^{232} \mathrm{Th}$ sample. Broadhead et al. ${ }^{2}$ calculated a growth of $0.225 \mathrm{mg}$ of ${ }^{233} \mathrm{U}$ (from ${ }^{232} \mathrm{Th}$ $+n$ capture, and subsequent decay of $22-\mathrm{min}{ }^{233} \mathrm{Th}$ ) during a 90 -day full-power irradiation. Correcting this value to the actual 63-day full-power irradiation history yields $\sim 0.158 \mathrm{mg}$ of produced ${ }^{233} \mathrm{U}$. To be exact, one ought to compute the effect on $N_{s}$ by using the actual irradiation history, but for our purpose it was assumed that there was an "average" amount of ${ }^{233} \mathrm{U}$ for the entire irradiation, an amount equal to $0.079 \mathrm{mg}$, or $\sim 0.0044$ of the total sample mass. However, comparing $\sigma_{f}$ for ${ }^{233} \mathrm{U}$ with that for ${ }^{232} \mathrm{Th}$ given in Table 20 of Ref. 2 shows that the former is 292 times larger. Hence, to the approximations of the estimations there should have been about 1.3 fissions of ${ }^{233} \mathrm{U}$ for every fission of the ${ }^{232} \mathrm{Th}$ principal actinide. It is difficult to assign an uncertainty to this estimation of the ${ }^{233} \mathrm{U}$ contribution; for purposes of comparison with experimental data we arbitrarily assigned $10 \%$ as the uncertainty.

In this manner, a computation of $Y\left({ }^{137} \mathrm{Cs}\right)$ was carried out for each of our samples. These were then compared with the experimental data given in Table 4. Ratios of the experimental data divided by the computed values are plotted in Fig. 10, where the ratios are plotted as a function of principal sample mass with specific identification given on the figure only when there were several samples having the same mass. Uncertainties associated with the plotted ratios were deduced from uncertainties on $F$ as given in the evaluation ${ }^{8}$ (or else the assigned $10 \%$ to those not in the evaluation), uncertainties on $N_{s}$ due to including "other" contributions (but not due to any uncertainty on the given mass of the principal actinide), and uncertainties on the present experimental data of Table 4, including estimates of the variances on the mean values deduced from the experimental data. Not included are possible uncertainties on $\sigma_{f}$ or $N_{n}$, on the former because there isn't enough information to deduce what they are, and on the latter because any error translates into an overall normalization error which may (anyway) be deduced from the ratios of the data to computation. 
ORNL - DWG 85-13285

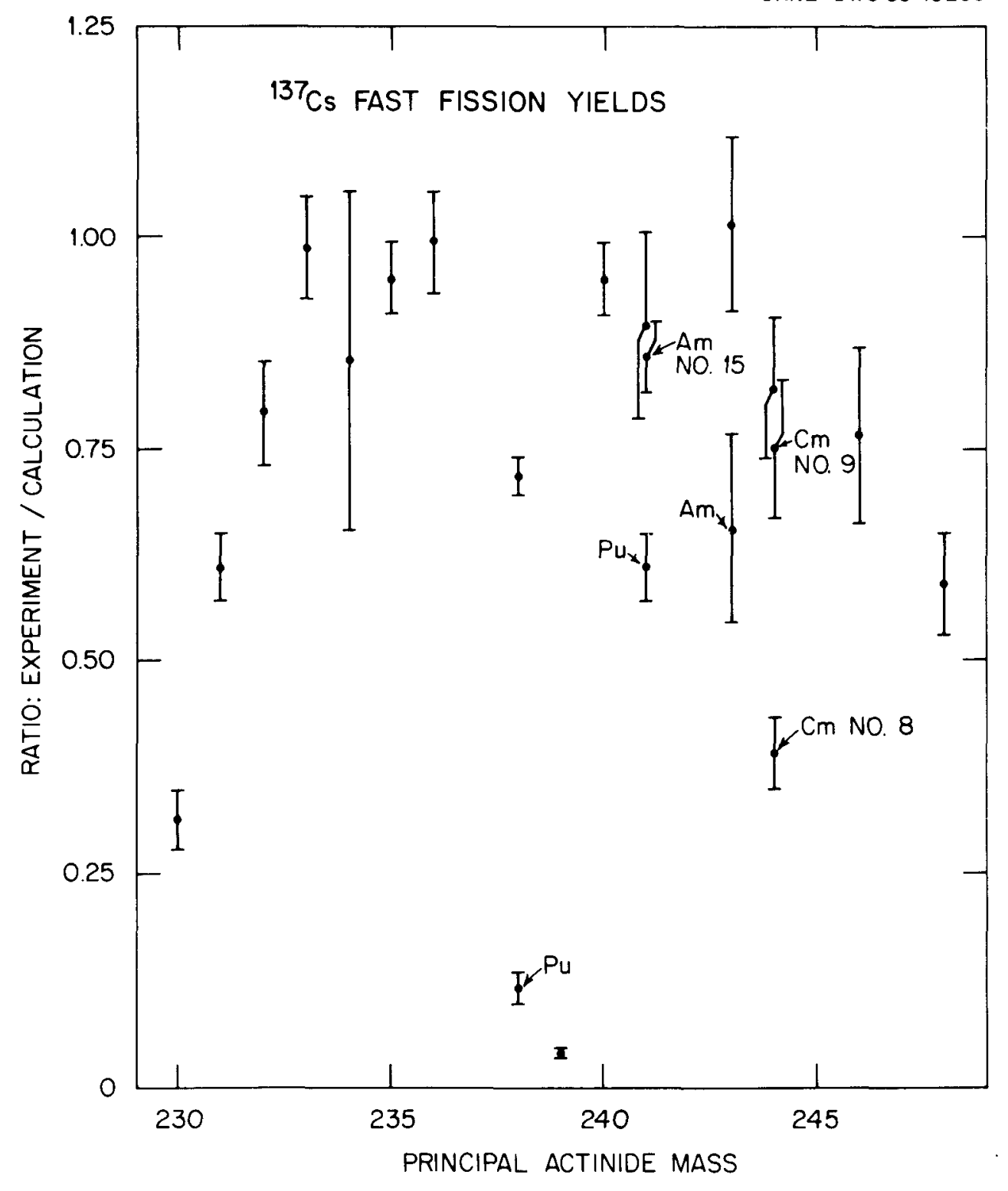

Fig. 10. Absolute yields of ${ }^{137} \mathrm{Cs}$ following fast-neutron fission of 19 different actinides between ${ }^{230} \mathrm{Th}$ and ${ }^{248} \mathrm{Cm}$. The present measurements are plotted as ratios to the calculated yields. The latter were deduced from the sample masses, fission cross sections, ${ }^{137} \mathrm{Cs}$ fission-product yields, and total neutron fluences, which (parameters) were different for the different actinides. As explained in the text, the spectral data were carefully studied in an attempt to deduce plausible explanations for those ratios clearly at variance with the desired value of 1.0 . Several samples with ratio values less than 0.5 may have been damaged when the sample material was removed from the vanadium capsules prior to the preparation of our aliquots. 
What do the results shown in Fig. 10 indicate? First, we cannot believe that the substantial variations observed can be due solely to the gamma-ray-assay portion of the experiment (i.e., the portion of the experiment reported herein). The data were obtained in a very regular manner with proven reliable equipment and measurement techniques. Even so, once we became aware that agreement with computed yields was less than satisfactory, the experiment was completely reviewed for possible, previously undetected substantial sources of error. None were found. Then we reviewed the histories of our samples. As mentioned in Table 2, four samples were possibly damaged during their removal from the vanadium capsules. These samples were ${ }^{233} \mathrm{U},{ }^{238} \mathrm{Pu},{ }^{244} \mathrm{Pu}$, and ${ }^{244} \mathrm{Cm}(\# 8)$. The results observed for the last three named samples may be related to the damage; that is, there was a loss of sample material. For the other samples there was no ready explanation for observed disagreements.

Of the four parameters of Eq. (1), $N_{s}, \sigma_{f}, F$, and $N_{n}$, the only one amenable to be checked by the present experiment is $N_{s}$, and that one for only a portion of the principal actinides. As for the total neutron fluence, $N_{n}$, the data in Fig. 10 might be interpreted to suggest a total neutron fluence of, perhaps, 5 to $10 \%$ less than deduced above, but a larger decrement seems unlikely. As for the "one-group" fission cross section, $\sigma_{f}$, given in Table 20 of Ref. 2, the given values may need to be reconsidered. As for the fission-product yield, $F$ for ${ }^{137} \mathrm{Cs}$, there may well be very moderate adjustments to the values in the evaluation ${ }^{8}$ and one may quarrel with the assumed $F=0.064$ for the actinides not as yet in the evaluation. However, as discussed above, it seems unlikely that any of the values of $F$ used in the computation are in error by as much as $20 \%$, except perhaps for ${ }^{230} \mathrm{Th}$ and ${ }^{248} \mathrm{Cm}$, and an error of $20 \%$ is insufficient to account for the departure from unity of several ratios in Fig. 10.

So, the one parameter that could be checked independently by the present experiment is $N_{s}$, and this we have done for those actinides having sufficient data on one, or more, observable gamma rays which could be ascribed as due to decay of the desired principal actinide. The results of these studies apply to the determinations of $N_{s}$ only for the amount of material in our samples.

\subsection{1 ${ }^{230}$ Th Sample}

The sample labelled ${ }^{2233} \mathrm{U}$ " but believed to be ${ }^{230} \mathrm{Th}$ because of the observed ${ }^{226} \mathrm{Ra}$ decay was studied for three days with the LEPS detector using $D$ (of Fig. 1) $=5 \mathrm{~cm}$. The most intense gamma ray from decay of ${ }^{230} \mathrm{Th}$ has $E_{\gamma}=67.73 \mathrm{keV}$, which is accidently degenerate with the most intense gamma ray following decay of ${ }^{182} \mathrm{Ta}$. The next most intense gamma ray following decay of ${ }^{230} \mathrm{Th}$ has $E_{\gamma}=143.6 \mathrm{keV}$ and a branching ratio ${ }^{4}$ of $0.044 \%$ with an uncertainty of $\sim 9 \%$ its value. Analysis of the results for the observed peak corresponding to $E_{\gamma}=143.6 \mathrm{keV}$ yielded a mass of $(1.07 \pm 0.10) \times 10^{-4} \mathrm{~g}$. This value may be compared with an expected mass of $2.79 \times 10^{-4} \mathrm{~g}$ at the beginning of the irradiation. Using the gamma-ray-assay deduced value of the ${ }^{230} \mathrm{Th}$ mass would result in a ${ }^{137} \mathrm{Cs}$ experimental/calculated ratio of $0.815 \pm 0.110$, a ratio closer to unity than exhibited on Fig. 10 . 


\subsection{2 ${ }^{231}$ Pa Sample}

The strongest transition following decay of ${ }^{231} \mathrm{~Pa}$ has $E_{\gamma}=27.4 \mathrm{keV}$ and a branching ratio of $-9.3 \%$. This branching ratio, however, has an uncertainty of $\sim 20 \%$ of its value. ${ }^{4}$ There is a definite peak corresponding to $E_{\gamma}=27.4 \mathrm{keV}$ observed in the LEPS data for ${ }^{231} \mathrm{~Pa}$. Unfortunately, a peak is observed at this energy in every LEPS spectrum, e.g., Fig. 2, which is ascribed to detection of tellurium $\mathrm{x}$ rays. An estimate was made of the contribution to the peak corresponding to $E_{\gamma}=27.4 \mathrm{keV}$ in the ${ }^{231} \mathrm{~Pa}$ LEPS spectra due to detection of tellurium $\mathrm{x}$ rays under the assumption that these $\mathrm{x}$ rays were due to decay of ${ }^{125} \mathrm{Sb}$. After subtracting this estimate, the remainder of the yield of the peak corresponding to $E_{\gamma}=$ $27.4 \mathrm{keV}$ was used to deduce a mass for the ${ }^{231} \mathrm{~Pa}$ contribution. The result was a mass of (3.1 $\pm 0.8) \times 10^{-4} \mathrm{~g}$, which may be compared with $3.46 \times 10^{-4} \mathrm{~g}$ at the beginning of the irradiation or with $-3.25 \times 10^{-4} \mathrm{~g}$ estimated at the end of the irradiation.

\subsection{3 ${ }^{238}$ Pu Sample}

The half life of this actinide is short enough so that the principal gamma-ray transition at $E_{\gamma}=43.49 \mathrm{keV}$ is observed despite its small branching ratio. The branching ratio has an uncertainty of $\sim 3 \%$ of its value. ${ }^{4}$ The two gamma-ray-assay measurements with the LEPS detector resulted in mass determinations of $(3.64 \pm 0.14) \times 10^{-5} \mathrm{~g}$ and $(3.53 \pm 0.14) \times$ $10^{-5} \mathrm{~g}$, for an average of $(3.58 \pm 0.12) \times 10^{-5} \mathrm{~g}$. This gamma-ray-assay deduced value compares with expected values (see Table 2) of $3.49 \times 10^{-4} \mathrm{~g}$ at the beginning of the irradiation or $-3.34 \times 10^{-4} \mathrm{~g}$ at the end of the irradiation. This sample was labelled as damaged. Apparently some $90 \%$ of this sample was "lost" prior to preparation of our aliquot. Using the gamma-ray-assay deduced mass value results in a ratio of experimental ${ }^{137} \mathrm{Cs}$ yield to calculated yield of $1.13 \pm 0.19$, where the uncertainty is due almost entirely to that assigned ${ }^{8}$ to the evaluated value of $F$.

\subsection{4 ${ }^{239}$ Pu Sample}

Although the half life of this actinide is rather long and the branching ratio of the principal gamma ray is quite small, the energy of this gamma ray, $E_{\gamma}=51.52 \mathrm{keV}$, is favorable in the LEPS spectrum by being sufficiently different from other observed gamma rays. A measurement over $\sim 3$ days was made in which $D$ (of Fig. 1) was set at $5 \mathrm{~cm}$. A very small but well-defined peak was observed at the correct energy. A manual analysis of this peak resulted in a net peak yield of $\sim 9000$ counts out of $1.7 \times 10^{6}$ gross counts, with a statistical error of $-30 \%$ on the net yield. Taking all of the corrections into consideration results in a mass of $(2.6 \pm 0.8) \times 10^{-5} \mathrm{~g}$ for the principal actinide. This value is substantially smaller than the $7.99 \times 10^{-4} \mathrm{~g}$ anticipated at the beginning of the irradiation or $-7.56 \times 10^{-4} \mathrm{~g}$ estimated at the end of the irradiation. More than $96 \%$ of the sample was lost prior to preparation of our aliquot, but we cannot deduce when such loss may have occurred. The ratio of measured ${ }^{137} \mathrm{Cs}$ yield calculated becomes $1.22 \pm 0.37$ using the gamma-ray-assay deduced mass.

\subsection{5 ${ }^{240}$ Pu Sample}

The principal gamma ray associated with decay of this actinide has an energy $E_{\gamma}=$ $45.24 \mathrm{keV}$ and is essentially degenerate with the $E_{\gamma}=45.30 \mathrm{keV}$ gamma ray due to decay of ${ }^{155} \mathrm{Eu}$. The yield of the observed peak corresponding to detection of these two gamma rays is 
too large for just ${ }^{155} \mathrm{Eu}$ decay. The data were analyzed to ascertain if a mass of either of the ${ }^{240} \mathrm{Pu}$ samples could be deduced after accounting for the ${ }^{155} \mathrm{Eu}$ contribution. The uncertainty in this procedure is rather large, and the best that could be determined was that the data were consistent with expected ${ }^{240} \mathrm{Pu}$ masses determined from the data given in Table 2.

\subsection{6 ${ }^{241}$ Pu Sample}

This sample clearly contains much more ${ }^{241} \mathrm{Am}$ than would be deduced from the data tabulated in Table 24 of Broadhead et al. ${ }^{2}$ Our gamma-ray-assay data indicate $>6$ times as much ${ }^{241} \mathrm{Am}$ as tabulated. ${ }^{2}$ The data were studied to try to determine the amount of ${ }^{241} \mathrm{Pu}$ in the sample. The principal gamma ray due to decay of ${ }^{241} \mathrm{Pu}$ has $E_{\gamma}=148.6 \mathrm{keV}$, a convenient energy, but a rather small branching ratio, ${ }^{4} I_{\gamma}=1.9 \times 10^{-6}$. A gamma-ray measurement was made for $\sim 27$ hours for which $D$ of Fig. 1 was $10 \mathrm{~cm}$. This measurement was analyzed to provide a mass for the ${ }^{241} \mathrm{Pu}$ content of this sample. We obtained a mass of $5.03 \times 10^{-5} \mathrm{~g}$ with an uncertainty of $3.8 \%$ on this value. This value of mass may be compared with $8.20 \times$ $10^{-5} \mathrm{~g}$ at the beginning of the irradiation according to the data of Table 2 . Using the gamma-ray-assay mass value for ${ }^{241} \mathrm{Pu}$ results in a ${ }^{137} \mathrm{Cs}$ ratio value of $1.00 \pm 0.07$.

\subsection{7 ${ }^{241}$ Am Sample}

This actinide is probably the easiest actinide to obtain a precision mass value by gammaray assay. Our data indicate mass values of $(9.8 \pm 0.3) \times 10^{-6} \mathrm{~g}$ for sample \#14 and $(9.15$ $\pm 0.28) \times 10^{-5} \mathrm{~g}$ for sample \#15, where the uncertainties include those associated with the efficiency calibration of the LEPS detector. These mass values may be compared with the expected mass values of $10.4 \times 10^{-6} \mathrm{~g}$ for sample \#14 and $9.55 \times 10^{-5} \mathrm{~g}$ for sample \#15 at the beginning of the irradiation, and with estimated mass values of $9.83 \times 10^{-6} \mathrm{~g}$ for sample $\# 14$ and $9.05 \times 10^{-5} \mathrm{~g}$ for sample \#15 at the end of the irradiation.

\subsection{8 ${ }^{243}$ Am Sample}

Determining a mass value for this actinide requires a little more care than determining a value for ${ }^{241} \mathrm{Am}$. Decay of ${ }^{243} \mathrm{Am}$ results in a number of gamma-ray transitions, many of which are the same transitions observed in the decay of ${ }^{243} \mathrm{Cm}$. The major difference is a strong $E_{\gamma}=74.66-\mathrm{keV}$ transition gamma ray observed in the decay of ${ }^{243} \mathrm{Am}$, which is absent in the decay of ${ }^{243} \mathrm{Cm}$. The $74.66-\mathrm{keV}$ gamma ray, however, is essentially degenerate with the $\mathrm{Pb} K_{\alpha 1} \times$ ray observed as a part of the background, and so small corrections were needed to account for this contaminant. We obtained a mass value of $(1.67 \pm 0.07) \times 10^{-5} \mathrm{~g}$ for the ${ }^{243} \mathrm{Am}$ in the sample. This value is somewhat smaller than the value of $1.96 \times 10^{-5} \mathrm{~g}$ expected at the beginning of the irradiation or the value of $1.89 \times 10^{-5} \mathrm{~g}$ estimated for the end of the irradiation. Using the gamma-ray-assay value of the ${ }^{243} \mathrm{Am}$ mass would result in a ${ }^{137} \mathrm{Cs}$ ratio of $0.77 \pm 0.13$, where the uncertainty is dominated by the uncertainty assigned ${ }^{8}$ to the evaluated value of $F$.

\subsection{9 ${ }^{243} \mathrm{Cm}$ Sample}

The mass of this sample had to be comparatively small because of the activity associated with the principal actinide. Our gamma-ray-assay data indicated a mass value of (3.31 \pm $0.20) \times 10^{-6} \mathrm{~g}$ at the end of the irradiation. This value may be compared with $3.34 \times$ $10^{-6} \mathrm{~g}$ expected at the beginning of the irradiation according to the data in Table 2 , and with $-3.10 \times 10^{-6} \mathrm{~g}$ estimated at the end of the irradiation. 


\subsubsection{0 ${ }^{244} \mathrm{Cm}$ Sample}

The primary gamma ray due to decay of this actinide has $E_{\gamma}=42.82 \mathrm{keV}$ and is essentially degenerate with $\mathrm{Gd} K_{\alpha} \times$ rays observed from the decay of ${ }^{155} \mathrm{Eu}$. In our spectra the $\mathrm{x}$ ray from the ${ }^{155} \mathrm{Eu}$ decay contributed $-10 \%$ of the peak, and so the two ${ }^{244} \mathrm{Cm}$ mass determinations were only moderately affected. Our gamma-ray-assay data indicated mass values of $(3.02 \pm 0.21) \times 10^{-5} \mathrm{~g}$ and $(1.252 \pm 0.051) \times 10^{-4} \mathrm{~g}$ for our samples \#8 and \#9, respectively. These mass values may be compared with $7.79 \times 10^{-5} \mathrm{~g}$ and $1.59 \pm 10^{-4} \mathrm{~g}$ for samples \#8 and \#9, respectively, at the beginning of the irradiation. Sample \#8 was labelled as damaged when we received our aliquot; the loss of sample may have occurred during this last phase of preparation. We cannot account for the difference in mass for sample \#9. Using the gamma-ray-assay masses would result in ${ }^{137} \mathrm{Cs}$ ratios of $1.01 \pm 0.12$ and $0.95 \pm 0.10$ for samples \#8 and \#9, respectively.

\subsubsection{Other Samples}

Mass determinations could not be made for the actinides not discussed because a definitive gamma-ray transition was not unambiguously observed. However, some conclusions can be drawn from those actinide mass determinations which were made. Of the 13 samples for which the gamma-ray-assay data yielded principal actinide mass values, seven of the gammaray-assay values disagree with mass values expected on the basis of prior reports and assigned aliquots as delineated in Table 2. Of these seven, two may have incurred losses when the samples were removed from the vanadium capsules, namely for the ${ }^{238} \mathrm{Pu}$ and ${ }^{244} \mathrm{Cm} \# 8 \mathrm{sam}$ ples. The lack of agreement for the other five samples is very disturbing and may give one pause before uncritically accepting as valid mass values for the nine actinides for which the gamma-ray-assay data were insufficient to produce principal actinide mass values to check those expected from the data in Table 2. We grant, on the one hand, that the ratios deduced and exhibited in Fig. 10 for ${ }^{137} \mathrm{Cs}$ are by themselves insufficient to specify errors (or their sources) in the masses of our samples, but, on the other hand, accepting the validity of this assertion with regard to data for ${ }^{137} \mathrm{Cs}$ means that the assertion must be valid with regard to data for the other observed fission products. One must accept, perforce, that absolute determinations of fission-product yields from the present gamma-ray-assay data would not be reliable. That is, irrespective of one's bias with regard to any part (or all) of the results discussed in this section, the only acceptable presentation of the data for deduced yields of fission products is as relative yields, and in the next section we choose to present the remainder of our data as yields relative to ${ }^{137} \mathrm{Cs}$ yields.

\subsection{OTHER FISSION-PRODUCT YIELDS}

In this section, the data for other fission products are presented. These other fission products include ${ }^{91} \mathrm{Y},{ }^{95} \mathrm{Zr},{ }^{95} \mathrm{Nb},{ }^{103} \mathrm{Ru},{ }^{106} \mathrm{Ru}-{ }^{106} \mathrm{Rh},{ }^{110 \mathrm{~m}} \mathrm{Ag},{ }^{125} \mathrm{Sb},{ }^{134} \mathrm{Cs},{ }^{141} \mathrm{Ce},{ }^{144} \mathrm{Ce}-{ }^{144} \mathrm{Pr}$, and ${ }^{155} \mathrm{Eu}$. Of the fission products that we observed for all our principal actinides, only ${ }^{95} \mathrm{Nb}$ had to be considered separately because of the long lifetime of its parent, ${ }^{95} \mathrm{Zr}$.

For all of the other fission products the analyses of the spectral data provided results in the form of the number of atoms of radionuclide that were as measured and then were corrected to account for that particular radionuclide decay during the cooling period between the end of the irradiation and the beginning of the gamma-ray-assay counting period. In this manner the 
effective cumulative yield for each radionuclide and for a particular sample was deduced. There are errors in this procedure, which depend upon the half life of the parent of the particular radionuclide being reported, but these errors are quite small compared to other uncertainties of the analyses.

The question of how to interpret these results was next addressed. Clearly, these results were sample dependent, and as such would be most amenable to comparison with calculations such as those done by Broadhead et al. ${ }^{2}$ for the preanalysis, provided the sample characteristics were known. However, as discussed in the last section on the ${ }^{137}$ Cs yields, the evidence does not favor uncritical acceptance of premeasurement designations of our sample aliquots; at least that is our conclusion. Therefore, the decision was made to report deduced radionuclide yields as ratios to the ${ }^{137} \mathrm{Cs}$ yield for each sample that we studied. We did not make corrections in these ratio results to account for fissions by other than the principal actinide, and so for data reported for several of the samples, the reported ratios may be at some variance with values that may be determined for fast-neutron fission of just the principal actinide. As described, however, these ratio results should be amenable to direct comparisons with calculations as described by Broadhead $e t$ al. ${ }^{2}$ and yet also serve as a basis for determining basicphysics fission-product yields for inclusion in future compilations and evaluations of the type performed by Rider. ${ }^{8}$

The deduced ratio results as obtained for all of the identified fission products except ${ }^{95} \mathrm{Nb}$ are given in Table 7. The results for ${ }^{95} \mathrm{Nb}$ are collected separately in Table 8, where the ratio data are given for each separate measurement and are computed as measured; that is, they are effective at the time of the measurement. As mentioned above, the results are given for the sample with no attempt to "correct" the data for contributions by the other-than-principal actinides. Computation of such contributions requires not only a sophisticated isotope inventory computer code such as that used by Broadhead et al. ${ }^{2}$ but also requires accurate nuclear data (cross sections, fission-product yields, decay constants, etc.) and some of these data may be inaccurate or even nonexistent. Indeed, one goal of the present study is to provide data for the testing of such data libraries as are current, and, perhaps, even to provide results leading to direct determination of new fission-product yields.

Uncertainties assigned to the ratio data given in Tables 7 and 8 include uncertainties assigned to gamma-ray branching ratios (see Table 3) and uncertainties associated with the data reduction, including those associated with detector efficiency calibrations and sample gamma-ray attenuation calculations. Measurement uncertainties for ${ }^{137} \mathrm{Cs}$ were $\leqslant 1 \%$, and there is a fully correlated uncertainty of this magnitude for all of the ratio results for a given principal actinide. One advantage of presenting the data as ratios is the essential elimination of uncertainties associated with other parameters of Eq. (1), namely the sample mass, the fission cross section, and the neutron fluence.

In summary, then, we have obtained a nearly complete data set for 11 radioisotopes created by fast-neutron fission of some 19 different principal fissionable actinides. Data for the samples of seven of these actinides, namely ${ }^{233} \mathrm{U},{ }^{235} \mathrm{U},{ }^{238} \mathrm{Pu},{ }^{240} \mathrm{Pu},{ }^{241} \mathrm{Pu},{ }^{241} \mathrm{Am}$, and ${ }^{243} \mathrm{Am}$, should be representative of the principal nuclide, since our estimates indicate that $\geqslant 95 \%$ of the fissions occurred following neutron interaction with atoms of the principal actinide in the sample. On the other hand, we estimate that for six samples, namely ${ }^{232} \mathrm{Th},{ }^{231} \mathrm{~Pa},{ }^{236} \mathrm{U},{ }^{244} \mathrm{Pu}$, ${ }^{246} \mathrm{Cm}$, and ${ }^{248} \mathrm{Cm}$, fast-neutron fissions with nonprincipal actinides accounted for $>20 \%$ of the 
measured yields. Evidently, the best comparisons for the present data will be full-model calculations of the type already reported by Broadhead et al. ${ }^{2}$ Such calculations are quite sophisticated and beyond the scope of an experimental report such as this. However, the experimental ratio data may also be compared with similar data which can be deduced from the evaluated data $^{8}$ at least to uncover any "large" discrepancies. Such comparisons are well within the scope of an experimental paper, as they may be used to indicate possible experimental problems and thus give some quantitative credence to the reported results.

\subsection{COMPARISONS OF EXPERIMENTAL AND EVALUATED FISSION-PRODUCT YIELDS}

The current evaluation ${ }^{8}$ presents yields for fast-neutron fission of 18 different actinides including 13 of the present principal actinides, namely ${ }^{232} \mathrm{Th},{ }^{231} \mathrm{~Pa},{ }^{233,234,235,236,238} \mathrm{U}$, $238,239,240,241 \mathrm{Pu}$, and ${ }^{241,243} \mathrm{Am}$, and five actinides not among the present principal actinides, namely ${ }^{237,238} \mathrm{~Np},{ }^{237} \mathrm{U},{ }^{242} \mathrm{Pu}$, and ${ }^{242} \mathrm{Cm}$. For each individual fission-product-yield data set, ratio data were obtained for each evaluated yield datum with respect to the evaluated datum for ${ }^{137} \mathrm{Cs}$, and an uncertainty was assigned to each calculated ratio. This assigned uncertainty was determined by using the larger of the fractional uncertainties assigned to the yield datum for the particular fission product or to the yield datum for ${ }^{137} \mathrm{Cs}$. Although such a determination does not result in a "correct" uncertainty for a deduced ratio, it should be very adequate for the illustrative purpose at hand.

The experimental data of Table 7 cannot be compared directly to the evaluated ratio data because the latter are determined from fission-product yields deduced as if from an essentially instantaneous irradiation, whereas the data in Table 7 are from a substantially extended irradiation. The measured fission products of Table 7 all have half lives shorter than that for ${ }^{137} \mathrm{Cs}$, with the shortest half life being 32.5 days for ${ }^{141} \mathrm{Ce}$. For these shorter-lived radioisotopes, the details of the irradiation become important, and it was necessary to determine, at least to first order, the adjustments to the measured yields to deduce yields that would have been observed following a short irradiation. To determine these adjustements required a knowledge of the irradiation history at the PFR. This history was provided as a strip chart spanning the period August 24, 1982 to August 31, 1983. For our purpose, time units of a day were considered to be of sufficient detail, and so we averaged any fluctuations of smaller time durations. The operating history that we used is summarized in Table 9. The "maximum" operating power was stated to be $1 / 3$ of full power; however, we adjusted this value modestly downward so as to provide an integrated neutron fluence of 63 full-power days.

A short computer program was written to determine the adjustments to the measured yields. These adjustments, however, are applicable only for those fission products having essentially instantaneous production (i.e., for which the parent half lives were short with respect to time intervals of the measurements) and for which radionuclide decay was the mode of radionuclide disappearance.

The deduced adjustments are collected in Table 10. To determine experimental ratio data for comparison with the evaluated ratio data requires multiplying data in Table 7 by the appropriate factor given in Table 10. 
Table 7. Fission-product yields for fast-neutron fission. Tabulated data are ratios of the yields of the fission product at the top of the column to the yields of ${ }^{137} \mathrm{Cs}$ as of the end of the irradiation.

\begin{tabular}{|c|c|c|c|c|c|c|}
\hline Sample no. ${ }^{a}$ & $\begin{array}{l}\text { Principal } \\
\text { isotope }\end{array}$ & ${ }^{91} Y^{b}$ & ${ }^{95} \mathrm{Zr}$ & ${ }^{103} \mathrm{Ru}^{b}$ & ${ }^{106} \mathrm{Ru}-{ }^{106} \mathrm{Rh}^{b, c}$ & ${ }^{110 \mathrm{~m}} \mathrm{Ag}$ \\
\hline 31 & ${ }^{230} \mathrm{Th}$ & $0.31 \pm 0.04$ & $0.228 \pm 0.011$ & $0.013 \pm 0.002$ & $0.013 \pm 0.001$ & \\
\hline 25 & ${ }^{232} \mathrm{Th}$ & $0.35 \pm 0.04$ & $0.416 \pm 0.017$ & $0.014 \pm 0.002$ & $0.0059 \pm 0.0007$ & \\
\hline 29 & ${ }^{231} \mathrm{~Pa}$ & $0.19 \pm 0.13$ & $0.312 \pm 0.020$ & $0.020 \pm 0.001$ & $0.020 \pm 0.002$ & \\
\hline 32 & ${ }^{233} \mathrm{U}$ & $0.24 \pm 0.03$ & $0.311 \pm 0.007$ & $0.054 \pm 0.002$ & $0.029 \pm 0.003$ & \\
\hline 27 & ${ }^{234} \mathrm{U}$ & $0.20 \pm 0.02$ & $0.335 \pm 0.008$ & & $0.048 \pm 0.004$ & \\
\hline 28 & ${ }^{235} \mathrm{U}$ & $0.21 \pm 0.02$ & $0.340 \pm 0.010$ & $0.108 \pm 0.004$ & $0.055 \pm 0.005$ & \\
\hline 26 & ${ }^{236} \mathrm{U}$ & $0.23 \pm 0.03$ & $0.340 \pm 0.011$ & $0.117 \pm 0.006$ & $0.069 \pm 0.006$ & \\
\hline 11 & ${ }^{238} \mathrm{U}$ & $0.14 \pm 0.02$ & $0.285 \pm 0.009$ & $0.246 \pm 0.007$ & $0.355 \pm 0.029$ & \\
\hline 30 & ${ }^{238} \mathrm{Pu}$ & $0.092 \pm 0.010$ & $0.245 \pm 0.008$ & $0.159 \pm 0.008$ & $0.321 \pm 0.027$ & \\
\hline 23 & ${ }^{239} \mathrm{Pu}$ & $0.11 \pm 0.05$ & $0.220 \pm 0.006$ & $0.172 \pm 0.009$ & $0.378 \pm 0.032$ & $0.00010 \pm 0.00004$ \\
\hline 22 & ${ }^{240} \mathrm{Pu}$ & $0.082 \pm 0.010$ & $0.225 \pm 0.006$ & $0.040 \pm 0.002$ & $0.120 \pm 0.010$ & $0.00017 \pm 0.00002$ \\
\hline 21 & ${ }^{240} \mathrm{Pu}$ & $0.079 \pm 0.012$ & $0.220 \pm 0.006$ & $0.053 \pm 0.003$ & $0.128 \pm 0.011$ & $0.00015 \pm 0.00002$ \\
\hline 24 & ${ }^{241} \mathrm{Pu}$ & $0.083 \pm 0.010$ & $0.195 \pm 0.005$ & $0.076 \pm 0.004$ & $0.242 \pm 0.021$ & $0.00013 \pm 0.00001$ \\
\hline 20 & ${ }^{244} \mathrm{Pu}$ & $0.052 \pm 0.021$ & $0.161 \pm 0.005$ & $0.176 \pm 0.009$ & $0.615 \pm 0.049$ & $0.00027 \pm 0.00004$ \\
\hline 15 & ${ }^{241} \mathrm{Am}$ & $0.069 \pm 0.044$ & $0.200 \pm 0.008$ & $0.144 \pm 0.006$ & $0.322 \pm 0.028$ & $0.00013 \pm 0.00002$ \\
\hline 14 & ${ }^{241} \mathrm{Am}$ & $0.080 \pm 0.009$ & $0.196 \pm 0.006$ & $0.195 \pm 0.007$ & $0.455 \pm 0.039$ & \\
\hline 12 & ${ }^{243} \mathrm{Am}$ & $0.071 \pm 0.025$ & $0.168 \pm 0.006$ & $0.183 \pm 0.006$ & $0.495 \pm 0.041$ & $0.00020 \pm 0.00002$ \\
\hline 10 & ${ }^{243} \mathrm{Cm}$ & & $0.161 \pm 0.005$ & $0.087 \pm 0.003$ & $0.227 \pm 0.019$ & $0.00027 \pm 0.00003$ \\
\hline 9 & ${ }^{244} \mathrm{Cm}$ & $0.043 \pm 0.009$ & $0.151 \pm 0.005$ & $0.198 \pm 0.007$ & $0.667 \pm 0.056$ & $0.00030 \pm 0.00003$ \\
\hline 8 & ${ }^{244} \mathrm{Cm}$ & $0.069 \pm 0.019$ & $0.147 \pm 0.005$ & $0.159 \pm 0.006$ & $0.431 \pm 0.036$ & $0.00029 \pm 0.00003$ \\
\hline 5 & ${ }^{246} \mathrm{Cm}$ & $0.054 \pm 0.019$ & $0.122 \pm 0.005$ & $0.198 \pm 0.007$ & $0.690 \pm 0.057$ & $0.00040 \pm 0.00003$ \\
\hline 4 & ${ }^{248} \mathrm{Cm}$ & $0.036 \pm 0.017$ & $0.108 \pm 0.004$ & $0.143 \pm 0.006$ & $0.445 \pm 0.037$ & $0.00045 \pm 0.00003$ \\
\hline
\end{tabular}

${ }^{a}$ The tabulated data are for the sample.

${ }^{b}$ Results may be suspect; consult discussion in the text.

${ }^{c}$ Results applicable to first isotope $\left({ }^{106} \mathrm{Ru},{ }^{144} \mathrm{Ce}\right)$ include analyses of gamma radiation from decay of the second isotope. 
Table 7. Cont'd

\begin{tabular}{|c|c|c|c|c|c|c|}
\hline Sample no. ${ }^{a}$ & $\begin{array}{l}\text { Principal } \\
\text { isotope }\end{array}$ & ${ }^{125} \mathrm{Sb}$ & ${ }^{134} \mathrm{Cs}$ & ${ }^{141} \mathrm{Ce}$ & ${ }^{144} \mathrm{Ce}-{ }^{144} \operatorname{Pr}^{c}$ & ${ }^{155} \mathrm{Eu}$ \\
\hline 31 & ${ }^{230} \mathrm{Th}$ & $0.0058 \pm 0.0007$ & $0.0033 \pm 0.0003$ & $0.224 \pm 0.008$ & $0.780 \pm 0.027$ & $0.006 \pm 0.003$ \\
\hline 25 & ${ }^{232} \mathrm{Th}$ & $0.0127 \pm 0.0018$ & $0.0033 \pm 0.0004$ & $0.184 \pm 0.018$ & $0.694 \pm 0.026$ & $0.0022 \pm 0.0007$ \\
\hline 29 & ${ }^{231} \mathrm{~Pa}$ & $0.0154 \pm 0.0036$ & $0.0033 \pm 0.0003$ & $0.214 \pm 0.012$ & $0.545 \pm 0.025$ & $0.0042 \pm 0.0008$ \\
\hline 32 & ${ }^{233} U$ & $0.0191 \pm 0.0015$ & $0.0050 \pm 0.0004$ & $0.188 \pm 0.007$ & $0.464 \pm 0.017$ & $0.0032 \pm 0.0007$ \\
\hline 27 & ${ }^{234} U$ & $0.0126 \pm 0.0009$ & $0.0050 \pm 0.0004$ & $0.210 \pm 0.016$ & $0.538 \pm 0.020$ & \\
\hline 28 & ${ }^{235} \mathrm{U}$ & $0.0054 \pm 0.0006$ & $0.0054 \pm 0.0004$ & $0.188 \pm 0.030$ & $0.605 \pm 0.019$ & $0.0061 \pm 0.0008$ \\
\hline 26 & ${ }^{236} \mathrm{U}$ & $0.0075 \pm 0.0008$ & $0.0056 \pm 0.0005$ & $0.190 \pm 0.018$ & $0.613 \pm 0.024$ & $0.0095 \pm 0.0011$ \\
\hline 11 & ${ }^{238} \mathrm{U}$ & $0.0085 \pm 0.0006$ & $0.0060 \pm 0.0005$ & $0.190 \pm 0.010$ & $0.530 \pm 0.021$ & $0.0212 \pm 0.0022$ \\
\hline 30 & ${ }^{238} \mathrm{Pu}$ & $0.0253 \pm 0.0016$ & $0.0061 \pm 0.0006$ & $0.165 \pm 0.009$ & $0.346 \pm 0.011$ & $0.018 \pm 0.002$ \\
\hline 23 & ${ }^{239} \mathrm{Pu}$ & $0.0172 \pm 0.0012$ & $0.0057 \pm 0.0005$ & $0.147 \pm 0.006$ & $0.352 \pm 0.016$ & $0.021 \pm 0.003$ \\
\hline 22 & ${ }^{240} \mathrm{Pu}$ & $0.0140 \pm 0.0009$ & $0.0056 \pm 0.0005$ & $0.148 \pm 0.006$ & $0.434 \pm 0.014$ & $0.034 \pm 0.005$ \\
\hline 21 & ${ }^{240} \mathrm{Pu}$ & $0.0140 \pm 0.0009$ & $0.0057 \pm 0.0005$ & $0.155 \pm 0.007$ & $0.432 \pm 0.011$ & $0.033 \pm 0.004$ \\
\hline 24 & ${ }^{241} \mathrm{Pu}$ & $0.0091 \pm 0.0008$ & $0.0055 \pm 0.0005$ & $0.172 \pm 0.011$ & $0.452 \pm 0.014$ & $0.043 \pm 0.005$ \\
\hline 20 & ${ }^{244} \mathrm{Pu}$ & $0.0071 \pm 0.0007$ & $0.0047 \pm 0.0004$ & $0.165 \pm 0.006$ & $0.489 \pm 0.014$ & $0.062 \pm 0.010$ \\
\hline 15 & ${ }^{241} \mathrm{Am}$ & $0.033 \pm 0.006$ & $0.0067 \pm 0.0006$ & $0.162 \pm 0.010$ & $0.397 \pm 0.014$ & $0.057 \pm 0.007$ \\
\hline 14 & ${ }^{241} \mathrm{Am}$ & $0.035 \pm 0.006$ & $0.0074 \pm 0.0006$ & $0.172 \pm 0.010$ & $0.394 \pm 0.014$ & $0.057 \pm 0.007$ \\
\hline 12 & ${ }^{243} \mathrm{Am}$ & $0.0227 \pm 0.0017$ & $0.0054 \pm 0.0005$ & $0.178 \pm 0.020$ & $0.450 \pm 0.016$ & $0.093 \pm 0.010$ \\
\hline 10 & ${ }^{243} \mathrm{Cm}$ & $0.0286 \pm 0.0020$ & $0.0073 \pm 0.0007$ & $0.150 \pm 0.008$ & $0.400 \pm 0.018$ & \\
\hline 9 & ${ }^{244} \mathrm{Cm}$ & $0.0237 \pm 0.0023$ & $0.0056 \pm 0.0005$ & $0.180 \pm 0.018$ & $0.438 \pm 0.016$ & $0.078 \pm 0.008$ \\
\hline 8 & ${ }^{244} \mathrm{Cm}$ & $0.0238 \pm 0.0023$ & $0.0060 \pm 0.0006$ & $0.181 \pm 0.015$ & $0.432 \pm 0.016$ & $0.076 \pm 0.008$ \\
\hline 5 & ${ }^{246} \mathrm{Cm}$ & $0.0170 \pm 0.0016$ & $0.0055 \pm 0.0005$ & $0.185 \pm 0.011$ & $0.509 \pm 0.017$ & $0.099 \pm 0.011$ \\
\hline 4 & ${ }^{248} \mathrm{Cm}$ & $0.0133 \pm 0.0009$ & $0.0057 \pm 0.0005$ & $0.208 \pm 0.009$ & $0.578 \pm 0.019$ & $0.109 \pm 0.012$ \\
\hline
\end{tabular}


Table 8. ${ }^{95} \mathrm{Nb}$ fission-product yields for fast-neutron fission. Data are ratios of ${ }^{95} \mathrm{Nb}$ yields to ${ }^{137} \mathrm{Cs}$ yields at the time of the measurement

\begin{tabular}{|c|c|c|c|c|}
\hline $\begin{array}{c}\text { Principal } \\
\text { actinide }\end{array}$ & $\begin{array}{c}\text { Time after } \\
\text { irradiation }^{a}\end{array}$ & $\begin{array}{c}\text { Ratio } \\
\left(\times 10^{-3}\right) \\
\end{array}$ & $\begin{array}{c}\text { Time after } \\
\text { irradiation }^{a}\end{array}$ & $\begin{array}{c}\text { Ratio } \\
\left(\times 10^{-3}\right) \\
\end{array}$ \\
\hline${ }^{230} \mathrm{Th}^{b}$ & 281 & $11.0 \pm 0.2^{c}$ & 435 & $2.37 \pm 0.03$ \\
\hline${ }^{232} \mathrm{Th}$ & 315 & $14.9 \pm 0.3$ & 428 & $4.65 \pm 0.07$ \\
\hline${ }^{231} \mathrm{~Pa}$ & 321 & $10.6 \pm 0.2$ & 434 & $3.17 \pm 0.06$ \\
\hline${ }^{233} \mathrm{U}$ & 316 & $8.04 \pm 0.17$ & 428 & $3.18 \pm 0.05$ \\
\hline${ }^{234} \mathrm{U}$ & 450 & $3.03 \pm 0.05$ & 624 & $0.48 \pm 0.02$ \\
\hline${ }^{235} \mathrm{U}$ & 284 & $16.1 \pm 0.07$ & 446 & $3.24 \pm 0.06$ \\
\hline${ }^{236} U$ & 284 & $14.2 \pm 0.05$ & 436 & $3.35 \pm 0.05$ \\
\hline${ }^{238} \mathrm{U}$ & 284 & $13.6 \pm 0.03$ & 447 & $2.68 \pm 0.04$ \\
\hline${ }^{238} \mathrm{Pu}$ & 316 & $8.37 \pm 0.14$ & 450 & $2.23 \pm 0.04$ \\
\hline${ }^{239} \mathrm{Pu}$ & 321 & $8.00 \pm 0.17$ & 447 & $2.13 \pm 0.05$ \\
\hline${ }^{240} \mathrm{Pu} \# 22$ & 320 & $7.85 \pm 0.15$ & 431 & $2.44 \pm 0.04$ \\
\hline${ }^{240} \mathrm{Pu} \# 21$ & 301 & $9.62 \pm 0.23$ & 434 & $2.41 \pm 0.04$ \\
\hline${ }^{241} \mathrm{Pu}$ & 320 & $6.85 \pm 0.13$ & 432 & $2.15 \pm 0.04$ \\
\hline${ }^{244} \mathrm{Pu}$ & 319 & $5.26 \pm 0.10$ & 446 & $1.50 \pm 0.03$ \\
\hline${ }^{241} \mathrm{Am} \# 15$ & 281 & $9.55 \pm 0.23$ & 448 & $1.87 \pm 0.03$ \\
\hline${ }^{241} \mathrm{Am} \mathrm{\# 14}$ & 282 & $10.95 \pm 0.16$ & 438 & $2.10 \pm 0.04$ \\
\hline${ }^{243} \mathrm{Am}$ & 281 & $9.02 \pm 0.13$ & 440 & $1.70 \pm 0.03$ \\
\hline${ }^{243} \mathrm{Cm}$ & 300 & $6.82 \pm 0.17$ & 449 & $1.41 \pm 0.06$ \\
\hline${ }^{244} \mathrm{Cm} \# 9$ & 295 & $6.99 \pm 0.17$ & 432 & $1.70 \pm 0.04$ \\
\hline${ }^{244} \mathrm{Cm} \# 8$ & 299 & $5.08 \pm 0.11$ & - & - \\
\hline${ }^{246} \mathrm{Cm}$ & 299 & $5.48 \pm 0.09$ & 439 & $1.25 \pm 0.02$ \\
\hline${ }^{248} \mathrm{Cm}$ & 299 & $4.46 \pm 0.08$ & 437 & $1.10 \pm 0.02$ \\
\hline
\end{tabular}

${ }^{a}$ In days.

${ }^{b} \mathrm{~A}$ third measurement, 600 days after the end of the irradiation resulted in a ratio of $(432 \pm 6) \times 10^{-6}$.

'Uncertainties are primarily on peak area determinations. 
Table 9. Irradiation history at the Dounreay PFR for the present experiment

\begin{tabular}{lll}
\hline \multicolumn{1}{c}{ Month } & \multicolumn{1}{c}{$\begin{array}{c}\text { Dates } \\
\text { reactor up }\end{array}$} & \multicolumn{1}{c}{$\begin{array}{c}\text { Dates } \\
\text { reactor down }\end{array}$} \\
\hline $\begin{array}{ll}\text { August, 1982 } \\
\text { September }\end{array}$ & $24-25,27-31$ & 26 \\
$\begin{array}{l}\text { October } \\
\text { November }\end{array}$ & $1-30^{b}$ & \\
December, 1982 & $1-24$ & $25-31$ \\
January, 1983 & & $1-30$ \\
February & $20-22$ & $1-31$ \\
March & $15-26$ & $1-19,23-31$ \\
April & $21-31$ & $1-14,27-28$ \\
May & $1-30$ & $1-20$ \\
June & $1-15$ & $16-31$ \\
July & $14-30$ & $1-13$ \\
August, 1983 & $1-31$ & \\
\hline
\end{tabular}

${ }^{a}$ At $\sim 0.3 \times$ full power of the PFR.

${ }^{b}$ Including three days at $0.1 \times$ full power of the PFR.

Table 10. Adjustment factors for the experimental ratio data. These factors were deduced from the irradiation history of Table 9 and the half life of each fission product so as to provide an estimate of the ratio values that would have been measured following an instantaneous irradiation

\begin{tabular}{ll}
\hline $\begin{array}{c}\text { Fission } \\
\text { product }\end{array}$ & $\begin{array}{c}\text { Adjustment } \\
\text { factor }^{a}\end{array}$ \\
\hline${ }^{91} \mathrm{Y}$ & 3.17 \\
${ }^{95} \mathrm{Zr}$ & 2.98 \\
${ }^{103} \mathrm{Ru}$ & 4.27 \\
${ }^{106} \mathrm{Ru}$ & 1.300 \\
${ }^{125} \mathrm{Sb}$ & 1.102 \\
${ }^{141} \mathrm{Ce}$ & 4.96 \\
${ }^{144} \mathrm{Ce}$ & 1.404 \\
${ }^{155} \mathrm{Eu}$ & 1.052 \\
\hline
\end{tabular}

${ }^{a}$ Uncertainty estimated at \pm 4 in units of the last digit. 
The "evaluated" ratio data and associated uncertainties are exhibited in Figs. 11 through 18 compared with adjusted experimental data for eight observed fission products. The adjusted experimental data are exhibited as solid points, and the ratios deduced from the evaluation are exhibited as open circles. Even though there are no experimental data for comparisons, the evaluated ratio values for ${ }^{237,238} \mathrm{~Np},{ }^{237} \mathrm{U},{ }^{242} \mathrm{Pu}$, and ${ }^{242} \mathrm{Cm}$ are also shown in all of these figures. All data are plotted as a function of principal actinide mass, generally without identifying the principal actinide charge. For example, the datum plotted for principal actinide mass $=230$ is the adjusted experimental value for the ${ }^{230} \mathrm{Th}$ sample; ${ }^{230} \mathrm{Th}$ is the only actinide of those in the present discussion having mass $=230$. For those principal actinide masses represented by more than one sample in either the evaluation or the experiment labels are appended to the plotted points to aid in differentiating the sources of the plotted points. For example, for mass $=238$, there are three evaluations (for ${ }^{238} \mathrm{U},{ }^{238} \mathrm{~Np}$, and ${ }^{238} \mathrm{Pu}$ ) and two experimental samples (for ${ }^{238} \mathrm{U}$ and ${ }^{238} \mathrm{Pu}$ ). In Fig. 11 for this mass, the open-circle points corresponding to the ${ }^{238} \mathrm{U}$ and ${ }^{238} \mathrm{Pu}$ evaluations are labelled, leaving the remaining (unlabelled) open-circle point as being for the ${ }^{238} \mathrm{~Np}$ evaluation; similarly the ${ }^{238} \mathrm{Pu}$ experimental datum for mass $=238$ is labelled so that the unlabelled experimental datum for mass $=$ 238 is for ${ }^{238} \mathrm{U}$. For mass $=244$ in Fig. 11 , the data for both of the two ${ }^{244} \mathrm{Cm}$ samples are labelled since two different experimental values were deduced from the data. We recognize that this manner of labelling is somewhat incomplete and leaves the reader the mental task of "completing the picture." In our defense, it was our desire to exhibit overall trends (rather than detailed comparisons) with these figures, and so to reduce the distraction from the main point we minimized the labelling.

These eight figures not only provide a visual comparison of the experimental results with the current state of knowledge, but provide an additional insight into the behavior of fissioning systems. As an example, in Fig. 11 one may observe a general behavior of the yield of ${ }^{91} \mathrm{Y}$ as one progresses through the fissioning systems from the lightest to the heaviest principal actinides. Indeed, different behaviors are observed, and these are discussed for each fission product.

\subsection{1 ${ }^{91} \mathrm{Y}$ Yield}

The experimental results reproduce reasonably well the trend of the evaluated data, but the experimental data also appear to be $-20 \%$ or so too small. One may consider several plausible explanations for this observation. The branching ratio for the observed gamma ray is small, $0.3 \%$ (as given in Table 2), and, despite the assigned ${ }^{4} 10 \%$ uncertainty, could be $\sim 20 \%$ smaller. Another plausible explanation seems less likely. This explanation has to do with the fact that the most-probable mass 91 isotope formed in the fissioning process is ${ }^{91} \mathrm{Kr}$, a noble gas. Although the half life of ${ }^{91} \mathrm{Kr}$ is quite short ( $\left.\sim 9 \mathrm{~s}\right)$, there may be the possibility of diffusion of the krypton through the thin vanadium walls. At this writing diffusion rates of krypton through thin metal foils are not well known, but one could conceive that some fraction of the ${ }^{91} \mathrm{Kr}$ could have escaped the vanadium cell, and the result would appear as observed in Fig. 11. 


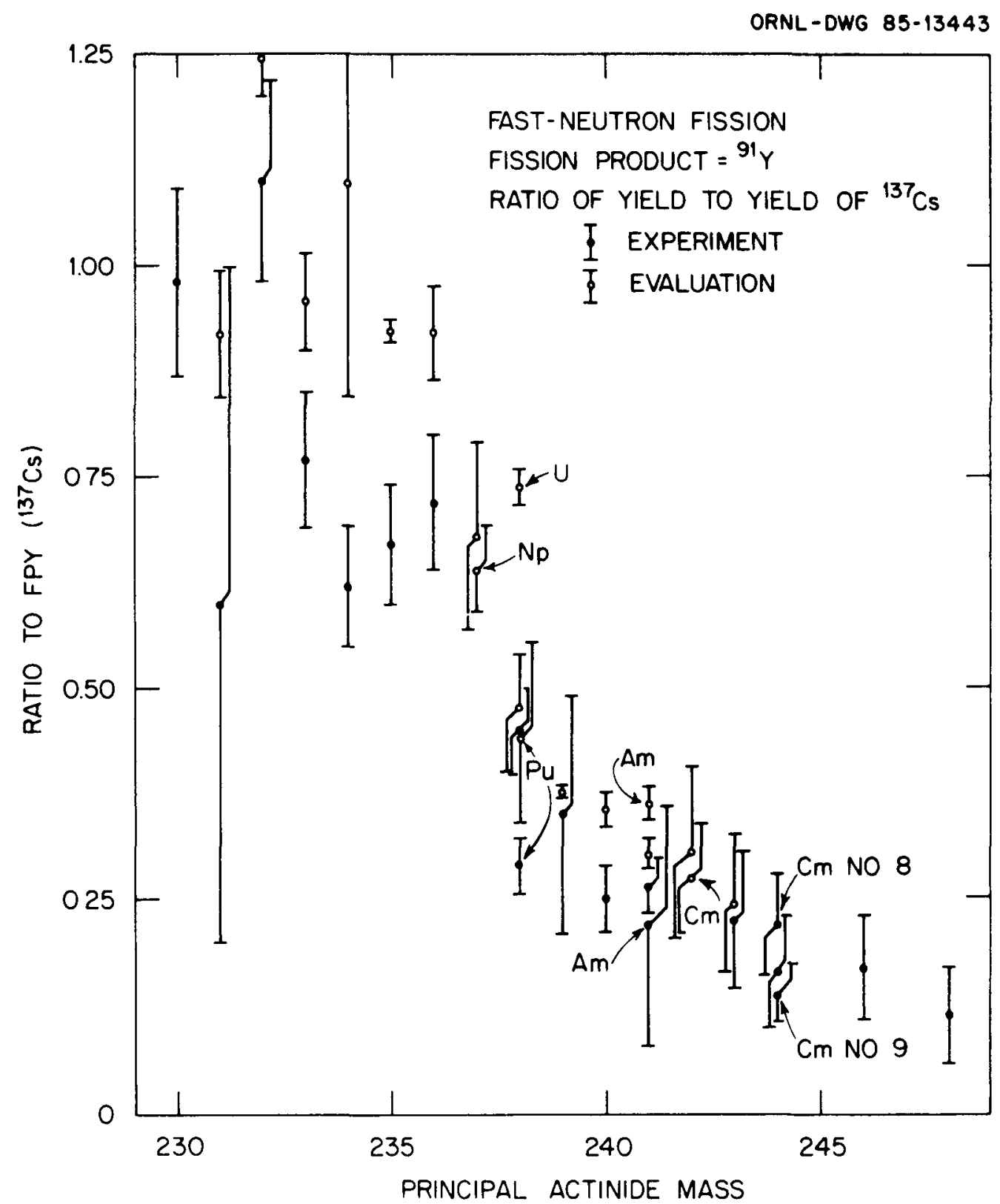

Fig. 11. Relative yields of ${ }^{91} \mathrm{Y}$ following fast-neutron fission of actinides between ${ }^{230} \mathrm{Th}$ and ${ }^{248} \mathrm{Cm}$. The present measurements are plotted as ratios to the experimental ${ }^{137} \mathrm{Cs}$ fission-product yields. As discussed in the text, the data appear to be about $-20 \%$ smaller than equivalent ratios deduced from the evaluation of Ref. 8; possibly the difference could be rectified by a $20 \%$ change in the very small branching ratio for the $1204-\mathrm{keV}$ gamma ray following decay of ${ }^{81} \mathrm{Y}$. 


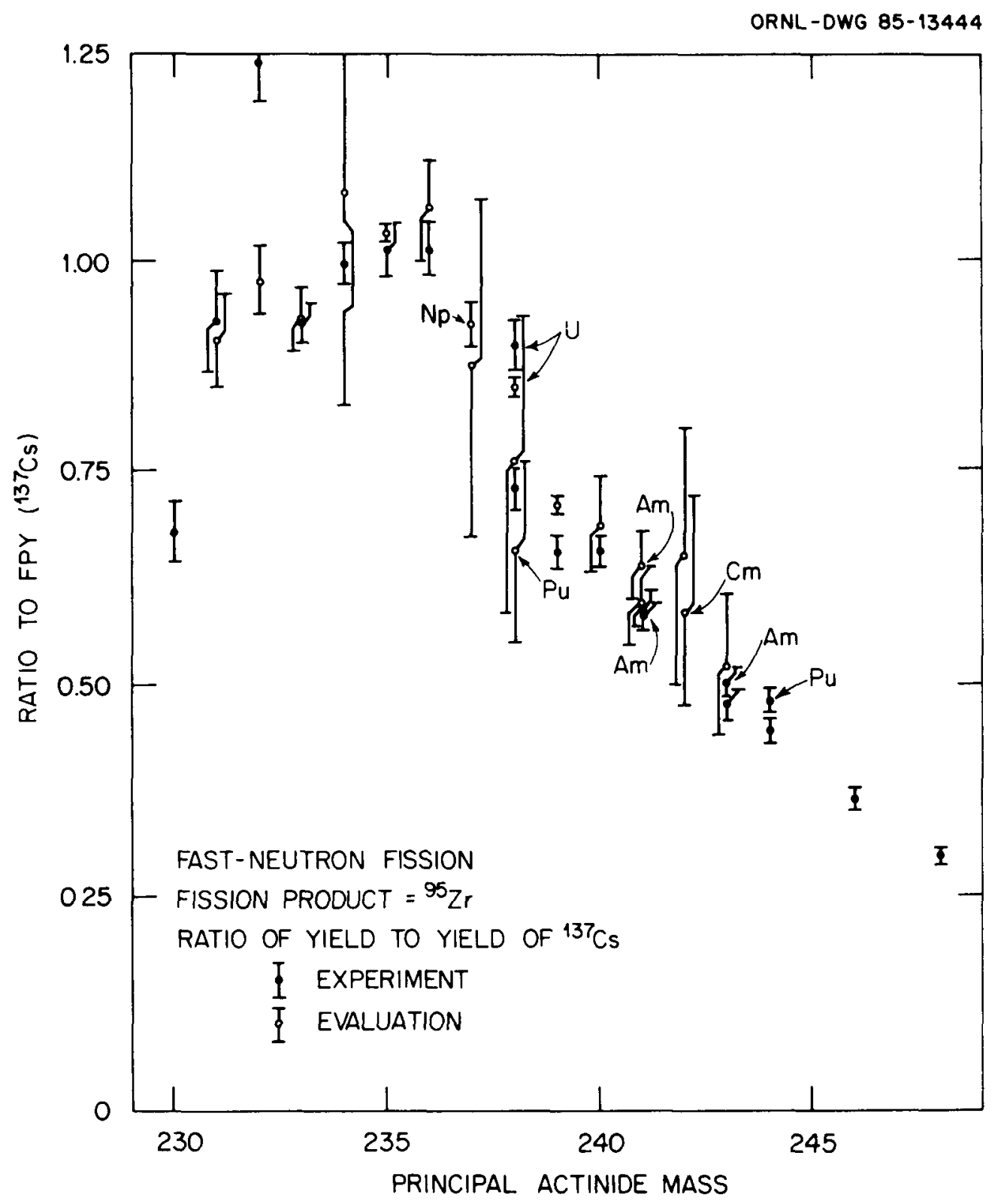

Fig. 12. Relative yields of ${ }^{95} \mathrm{Zr}$ following fast-neutron fission of actinides between ${ }^{230} \mathrm{Th}$ and ${ }^{248} \mathrm{Cm}$. The present measurements are plotted as ratios to the experimental ${ }^{137} \mathrm{Cs}$ fission-product yields. The data agree well with equivalent ratios deduced from the evaluation. 


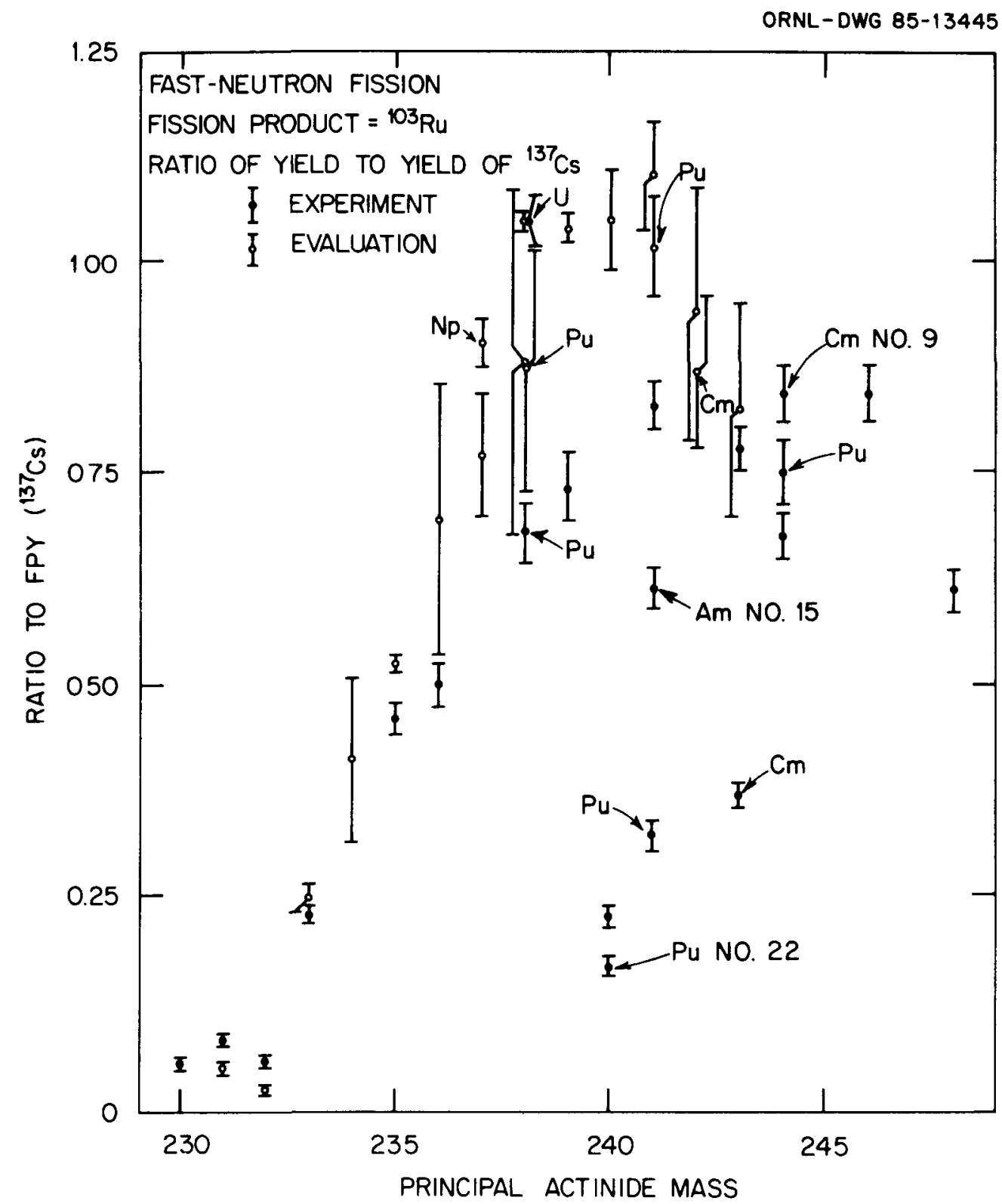

Fig. 13. Relative yields of ${ }^{103} \mathrm{Ru}$ following fast-neutron fission of actinides between ${ }^{230} \mathrm{Th}$ and ${ }^{248} \mathrm{Cm}$. The present measurements are plotted as ratios to the experimental ${ }^{137} \mathrm{Cs}$ fission-product yields. We are unable to explain the lack of agreement with some equivalent ratios deduced from the evaluation, particularly for ${ }^{240} \mathrm{Pu},{ }^{241} \mathrm{Pu},{ }^{241} \mathrm{Am}$ (sample \#15), and ${ }^{243} \mathrm{Cm}$. 


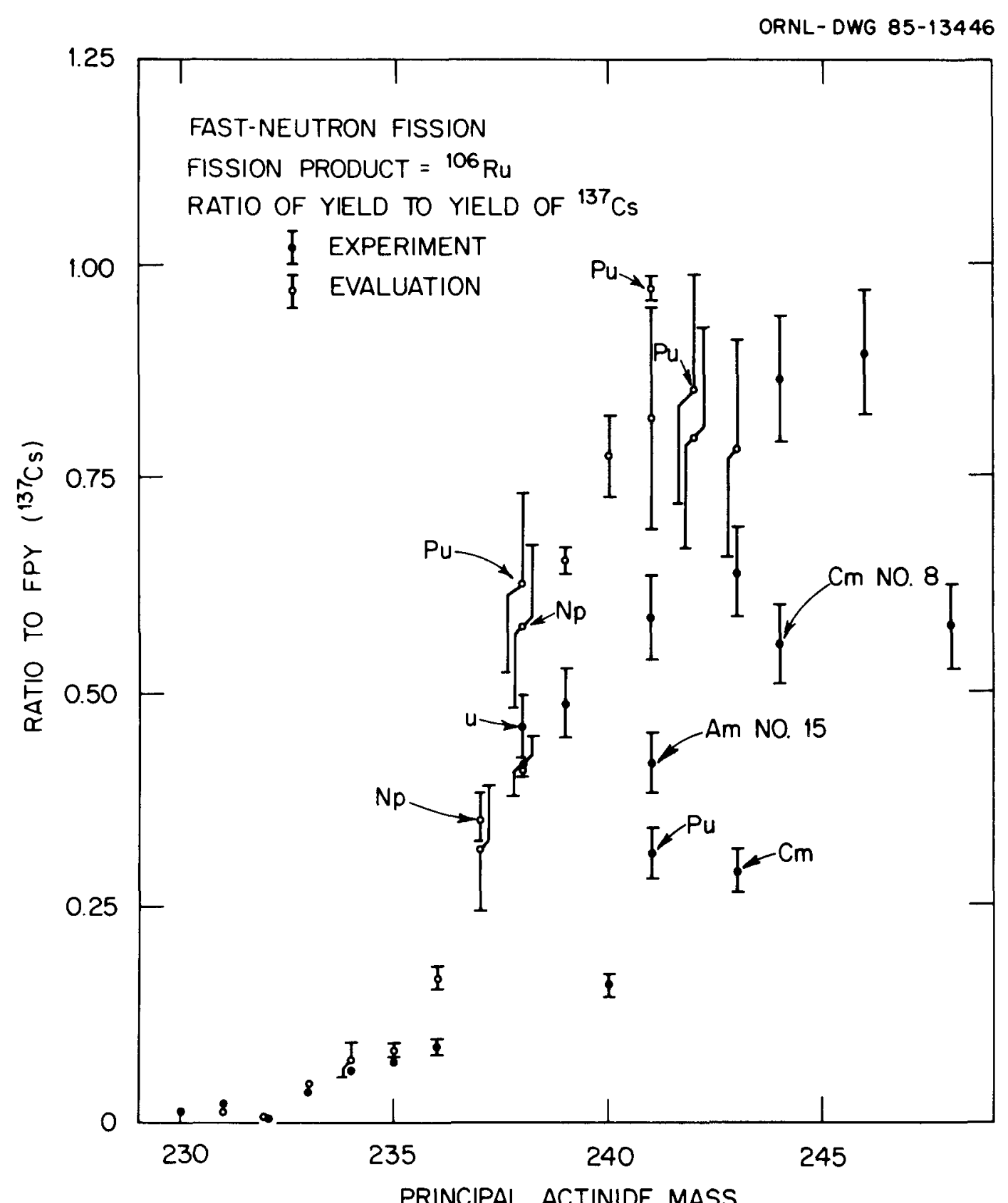

Fig. 14. Relative yields of ${ }^{106} \mathrm{Ru}-{ }^{106} \mathrm{Rh}$ following fast-neutron fission of actinides between ${ }^{230} \mathrm{Th}$ and ${ }^{248} \mathrm{Cm}$. The present measurements are plotted as ratios to the experimental ${ }^{137} \mathrm{Cs}$ fission-product yields. The unaccountable behavior observed in Fig. 13 for ${ }^{103} \mathrm{Ru}$ is observed in this figure also. 


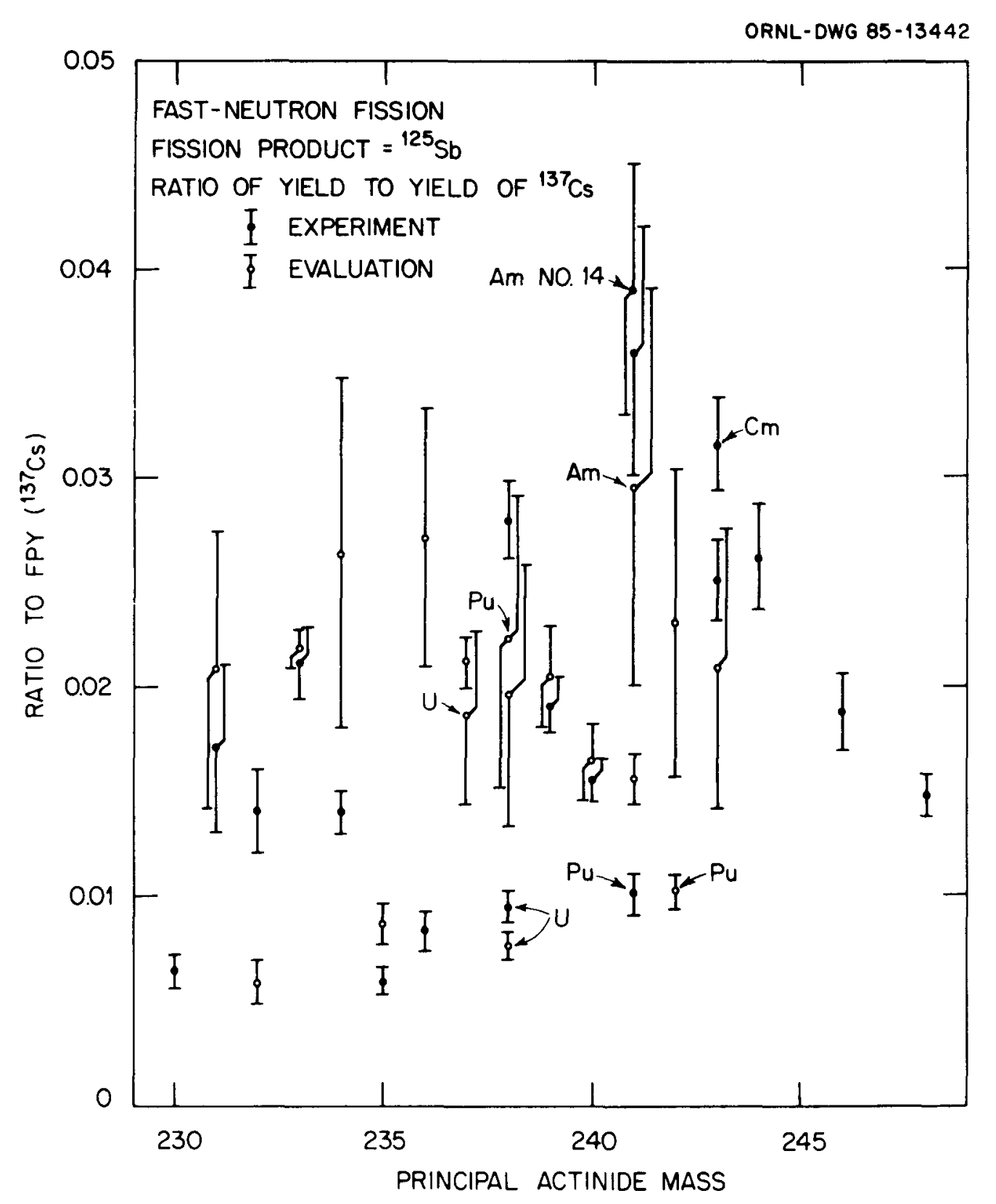

Fig. 15. Relative yields of ${ }^{125} \mathrm{Sb}$ following fast-neutron fission of actinides between ${ }^{230} \mathrm{Th}$ and ${ }^{248} \mathrm{Cm}$. The present measurements are plotted as ratios to the experimental ${ }^{137} \mathrm{Cs}$ fission-product yields. No distinct trend as a function of actinide mass is evident. 


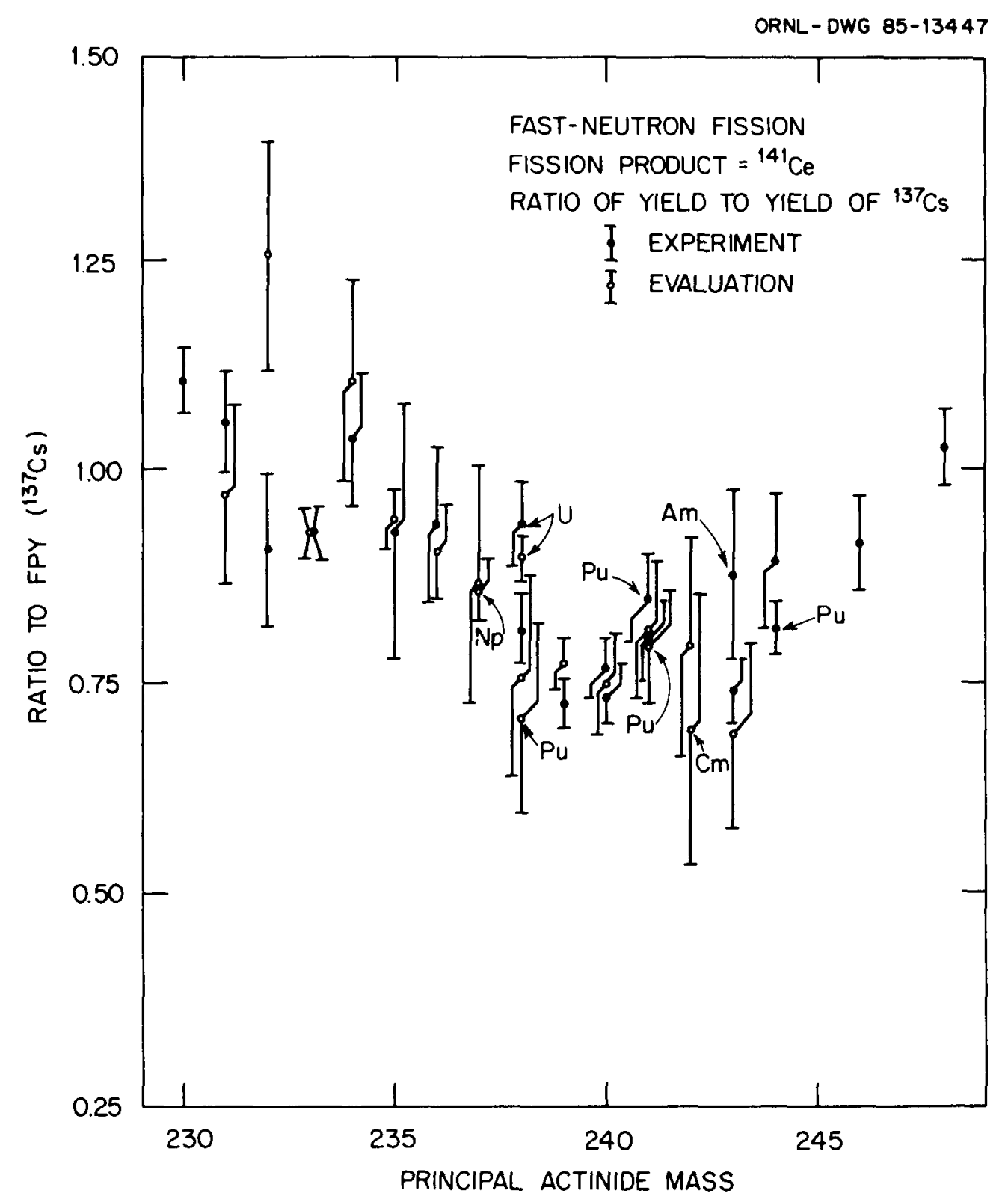

Fig. 16. Relative yields of ${ }^{141} \mathrm{Ce}$ following fast-neutron fission of actinides between ${ }^{230} \mathrm{Th}$ and ${ }^{248} \mathrm{Cm}$. The present measurements are plotted as ratios to the experimental ${ }^{137} \mathrm{Cs}$ fission-product yields. The experimental data agree quite well with equivalent ratios deduced from the evaluation. 


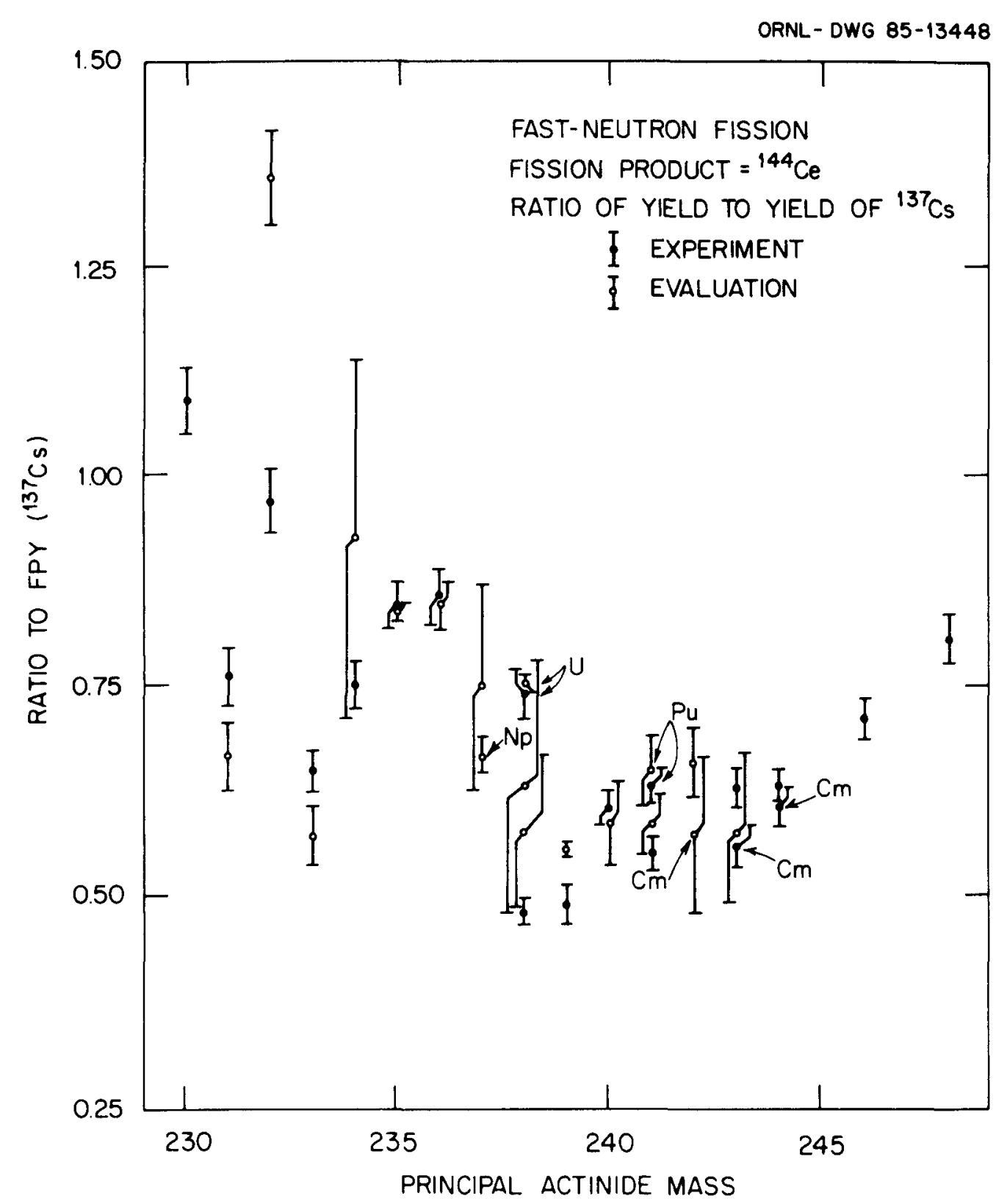

Fig. 17. Relative yields of ${ }^{144} \mathrm{Ce}-{ }^{144} \mathrm{Pr}$ following fast-neutron fission of actinides between ${ }^{230} \mathrm{Th}$ and ${ }^{248} \mathrm{Cm}$. The present measurements are plotted as ratios to the experimental ${ }^{137} \mathrm{Cs}$ fission-product yields. The experimental results agree reasonably well with equivalent ratios deduced from the evaluations for most of the principal actinides. 


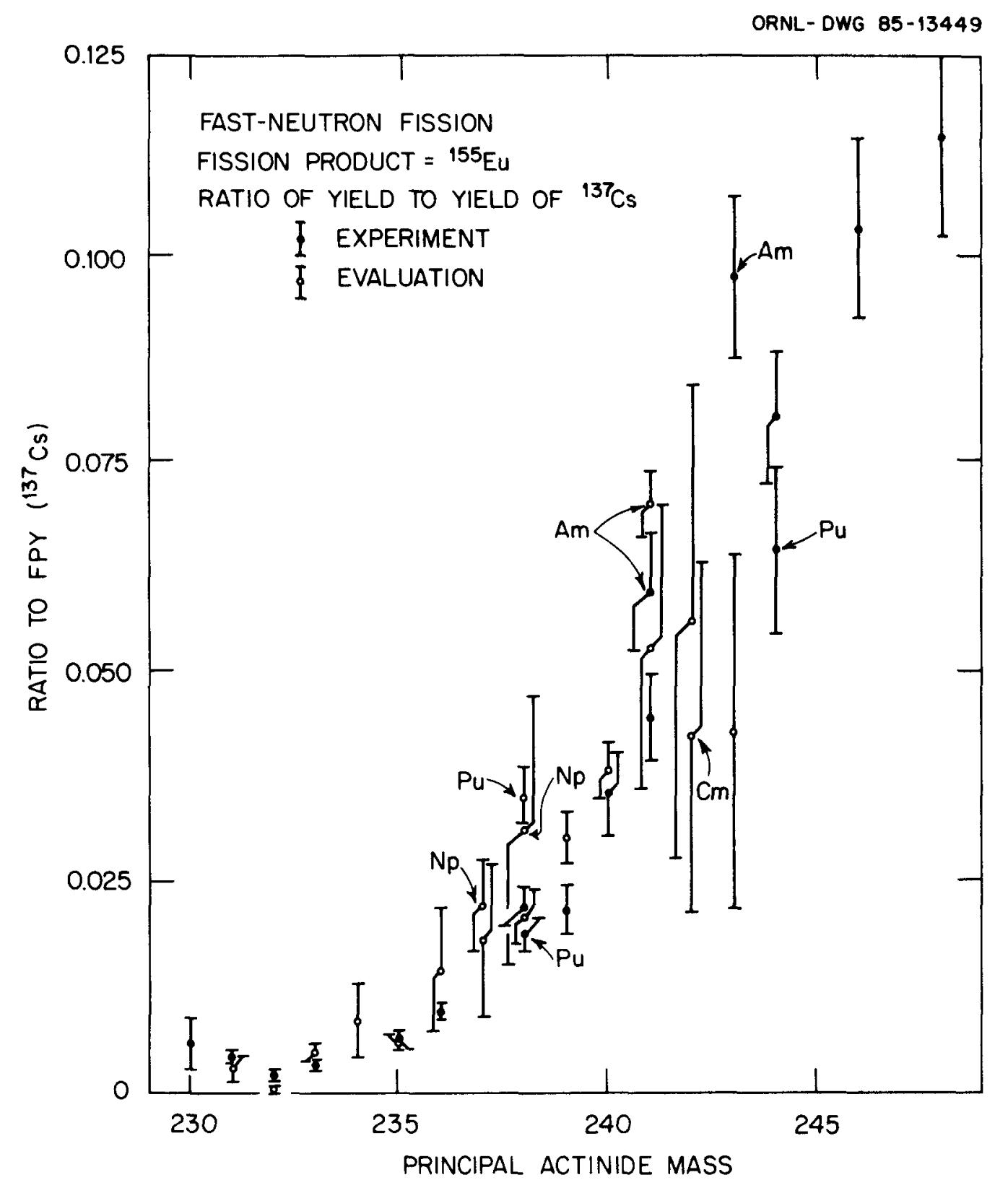

Fig. 18. Relative yields of ${ }^{155} \mathrm{Eu}$ following fast-neutron fission of actinides between ${ }^{230} \mathrm{Th}$ and ${ }^{248} \mathrm{Cm}$. The present measurements are plotted as ratios to the experimental ${ }^{137} \mathrm{Cs}$ fission-product yields. A definite trend as a function of actinide mass is observed. The agreement with equivalent ratios deduced from the evaluation is reasonable. 


\subsection{2 ${ }^{95} \mathrm{Zr}$ Yield}

The two primary decay gamma rays from decay of this fission product (see Table 2) are very pronounced in all of the spectra and provide an unambiguous identification of ${ }^{95} \mathrm{Zr}$, as well as a reliable determination of the yield of this radionuclide. As shown in Fig. 12, the experimental data agree quite well with the evaluated values, with only one rather substantial disagreement. The experimental value for fast-neutron fission of ${ }^{232} \mathrm{Th}$ is $-25 \%$ larger than the evaluated value. The $\sigma_{f}$ for ${ }^{232} \mathrm{Th}$ is comparatively very small, and the present experimental result may indicate a contribution from an unrelated ${ }^{94} \mathrm{Zr}(n, \gamma){ }^{95} \mathrm{Zr}$ reaction with "contaminant" elemental zirconium. If so, and if the amount of ${ }^{95} \mathrm{Zr}$ from this source, namely $\sim 20 \%$ of the observed yield, is indicative of the amount of ${ }^{95} \mathrm{Zr}$ from this source in the fission spectra for all of the other principal actinides, then such "contaminant" contributions to all the other measurements will be too small to require corrections. The good agreement of the experimental values with the evaluated values for the rest of the actinides provides a quantitative measure of the reliability of the data reduction and analyses detailed in previous sections.

\subsection{3 ${ }^{103}$ Ru Yield}

Identification of decay of this radionuclide depended on locating and evaluating a moderately sized peak in the raw data (see Fig. 6) only for the first set of experimental data since even for that set the cooling time from the end of the irradiation ( $\sim 300$ days, on the average) was already a factor of $\sim 7$ larger than the half life of ${ }^{103} \mathrm{Ru}$. Even so, for most of the spectra an apparently reliable determination of the yield of this fission product was obtained. However, as is readily observed in Fig. 13, there is an unsatisfactory lack of agreement of experimental values with evaluated values, especially for fast-neutron fission of ${ }^{240} \mathrm{Pu}$ and ${ }^{241} \mathrm{Pu}$. We are unable to explain the observed behavior as any kind of a physical phenomenon. Not only are there disagreements between evaluated values and experimental values, the experimental values for the three actinides having two samples $\left({ }^{240} \mathrm{Pu},{ }^{241} \mathrm{Am}\right.$, and ${ }^{244} \mathrm{Cm}$ ) also exhibit rather substantial differences between the results of the two samples for the same actinide. The spectral data and analyses were completely reviewed without locating an experimental error of the magnitude necessary to account for these observed discrepancies.

\subsection{4 ${ }^{106} \mathbf{R u}-{ }^{106} \mathrm{Rh}$ Yield}

For this case identification of the short-lived daughter, ${ }^{106} \mathrm{Rh}$, of the long-lived parent, ${ }^{106} \mathrm{Ru}$, is quite positive, and a reliable determination of the amount of this radionuclide in a given sample should be quite unambiguous. Indeed, analyses of the spectra obtained during November, 1984 confirm the yields obtained from the analyses of the spectra obtained during June and July, 1984. There is no indication that we had any unsuspected losses from the samples while they were in our possession. However, as exhibited in Fig. 14, the same lack of agreement among the experimental values with evaluated values is observed as was exhibited for ${ }^{103} \mathrm{Ru}$ in Fig. 13. Indeed, one may observe a substantial, if relative, correlation between ratio values for ${ }^{103} \mathrm{Ru}$ and those for ${ }^{106} \mathrm{Ru}-{ }^{106} \mathrm{Rh}$. This observation suggests, but most certainly does not dictate, that a loss of elemental ruthenium could have occurred during the chemical processing prior to preparation and packaging of our aliquots of these samples. Whatever the cause for the observed discrepancies, it seems reasonable to suggest that the results for ${ }^{103} \mathrm{Ru}$ and ${ }^{106} \mathrm{Ru}$ are not unequivocally reliable, including even those for the samples of uranium for which the experimental values appear to be in relatively good agreement with the evaluated values. 


\subsection{5 ${ }^{125}$ Sb Yield}

The strongest gamma ray observed in decay of this radionuclide is accidently nearly degenerate with a relatively weak gamma ray which occurs in decay of ${ }^{106} \mathrm{Rh}$, requiring that a correction be made for the ${ }^{106} \mathrm{Rh}$ decay contribution to that peak. Other ${ }^{125} \mathrm{Sb}$ gamma rays, however, provide adequate identification and quantitative determination for this fission product, which has a quite small yield for fast-neutron fission of all actinides studied. In addition, as exhibited by the evaluated ratios plotted in Fig. 15, there is no observable (at least visually) trend of fission-product yield vis-a-vis the principal actinide. Indeed, the agreement between experimental ratio values and evaluated ratio values is good for the samples having principal actinides of ${ }^{231} \mathrm{~Pa},{ }^{233} \mathrm{U},{ }^{239} \mathrm{Pu}$, and ${ }^{240} \mathrm{Pu}$, and within uncertainties for samples having principal actinides of ${ }^{238} \mathrm{Pu},{ }^{241} \mathrm{Am}$, and ${ }^{243} \mathrm{Am}$. For the ${ }^{232} \mathrm{Th}$ sample, more than half of the fissions are estimated to be due to fission of ${ }^{233} \mathrm{U}$. The ratio value plotted (at 0.014 ) is about that expected if for this sample $50 \%$ of the fissions were from ${ }^{232} \mathrm{Th}$ and the other $50 \%$ were from ${ }^{233} \mathrm{U}$. Similarly, the ratio value observed for the sample designated as ${ }^{236} \mathrm{U}$ appears to be too small, partly because $-60 \%$ of the fissions were due to the ${ }^{235} \mathrm{U}$ in the sample. Of some concern, however, is the rather poor agreement for the other samples, in particular those for the principal actinides of ${ }^{235} \mathrm{U},{ }^{238} \mathrm{U}$, and ${ }^{241} \mathrm{Pu}$. After complete review, we were unable to trace these discrepancies to errors in the experiment.

\subsection{6 ${ }^{141}$ Ce Yield}

Identification of decay of this radionuclide depenued on locating and evaluating a single, relatively small peak in the raw data (see Figs. 5 and 6) and only for the June and July, 1984 data set. The appropriate peak was observed in all LEPS spectra and in most of the $\mathrm{Ge}(\mathrm{Li})$ spectra. Results for this radionuclide required the largest correction for the irradiation history because of its short half life. Comparisons of experimental ratio values with evaluated values, as shown in Fig. 16, show excellent agreement; only for the sample of ${ }^{232} \mathrm{Th}$ is there disagreement, and this disagreement is at least partly accounted for by the ${ }^{233} \mathrm{U}$ contribution mentioned above.

\subsection{7 ${ }^{144} \mathrm{Ce}-{ }^{144} \mathrm{Pr}$ Yield}

For this case identification of the short-lived daughter was very positive. The agreement among the experimental ratio values with the evaluated values, as exhibited in Fig. 17, is not as good as observed for the ${ }^{141} \mathrm{Ce}$ data shown in Fig. 16. The apparent disagreement for ${ }^{232} \mathrm{Th}$ can be ameliorated to a large extent by the estimated ${ }^{233} \mathrm{U}$ contribution. However, the disagreements for the samples having principal actinides of ${ }^{233} \mathrm{U}$ and ${ }^{239} \mathrm{Pu}$ are a little difficult to understand, at least if they are due to errors in the experiment, particularly in view of the excellent agreement for samples having principal actinides of ${ }^{235} \mathrm{U},{ }^{238} \mathrm{U}$, and ${ }^{241} \mathrm{Pu}$.

\subsection{8 ${ }^{155}$ Eu Yield}

The two gamma rays observed in decay of this radionuclide are both rather small in energy, and often one was degenerate with a gamma ray or $\mathrm{x}$ ray following decay of the principal actinide in the sample. In addition, this fission product has a quite small yield for fast-neutron fission of the actinides studied, although as exhibited in Fig. 18 (unlike for ${ }^{125} \mathrm{Sb}$ ) there appears to be a rather definite trend toward larger yields for the heavier actinides. 
Apparently, what is being observed relates at least partly to a moderate broadening of the heavy-mass group and perhaps partly to a small overall shifting of this group toward heavier masses. An equally important observation, as exhibited in Fig. 18, is that the experimental ratio data are in reasonable agreement with evaluated ratio data, except for the ${ }^{238} \mathrm{Pu}$ and ${ }^{239} \mathrm{Pu}$ samples.

\subsubsection{Summary}

In summary, the experimental ratio values are in satisfactory agreement with evaluated ratios for ${ }^{95} \mathrm{Zr},{ }^{141} \mathrm{Ce},{ }^{144} \mathrm{Ce}-{ }^{144} \mathrm{Pr}$, and ${ }^{155} \mathrm{Eu}$, and perhaps in less satisfactory agreement with evaluated ratios for ${ }^{125} \mathrm{Sb}$. For those samples for which the experimental ratio values do not agree with the evaluated ratio values (at least to within combined assigned uncertainties), the experimental data were reviewed. For these five fission products the experimental data appear to be "correct" in the sense that we have not been able to find errors in the experiment.

The comparisons of experimental ratios with evaluated ratios for ${ }^{91} \mathrm{Y}$ indicate an apparent "constant" discrepancy of $-20 \%$ which could well be due to use (by us) of an incorrect branching ratio for the observation of the $1204-\mathrm{keV}$ gamma ray following decay of ${ }^{91} \mathrm{Y}$. And lastly, the experimental results for the two ruthenium radionuclides do not agree well with evaluated ratio values; we are unable to account for these discrepancies and so therefore do not know how to correct for them. We report results for these two radionuclides as we obtained them, however, with the recommendation that until we better understand all of the processes involved in sample preparation, the data, as reported in Table 7 for these fission products, should be treated with caution. Quite likely, some of the experimental data will be usable once the mechanisms leading to apparent disagreements with evaluated data are understood. In our opinion, the factors affecting the ${ }^{103} \mathrm{Ru}$ and ${ }^{106} \mathrm{Ru}-{ }^{106} \mathrm{Rh}$ measurements are peculiar only to the element ruthenium and should not impact either upon the other measurements nor upon their reliabilities.

\subsection{HEAVY-ELEMENT ACTINIDE YIELDS}

As mentioned above, peaks were observed in various gamma-ray spectra which could be assigned as detection of gamma rays following decay of radioisotopes in the mass region corresponding to the principal actinides being studied. Yields of these heavy elements were deduced from the spectra in units of the number of atoms, usually specified at the time of the end of the irradiation (EOI). However, the preanalysis calculations of Broadhead et al. ${ }^{2}$ gave results in units of mass at a time corresponding to EOI + 400 days (and, as mentioned above, for an irradiation of 90 FPD). In the discussions that follow, therefore, the measured amounts are given in units of mass (in grams) and at times specified in relation to the actual EOI.

\subsection{1 ${ }^{230}$ Th Sample}

Heavy elements definitely observed through their gamma-ray decay include ${ }^{226} \mathrm{Ra}$ (and daughters), ${ }^{228} \mathrm{Th}$ (and daughters) and ${ }^{233} \mathrm{~Pa}$. Analyses of the measurements provided yield data as follows: 


$$
\begin{array}{ll}
{ }^{226} \mathrm{Ra}: & (9.4 \pm 1.1) \times 10^{-9} \mathrm{~g} \text { at EOI }+435 \mathrm{~d} ; \\
{ }^{228} \mathrm{Th}: \quad & (1.25 \pm 0.15) \times 10^{-10} \mathrm{~g} \text { at EOI }+281 \mathrm{~d} \\
& (1.57 \pm 0.14) \times 10^{-10} \mathrm{~g} \text { at EOI }+435 \mathrm{~d} \\
& (1.90 \pm 0.13) \times 10^{-10} \mathrm{~g} \text { at EOI }+600 \mathrm{~d} \\
{ }^{233} \mathrm{~Pa}: \quad & (1.20 \pm 0.11) \times 10^{-8} \mathrm{~g} \text { at EOI. }
\end{array}
$$

These results should assist in determining our sample characteristics. In the first place, as already discussed above, the presence of ${ }^{226} \mathrm{Ra}$ was our first indication that the primary actinide of the sample was, in fact, ${ }^{230} \mathrm{Th}$ and not ${ }^{233} \mathrm{U}$. Indeed, the amount is larger than expected from the preanalysis calculations ${ }^{2}$ by about a factor of three (after allowing for our sample aliquot of $10 \%$ ).

The presence of ${ }^{233} \mathrm{~Pa}$ confirmed that our sample had the isotopic characteristics ${ }^{3}$ of the ${ }^{230} \mathrm{Th}$ sample. The total sample was expected to include $3.47 \times 10^{-4} \mathrm{~g}$ of ${ }^{232} \mathrm{Th}$ prior to the irradiation. One may compute the number of capture events of the type ${ }^{232} \mathrm{Th}(n, \gamma)$ using an effective $\sigma_{\gamma}=0.454 \mathrm{~b}$ taken from Table 20 of Ref. 2. For the total sample, then, $6.5 \times$ $10^{15}$ capture events were expected for a neutron fluence of 63 FPD at the ${ }^{230} \mathrm{Th}$ sample position, or $6.5 \times 10^{14}$ events for our aliquot, corresponding to $2.5 \times 10^{-7} \mathrm{~g}$ of ${ }^{233} \mathrm{U}$ after all of the ${ }^{233} \mathrm{~Pa}$ decayed. The half life of ${ }^{233} \mathrm{~Pa}$ is 26.95 days, ${ }^{4}$ and therefore accounting for the irradiation history of Table 9 requires multiplying the ${ }^{233} \mathrm{~Pa}$ yield at EOI by a factor of 5.9 to determine the total number of capture events for ${ }^{232} \mathrm{Th}$. Thus, in our sample the ${ }^{233} \mathrm{~Pa}$ measurement indicates a final ${ }^{233} \mathrm{U}$ mass of $\sim 7.1 \times 1^{n^{-8}} \mathrm{~g}$, or a factor of $\sim 3.5$ smaller than the amount of ${ }^{233} \mathrm{U}$ expected from neutron capture on the amount of the ${ }^{232} \mathrm{Th}$ supposedly in the sample on the basis of its original description. ${ }^{3}$ Recalling from Section 3.1.1 that the gamma-ray-assay direct measure of the ${ }^{230} \mathrm{Th}$ content of the sample was a factor of -3 smaller than expected, then the smaller yield of ${ }^{233} \mathrm{U}$ from the measurement approximately confirms our sample's thorium isotopic composition.

The ${ }^{228} \mathrm{Th}$ yields are listed as measured, since the yield of this radionuclide was evidently increasing with time. The amount of ${ }^{228} \mathrm{Th}$ is much too large and its growth is much too rapid to be due to decay of ${ }^{232} \mathrm{Th}$, and so must be indicative of the amount of ${ }^{232} \mathrm{U}$ in the sample. Thus, the experimental data provide a means to determine a fairly precise value of the amount of ${ }^{232} \mathrm{U}$ created during the irradiation even though the amount is too small to result in directly measurable yields of gamma rays due to decay of ${ }^{232} \mathrm{U}$.

\subsection{2 ${ }^{232}$ Th Sample}

For this sample large contributions to the observed spectra are due to detection of gamma rays from decay of ${ }^{233} \mathrm{~Pa}$. For this product of neutron capture in ${ }^{232} \mathrm{Th}$ we obtained

$$
{ }^{233} \mathrm{~Pa}:(2.78 \pm 0.18) \times 10^{-6} \mathrm{~g} \text { at } \mathrm{EOI},
$$

where the uncertainty includes an uncertainty of $\sim 6 \%$ associated with values of gamma-ray branching ratios ${ }^{4}$ as well as an uncertainty of $-2 \%$ related to the half life of ${ }^{233} \mathrm{~Pa}$ because the measurements were made some nine half lives after EOI. From the deduced ${ }^{233} \mathrm{~Pa}$ mass at EOI, one may determine that the total ${ }^{233} U$ mass produced by capture was $(1.64 \pm 0.11) \times$ $10^{-5} \mathrm{~g}$. This mass value may be compared with $1.82 \times 10^{-5} \mathrm{~g}$ estimated on the basis of our sample mass (from Table 2), its position in the fuel (to determine the total neutron fluence), and $\sigma_{\gamma}=0.454 \mathrm{~b}$. 


\subsection{3 ${ }^{231}$ Pa Sample}

By far the largest overall contributions to the observed spectra, in terms of disintegration rates, are from the gamma rays due to the decay of $1.91-\mathrm{yr}{ }^{228} \mathrm{Th}$ and its daughters. As was the situation for the ${ }^{230} \mathrm{Th}$ sample, the ${ }^{228} \mathrm{Th}$ decay that we observed must be due to ${ }^{232} \mathrm{U}$ decay, the ${ }^{232} \mathrm{U}$ being created as a result of neutron capture by ${ }^{231} \mathrm{~Pa}$ and the subsequent decay of 1.3-day ${ }^{232} \mathrm{~Pa}$ into ${ }^{232} \mathrm{U}$. Our measurements result in yield data as follows:

$$
\begin{aligned}
{ }^{228} \mathrm{Th}: \quad & (1.079 \pm 0.021) \times 10^{-7} \mathrm{~g} \text { at EOI }+321 \mathrm{~d} \\
& (1.316 \pm 0.026) \times 10^{-7} \mathrm{~g} \text { at EOI }+434 \mathrm{~d} \\
& (1.623 \pm 0.033) \times 10^{-7} \mathrm{~g} \text { at EOI }+683 \mathrm{~d}
\end{aligned}
$$

For comparison, the preanalysis calculations ${ }^{2}$ predicted $2.09 \times 10^{-6} \mathrm{~g}$ for the total sample at $\mathrm{EOI}+400 \mathrm{~d}$ for $90 \mathrm{FPD}$ irradiation, which would correspond to $-1.76 \times 10^{-7} \mathrm{~g}$ at EOI + $400 \mathrm{~d}$ for our $12 \%$ aliquot adjusted for 63 FPD irradiation.

\subsection{4 ${ }^{233} \mathrm{U}$ Sample}

Although the cross section for the ${ }^{233} \mathrm{U}(n, 2 n){ }^{232} \mathrm{U}$ reaction is smaller by almost four orders of magnitude than the cross section for neutron fission of ${ }^{233} \mathrm{U}$, the preanalysis calculations ${ }^{2}$ do include a prediction of the amount of ${ }^{232} U$ expected, and since the "signature" of ${ }^{232} U$ presence is ${ }^{228} \mathrm{Th}$ decay, we analyzed our data for evidence of decay of this radioisotope. A gamma ray having energy $E_{\gamma}=2614.5 \mathrm{keV}$ due to the decay of ${ }^{208} \mathrm{Tl}$, the lightest daughter in the radioactive chain initiated by the decay of ${ }^{228} \mathrm{Th}$, was observed at a detection rate of about twice the measured background rate for a peak at the gamma ray energy. Analysis of the data resulted in a yield for ${ }^{228} \mathrm{Th}$ as follows:

$$
{ }^{228} \mathrm{Th}:(2.4 \pm 0.8) \times 10^{-12} \mathrm{~g} \text { at EOI }+316 \mathrm{~d} .
$$

\subsection{5 ${ }^{234}$ U Sample}

There is a well-defined peak corresponding to the detection of a gamma ray having $E_{\gamma}=$ $2614.5 \mathrm{keV}$ in the first spectrum taken for this sample (i.e., during June 1984). Because of a different gain calibration, this gamma ray was too energetic to be observed in the spectrum obtained during November 1984. However, a second value was provided by a third measurement made during May 1985. The two yield results are

$$
\begin{aligned}
{ }^{228} \mathrm{Th}: & (1.7 \pm 0.2) \times 10^{-10} \mathrm{~g} \text { at EOI }+315 \mathrm{~d} \\
& (1.56 \pm 0.08) \times 10^{-10} \mathrm{~g} \text { at EOI }+624 \mathrm{~d}
\end{aligned}
$$

The source of this amount of ${ }^{228} \mathrm{Th}$ is not apparent either from the sample composition given in the report of Walker et al. ${ }^{3}$ or from the preanalysis calculations. ${ }^{2}$ It seems likely that prior to the irradiation the sample included perhaps $50 \%$ of the measured ${ }^{228} \mathrm{Th}$.

\subsection{6 ${ }^{236} U$ Sample}

Of the several heavy elements calculated to have yields $>10^{-6} \mathrm{~g}$ in the preirradiation analysis, ${ }^{2}$ the only one for which data were observed in the raw spectra that could be 
attributed to gamma radiation from its decay was ${ }^{238} \mathrm{Pu}$. A small peak corresponding to $E_{\gamma}$ $=43.39 \mathrm{keV}$ was observed; analysis of the data resulted in the following yield:

$$
{ }^{238} \mathrm{Pu}:(1.8 \pm 0.6) \times 10^{-7} \mathrm{~g} \text { at EOI. }
$$

This value is small, and close to the sensitivity of our measurements.

The data for ${ }^{236} \mathrm{U}$ were also studied for evidence of detection of gamma rays following decay of ${ }^{233} \mathrm{~Pa}$ as a "signature" of ${ }^{232} \mathrm{Th}$ in the sample, the ${ }^{232} \mathrm{Th}$ being a daughter of ${ }^{236} \mathrm{U}$. There was no indication at all of a peak in the raw data corresponding to $E_{\gamma}=311.8 \mathrm{keV}$; the sensitivity of the measurement for ${ }^{236} \mathrm{U}$ was such that we should have been able to quantitatively delineate a ${ }^{233} \mathrm{~Pa}$ mass of $1 \times 10^{-9} \mathrm{~g}$ at EOI, and probably to verify, at least qualitatively, the detection of decay of half that much ${ }^{233} \mathrm{~Pa}$. From these values one may estimate (see the discussions above on ${ }^{230} \mathrm{Th}$ and ${ }^{232} \mathrm{Th}$ ) an upper limit of $\sim 4 \times 10^{-7} \mathrm{~g}$ of ${ }^{232} \mathrm{Th}$ for our aliquot of this sample, or $-8 \times 10^{-6} \mathrm{~g}$ for the total sample.

\subsection{7 ${ }^{238}$ Pu Sample}

Spark source mass spectrometry (SSMS) of this sample resulted in the determination ${ }^{3}$ of $5000 \mathrm{ppm}$ by mass of ${ }^{232} \mathrm{Th}$. A peak corresponding to decay of ${ }^{233} \mathrm{~Pa}, E_{\gamma}=311.8 \mathrm{keV}$, was definitely observed. Analysis of the data provided the following yield:

$$
{ }^{233} \mathrm{~Pa}:(1.46 \pm 0.38) \times 10^{-9} \mathrm{~g} \text { at EOI. }
$$

As discussed above for the ${ }^{230} \mathrm{Th}$ and ${ }^{232} \mathrm{Th}$ samples, one may relate this measured yield to the mass of ${ }^{232} \mathrm{Th}$ in the sample. For the ${ }^{238} \mathrm{Pu}$ sample, the SSMS mass ratio implies an original mass of $\sim 17.5 \times 10^{-6} \mathrm{~g}$ of ${ }^{232} \mathrm{Th}$ in the sample, and from this mass one may compute $\sim 1.34 \times 10^{-7}$ of ${ }^{233} \mathrm{U}$ should have been created for the total sample, or $\sim 1.61 \times$ $10^{-8} \mathrm{~g}$ for our ostensible $12 \%$ aliquot. Correcting our measurement by 5.9 to account for the actual irradiation history yields a deduced value for ${ }^{233} U$ production of $(8.6 \pm 2.2) \times$ $10^{-9} \mathrm{~g}$, or a difference of $\sim 2$. Recall, however, the discussion of the amount of ${ }^{238} \mathrm{Pu}$ in the sample in section 3.1.3: our gamma-ray-assay mass for ${ }^{238} \mathrm{Pu}$ was smaller by $\sim 10$ than the expected ${ }^{238} \mathrm{Pu}$ mass. Hence, the measured ${ }^{233} \mathrm{~Pa}$ value implies that there is an inconsistency between ${ }^{232} \mathrm{Th}$ and ${ }^{238} \mathrm{Pu}$ masses in our sample which cannot be explained solely as due to an unlikely, but not prohibitive, error in determining our aliquot of the total sample.

\subsection{8 ${ }^{239}$ Pu Sample}

Preanalysis calculations ${ }^{2}$ indicate that there should be several heavy elements in this sample having yields which should be amenable to quantitative determination by careful analysis of our data. Of these elements, the gamma-ray-assay technique should be most sensitive to decay of ${ }^{241} \mathrm{Am}$, which is created by decay of ${ }^{241} \mathrm{Pu}$. The calculation of Ref. 2 results in a ${ }^{241} \mathrm{Am}$ yield of $2.05 \times 10^{-7} \mathrm{~g}$ at EOI +400 days. However, this yield was deduced for a neutron fluence of 90 FPD, and so a correction should be made for the actual neutron fluence of $63 \mathrm{FPD}$. In addition, the initial (preirradiation) amount of ${ }^{241} \mathrm{Pu}$ was given in Ref. 2 as $8.9 \times 10^{-7} \mathrm{~g}$ based on the isotopic analysis value of $0.011 \%$ of ${ }^{241} \mathrm{Pu}$ of the total elemental plutonium in the sample, as reported in Ref. 3. The isotopic analysis value, however, was performed in 1972, and so the actual percentage of ${ }^{241} \mathrm{Pu}$ in the sample was 
smaller than $0.011 \%$ at the beginning of the irradiation. It is a little difficult to determine precisely the amount of ${ }^{241} \mathrm{Am}$ that should have been created by decay of ${ }^{241} \mathrm{Pu}$ by a time given by EOI $+400 \mathrm{~d}$, but we have estimated $1.23 \times 10^{-7} \mathrm{~g}$ at EOI $+400 \mathrm{~d}$ as a reasonable value. How does this value compare with a yield deduced from the experimental data? The data do indicate evidence for detection of a gamma ray at about $E_{\gamma}=59.53 \mathrm{keV}$, the energy of the gamma ray having the largest yield in decay of ${ }^{241} \mathrm{Am}$, but the deduced energy of the observed peak is $\sim 0.15 \mathrm{keV}$ too small if our energy calibration is correct. In fact, the peak may be due to detection of more than one gamma ray. A better feeling for the problem can be gained from the experimental spectral data exhibited in Fig. 19; also included in this figure is the expected response to detection of 59.53-keV gamma rays following decay of $1.23 \times 10^{-8} \mathrm{~g}$ of ${ }^{241} \mathrm{Am}$, i.e., the mass corresponding to our aliquot of $10 \%$ of the total sample. Clearly, the ${ }^{241} \mathrm{Am}$ mass in our sample is less than $1.23 \times 10^{-8} \mathrm{~g}$. However, determining the mass of ${ }^{241} \mathrm{Am}$ from these data depends on deciding which of these data are really due to detection of a 59.53-keV gamma ray. Without any additional guidance we would treat the "peak" centered at $\sim 59.35 \mathrm{keV}$ as a doublet having a contribution from a $59.53-\mathrm{keV}$ gamma ray as the higher-energy response. The resulting yield of ${ }^{241} \mathrm{Am}$, assuming its decay is that responsible for the detected higher-energy gamma ray, would then be $\sim 2.5 \times 10^{-9} \mathrm{~g}$ for the measurement time EOI +446 days.

\subsection{9 ${ }^{240} \mathrm{Pu}$ Sample}

The only heavy element for which a quantitative yield could be determined from the experimental data was ${ }^{241} \mathrm{Am}$. Analysis of the measurements provided yield data as follows:

$$
\begin{array}{ll}
{ }^{241} \mathrm{Am} \text { for sample } 22: & (3.00 \pm 0.09) \times 10^{-7} \mathrm{~g} \text { at EOI }+326 \mathrm{~d} \\
{ }^{241} \mathrm{Am} \text { for sample } 21: & (2.20 \pm 0.06) \times 10^{-7} \mathrm{~g} \text { at EOI }+309 \mathrm{~d} \\
& (2.79 \pm 0.09) \times 10^{-7} \mathrm{~g} \text { at EOI }+446 \mathrm{~d}
\end{array}
$$

These values appear to be perhaps $\sim 10 \%$ smaller than estimated from preanalysis calculations of Broadhead et al. ${ }^{2}$ for the actual total neutron fluence of 63 FPD.

\subsubsection{0 ${ }^{241}$ Pu Sample}

Of the ten heavy elements other than ${ }^{241} \mathrm{Pu}$ for which Broadhead et al. ${ }^{2}$ computed yields $>1 \times 10^{-8} \mathrm{~g}$ at the end of the irradiation of this sample (for 90 FPD), we observed data ascribed to detection of gamma rays following decay of two of them. Analysis of the measurements provided yield data as follows:

$$
\begin{array}{ll}
{ }^{241} \mathrm{Am}: & (3.04 \pm 0.09) \times 10^{-5} \mathrm{~g} \text { at EOI }+329 \mathrm{~d} \\
& (3.13 \pm 0.09) \times 10^{-5} \mathrm{~g} \text { at EOI }+447 \mathrm{~d} \\
& (3.28 \pm 0.10) \times 10^{-5} \mathrm{~g} \text { at EOI }+622 \mathrm{~d} \\
{ }^{242} \mathrm{Cm}: \quad & (1.80 \pm 0.39) \times 10^{-7} \mathrm{~g} \text { at EOI }+329 \mathrm{~d}
\end{array}
$$

The mass values obtained for ${ }^{241} \mathrm{Am}$ are very consistent with the gamma-ray-assay deduced mass for our sample of $(5.03 \pm 0.19) \mathrm{g}$ of ${ }^{241} \mathrm{Pu}$ as reported in section 3.1.6. In addition, it is evident that there must have been $\sim 2.8 \times 10^{-5} \mathrm{~g}$ of ${ }^{241} \mathrm{Am}$ at the beginning of the irradiation in the sample, an amount which should contribute $\sim 5 \%$ of the total number of fissions created during the irradiation. Finally, the ${ }^{241} \mathrm{Am}$ mass results and ${ }^{241} \mathrm{Pu}$ mass results 


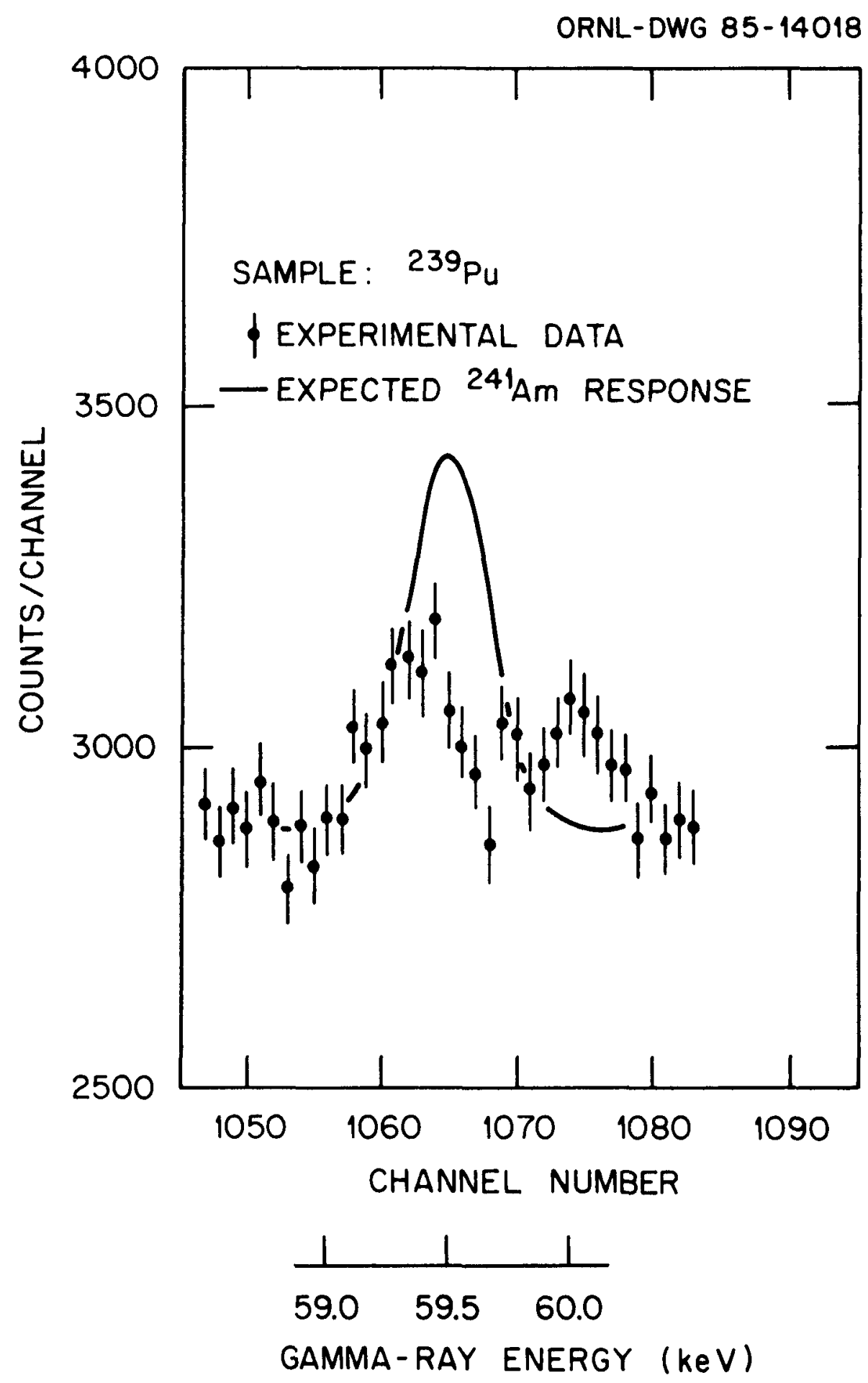

Fig. 19. Portion of the gamma-ray spectrum from a measurement of the ${ }^{239} \mathrm{Pu}$ sample using the intrinsic-Ge high-resolution detector. Also shown is an expected response for the $59.53-\mathrm{keV}$ gamma ray following decay of $12.3 \mathrm{ng}{ }^{241} \mathrm{Am}$ superimposed on a constant background of 2880 counts/channel. As discussed in the text, although a determination of the actual mass of ${ }^{241} \mathrm{Am}$ in this measurement would be highly uncertain, the predicted mass of $12.3 \mathrm{ng}$ of ${ }^{241} \mathrm{Am}$ is too large. 
are consistent with April 15, 1974 being the previous date of ${ }^{241}$ Am separation, as reported in Ref. 3. Interestingly, the $880 \mathrm{ppm}$ of ${ }^{237} \mathrm{~Np}$ in the sample ${ }^{3}$ can be computed from the estimated ${ }^{241} \mathrm{Am}$ in the sample at the beginning of the irradiation.

The amount of ${ }^{242} \mathrm{Cm}$ observed at EOI $+329 \mathrm{~d}$ may be converted to $1.33 \times 10^{-7} \mathrm{~g}$ of ${ }^{242} \mathrm{Cm}$ at $\mathrm{EOI}+400 \mathrm{~d}$. One may estimate, based on the preanalysis calculations ${ }^{2}$ for samples of principal actinide ${ }^{241} \mathrm{Am}$, that an irradiation of 63 FPD of an initial mass of $2.8 \times$ $10^{-5} \mathrm{~g}$ of ${ }^{241} \mathrm{Am}$ would result in production of ${ }^{242} \mathrm{Cm}$ of $1.47 \times 10^{-7} \mathrm{~g}$ at EOI $+400 \mathrm{~d}$. Detailed calculations of the type reported by Broadhead et al. ${ }^{2}$ are needed to refine this computation, e.g., calculations that would take into consideration the replenishment of ${ }^{241} \mathrm{Am}$ by the ${ }^{241} \mathrm{Pu}$ decay in the sample under discussion.

\subsubsection{1 ${ }^{244}$ Pu Sample}

Analysis of the gamma-ray data provided the following yield data:

$$
\begin{array}{ll}
{ }^{241} \mathrm{Am}: & (7.84 \pm 0.26) \times 10^{-7} \mathrm{~g} \text { at EOI }+327 \mathrm{~d} \\
{ }^{243} \mathrm{Am}: & (3.3 \pm 0.5) \times 10^{-7} \mathrm{~g} \text { at EOI }+327 \mathrm{~d} \\
{ }^{245} \mathrm{Cm}: & (1.73 \pm 0.21) \times 10^{-6} \mathrm{~g} \text { at EOI }
\end{array}
$$

Evidence of decay of other heavy isotopes, e.g., ${ }^{238} \mathrm{Pu}$ and ${ }^{242 \mathrm{~m}} \mathrm{Am}$, was sought in the data, but we could not unambiguously deduce yields for these radionuclides. Compared to the preirradiation analyses, ${ }^{2}$ the present sample (12\% aliquot) gamma-ray-assay results appear to be in good agreement with the calculated results for ${ }^{243} \mathrm{Am}$ and ${ }^{245} \mathrm{Cm}$ and to be somewhat smaller than the calculated results for ${ }^{241} \mathrm{Am}$. (The gamma-ray branching ratios for ${ }^{245} \mathrm{Cm}$ were taken from Ref. 9.)

\subsubsection{2 ${ }^{241}$ Am Sample}

Analysis of the data obtained for sample \#14 provided yield data as follows:

$$
\begin{aligned}
{ }^{242 m} \mathrm{Am}: & (8.15 \pm 0.24) \times 10^{-8} \mathrm{~g} \text { at EOI; } \\
{ }^{242} \mathrm{Cm}: & (7.2 \pm 1.0) \times 10^{-8} \mathrm{~g} \text { at EOI }+292 \mathrm{~d} \\
{ }^{243} \mathrm{Am}: & (1.9 \pm 0.6) \times 10^{-9} \mathrm{~g} \text { at EOI } \\
{ }^{243} \mathrm{Cm}: & (1.56 \pm 0.07) \times 10^{-9} \mathrm{~g} \text { at EOI }
\end{aligned}
$$

These mass values are within $-30 \%$ agreement with estimates based upon the preirradiation analyses. ${ }^{2}$ The yield for the ${ }^{242 \mathrm{~m}} \mathrm{Am}\left(T_{1 / 2}=152 \mathrm{yr}\right)$ was deduced from detected gamma rays assigned as decay of the $16-\mathrm{hr}$ daughter ${ }^{242} \mathrm{Am}$. The branching ratio of the $48.6-\mathrm{keV}$ gamma ray following decay of ${ }^{242 \mathrm{~m}} \mathrm{Am}$ is not known; however, one may estimate the total internal conversion coefficient to be $\sim 7.4 \times 10^{5}$ from the tables of Rosel et al. ${ }^{10}$ assuming a pure E4 multipolarity. A peak corresponding to $E_{\gamma}=48.6 \mathrm{keV}$ observed in the data has the correct energy for the ${ }^{242 \mathrm{~m}} \mathrm{Am}$ decay transition, but the extracted yield appears to be several orders of magnitude too large for such assignment, if the total internal conversion coefficient is at least as large as the above estimate.

\subsubsection{3 ${ }^{243}$ Am Sample}

None of the masses computed for heavy actinides other than ${ }^{243} \mathrm{Am}$ in the preirradiation analyses ${ }^{2}$ indicated a sufficient mass such that decay gamma radiation would be detected and 
properly identified. For example, the $E_{\gamma}=59.53 \mathrm{keV}$ gamma ray following decay of ${ }^{241} \mathrm{Am}$ is one of the easiest transitions to identify and quantify, but the estimated yield for this gamma ray was just at the edge of the system sensitivity. Indeed, for this sample (as for the sample of ${ }^{243} \mathrm{Cm}$ ), detection of the gamma rays from decay of the principal actinide dominated the spectral distributions that were measured.

\subsubsection{4 ${ }^{244} \mathrm{Cm}$ Sample}

Analysis of the gamma-ray data provided the following yield data:

$$
\begin{array}{ll}
{ }^{241} \mathrm{Am} \text { for sample \#8: } & (6.0 \pm 1.2) \times 10^{-9} \mathrm{~g} \text { at EOI; } \\
{ }^{241} \mathrm{Am} \text { for sample \#9: } & (3.2 \pm 0.2) \times 10^{-8} \mathrm{~g} \text { at EOI; } \\
{ }^{243} \mathrm{Am} \text { for sample \#8: } & (5.8 \pm 0.6) \times 10^{-7} \mathrm{~g} \text { at EOI; } \\
{ }^{243} \mathrm{Am} \text { for sample \#9: } & (2.77 \pm 0.15) \times 10^{-6} \mathrm{~g} \text { at EOI; } \\
{ }^{245} \mathrm{Cm} \text { for sample \#8: } & (9.2 \pm 0.7) \times 10^{-7} \mathrm{~g} \text { at EOI; } \\
{ }^{245} \mathrm{Cm} \text { for sample \#9: } & (4.05 \pm 0.29) \times 10^{-6} \mathrm{~g} \text { at EOI. }
\end{array}
$$

The measured yields for ${ }^{245} \mathrm{Cm}$ for the two samples appear to be consistent with expectations based upon the gamma-ray assay of the ${ }^{244} \mathrm{Cm}$ mass determinations for these two samples plus estimates of the capture reaction ${ }^{244} \mathrm{Cm}(n, \gamma){ }^{245} \mathrm{Cm}$ yields for 63 FPD of neutron fluence. The yields deduced for ${ }^{241} \mathrm{Am}$ appear moderately larger than the estimated yields for ${ }^{241} \mathrm{Am}$ obtained by first-order scaling of the preirradiation analyses of Ref. 2 . The observation of ${ }^{243} \mathrm{Am}$ was somewhat of a surprise. However, as mentioned above, gamma rays from the ${ }^{243} \mathrm{Am}$ decay and from the ${ }^{243} \mathrm{Cm}$ decay result in quite similar spectra. Indeed, a portion of the observed data must have been due to detection of gamma rays from decay of ${ }^{243} \mathrm{Cm}$, but the statistical uncertainties of the spectral data were rather poor, and so we were not able to extract yields for ${ }^{243} \mathrm{Cm}$ with a satisfactory reliability from the data.

\subsubsection{5 ${ }^{246} \mathrm{Cm}$ Sample}

Analysis of the gamma-ray data provided the following yield data (at EOI unless otherwise noted):

$$
\begin{aligned}
{ }^{241} \mathrm{Am}: & (9.5 \pm 0.9) \times 10^{-9} \mathrm{~g} ; \\
{ }^{243} \mathrm{Am}: & (1.55 \pm 0.16) \times 10^{-7} \mathrm{~g} \\
{ }^{243} \mathrm{Cm}: \quad & (1.31 \pm 0.09) \times 10^{-8} \mathrm{~g} \\
{ }^{244} \mathrm{Cm}: \quad & (3.0 \pm 0.3) \times 10^{-5} \mathrm{~g} \\
{ }^{245} \mathrm{Cm:}: & (8.2 \pm 2.2) \times 10^{-7} \mathrm{~g} ; \\
{ }^{249} \mathrm{Cf}: \quad & (1.10 \pm 0.06) \times 10^{-7} \mathrm{~g} \text { at EOI }+299 \mathrm{~d} \\
& (1.15 \pm 0.05) \times 10^{-7} \mathrm{~g} \text { at EOI }+439 \mathrm{~d}
\end{aligned}
$$

These results appear to be consistent with the results of the preirradiation analyses of Ref. 2 . Direct ratio estimates of yields of these isotopes, as we have done above, are somewhat less reliable for this principal actinide because of the comparatively substantial preirradiation abundances of the other curium isotopes in the sample. A complete calculation of the type reported by Broadhead $e t$ al. ${ }^{2}$ will be required in this case. 


\subsubsection{6 ${ }^{248} \mathrm{Cm}$ Sample}

Analysis of the gamma-ray data provided the following yield data for this heavy isotope:

$$
\begin{array}{ll}
{ }^{249} \mathrm{Cf}: & (6.47 \pm 0.29) \times 10^{-7} \mathrm{~g} \text { at EOI }+299 \mathrm{~d} \\
& (7.89 \pm 0.35) \times 10^{-7} \mathrm{~g} \text { at EOI }+437 \mathrm{~d} .
\end{array}
$$

These results appear to be consistent with the results of the preirradiation analysis of Ref. 2 after adjusting for a 63-FPD irradiation.

\subsubsection{Summary}

In concluding this discussion of yield measurements of the nonprincipal actinides, it should be mentioned that the absence of a reported yield should not be construed as an absence of possible detection of gamma radiation corresponding to decay of the radioisotope in question, nor even that the possible yield of said radioisotope is very small. We reported herein on those measurements for which identification with the reported actinide appeared to be reliable and unambiguous. In addition, as mentioned above, isotopes having long half lives $\left(>10^{5} \mathrm{y}\right.$ for certain) were simply not observed in the present series of measurements. So, many of the calculated yields given in the preirradiation analysis ${ }^{2}$ could not be tested. However, a number of data have been presented, and they should provide testing of future computational methods.

\section{SUMMARY AND CONCLUSIONS}

The purpose of this experiment was to provide information on yields of radioisotopes, principally fission products, created by an extended "fast-neutron" irradiation of a fuel pin containing 26 separate samples representing 21 different fissile actinides that either are found in the fuel of a fast reactor, or could be found if some alternate nuclear fuel cycle were to be utilized. Indeed, although many fission-product yields for fast-neutron fission have already been reported and subjected to evaluation, 8 there were six principal sample actinides included in this experiment for which such data have not previously been available. In addition, the present experiment was expected to provide additional information on all the measured yields by virtue of the simultaneous irradiation of all of the samples.

The results of the data analyses for fission-product yields are illustrated in Figs. 11 to 18 . In particular for ${ }^{95} \mathrm{Zr},{ }^{141} \mathrm{Ce},{ }^{144} \mathrm{Ce}-{ }^{144} \mathrm{Pr}$, and ${ }^{155} \mathrm{Eu}$, definite trends are observed for yields of these fission products as functions of the actinide sample being studied.

The gamma-ray-assay data also provided checks on several aspects of the experiment, in particular the masses of the aliquots of the samples we studied. In addition, some of the heavy actinides produced by capture reactions were amenable to quantitative determinations from study of the present data. Clearly, such results, while necessarily incomplete overall, do provide a more comprehensive picture of the nuclear processes which were induced by the total neutron irradiation. 
A second fuel pin, very similar in content to the pin whose elements were studied and reported upon in this report, and subjected to a similar 63 FPD irradiation, is awaiting further study. In addition to possibly shedding fresh light on discrepancies reported herein, controlled measurements could produce absolute fission-product yield data for ${ }^{137} \mathrm{Cs}$ and place all of the yield data on an absolute basis.

Although the present measurements did provide some results difficult to understand within the overall framework of the experiment (and these problems were discussed in sufficient detail to provide some basis for their accomodation in some future experiment and/or analysis), the measurements also yielded a substantial wealth of data for comparisons with detailed calculations. We have tried to present and discuss the data in such a way as to facilitate and guide such calculations and comparisons. One hopes, of course, that detailed and rigorous calculations will agree well with the data, for in such case a good comparison tends to support the validity of the calculational procedures as well as enhance credibility of the experimental measurements. From the experimental viewpoint, however, in the event of any unsatisfactory agreement with calculation, we can report only that the history of the experiment reported herein was completely reviewed, and we believe that the results are correctly presented as obtained. Sic passim.

\section{ACKNOWLEDGEMENTS}

We express our appreciation to D. A. Costanzo for our sample fabrications and to T. R. England for providing an up-to-date (and easy-to-read) listing of the yields evaluation. We thank B. L. Broadhead for enlightening discussions regarding calculational procedures and results and for a careful reading of several drafts of this report. In addition, we appreciate the efforts of many persons too numerous to mention for their work on sample preparations and irradiations. 


\section{$57 / 58$}

\section{REFERENCES}

1. T. C. Quinby, H. L. Adair, E. H. Kobisk, D. W. Ramey, J. A. Setaro, J. L. Botts, J. H. Cooper, R. L. Walker, J. E. Bigelow, J. R. Gibson, W. T. Martin, R. G. Pope, and S. Raman, Preparation of Actinide Specimens for the US/UK Joint Experiment in the Dounreay Prototype Fast Reactor, ORNL-5858, Oak Ridge National Laboratory, May 1982.

2. B. L. Broadhead, N. B. Gove, and S. Raman, Preanalysis Calculations of the US/UK Joint Experiment in the Dounreay Prototype Fast Reactor, ORNL-6058, Oak Ridge National Laboratory, May 1984.

3. R. L. Walker, J. L. Botts, J. H. Cooper, H. L. Adair, J. E. Bigelow, and S. Raman, Characterization of Actinide Physics Specimens for the US/UK Joint Experiment in the Dounreay Prototype Fast Reactor, ORNL-5986, Oak Ridge National Laboratory, October 1983.

4. C. M. Lederer, V. S. Shirley, E. Browne, J. M. Dairiki, R. E. Doebler, A. A. ShihabEldin, L. J. Jardine, J. K. Tuli, and A. B. Buyrn, Table of Isotopes, John Wiley and Sons, Inc., New York, 1978, Seventh Edition, 1978.

5. E. Storm and H. I. Israel, Nucl. Data Tables A7, 565 (1970).

6. J. K. Dickens, TPASS, A Gamma-Ray Spectrum Analysis and Isotope Identification Computer Code, ORNL-5732, Oak Ridge National Laboratory, March 1981.

7. TPASGAM, Radioactive Decay Library of Gamma-Ray Energies, Branching Ratios, and Cross Sections, RSIC Data Library Collection Report No. DLC-88 (1982) of the Oak Ridge National Laboratory.

8. B. F. Rider, Compilation of Fission Product Yields, NEDO-12154-3(C), ENDF-322, General Electric Company, 1981; an updated and unpublished listing was obtained from T. R. England (private communication, 1985).

9. J. K. Dickens and J. W. McConnell, Phys. Rev. C22, 1344 (1980).

10. F. Rosel, H. M. Fries, K. Alder, and H. C. Pauli, At. Data and Nucl. Data Tables 21, 292 (1978). 


\section{,} ,

-

.

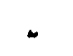




\section{INTERNAL DISTRIBUTION}

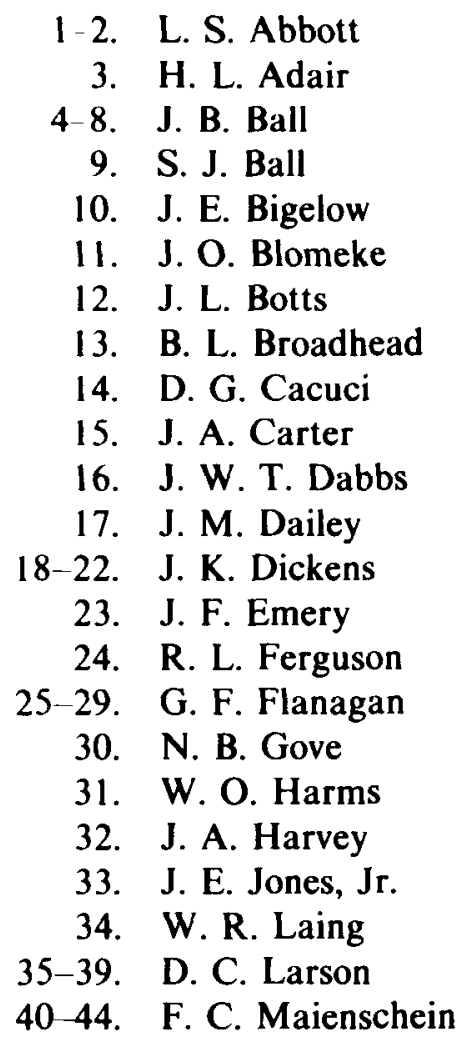
45. F. R. Mynatt
46. J. V. Pace
47-51. R. W. Peelle
52-56. F. G. Perey
57. H. Postma
58. T. C. Quinby
59-63. S. Raman
64. W. D. Schults
65. P. H. Stelson
66. D. B. Trauger
67. R. L. Walker
68. M. K. Wilkinson
69. A. Zucker
70. P. W. Dickson, Jr. (Consultant)
71. G. H. Golub (Consultant)
72. R. M. Haralick (Consultant)
73. D. Steiner (Consultant)
74-75. Central Research Library
76. Y-12 Document Ref. Section
77-78. Laboratory Records Department
79. Laboratory Records, ORNL-RC
80. ORNL Patent Office
81-83. EPMD Reports Office

\section{EXTERNAL DISTRIBUTION}

84. T. R. England, Los Alamos National Laboratory, P.O. Box 1663, Los Alamos, NM 87545

85. S. L. Whetstone, Division of Nuclear Sciences, Office of Basic Energy Sciences, U.S. DOE, Washington, DC 20545

86-90. E. T. Weber, Manager, Core Technology and Safety, Hanford Engineering Development Laboratory, P.O. Box 1970, Richland, WA 99352

91-95. K. M. Swanson, Dounreay Nuclear Power Development Establishment, Thurso, Caithness, Scotland, UK

96. M. L. Williams, Nuclear Science Center, Louisian State University, Baton Rouge, LA 70803

97. R. J. Neuhold, Division of Advanced Technology Department, Office of Technology Support Programs, Washington, DC 20545.

98. P. B. Hemmig, Division of Advanced Technology Department, Office of Technology Support Programs, Washington, DC 20545.

99. Office of Assistant Manager for Energy Research and Development, DOE, ORO

100. Director, Nuclear Research and Development Division, DOE-ORO

101-212. For distribution as shown in TID-4500, Distribution Category UC-79d - LMFBR Physics; Base Technology. 